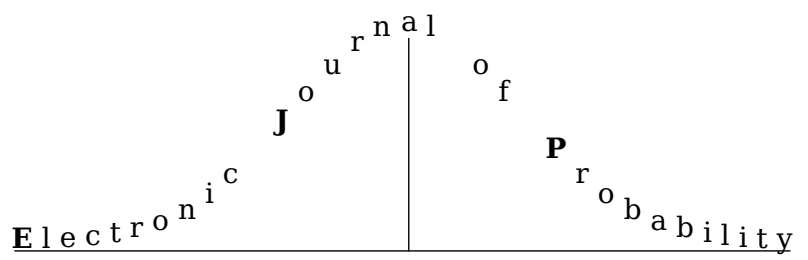

Electron. J. Probab. 26 (2021), article no. 152, 1-72.

ISSN: 1083-6489 https://doi.org/10.1214/21-EJP721

\title{
Estimation of local anisotropy based on level sets
}

\author{
Corinne Berzin*
}

\begin{abstract}
Consider an affine field $X: \mathbb{R}^{2} \rightarrow \mathbb{R}$, that is a process equal in law to $Z(A . t)$, where $Z$ is isotropic and $A: \mathbb{R}^{2} \rightarrow \mathbb{R}^{2}$ is a linear self-adjoint transformation. The field $X$ and transformation $A$ will be supposed to be respectively Gaussian and definite positive. Denote $0<\lambda:=\frac{\lambda_{2}}{\lambda_{1}} \leqslant 1$ the ratio of the eigenvalues of $A$, let $\lambda_{1}, \lambda_{2}$ with $\lambda_{2} \leqslant \lambda_{1}$. This paper is aimed at testing the null hypothesis " $X$ is isotropic" versus the alternative " $X$ is affine". Roughly speaking, this amounts to testing " $\lambda=1$ " versus " $\lambda<1$ ". By setting level $u$ in $\mathbb{R}$, this is implemented by the partial observations of process $X$ through some particular level functionals viewed over a square $T_{n}$, which grows to $\mathbb{R}^{2}$. This leads us to provide estimators for the affinity parameters that are shown to be almost surely consistent. Their asymptotic normality results provide confidence intervals for parameters.

This paper offered an important opportunity to study general level functionals near the level $u$ and for a fixed bounded rectangle $T$ of $\mathbb{R}^{2}$, part of the difficulties arises from the fact that the topology of level set $\mathrm{C}_{T, X}(u)=\{t \in T: X(t)=u\}$ can be irregular, even if the trajectories of $X$ are regular. A significant part of the paper is dedicated to show the $L^{2}$-continuity in the level $u$ of these general functionals.
\end{abstract}

Keywords: affine processes; isotropic processes; level sets; Rice formulas for random fields; test of isotropy; Gaussian fields.

MSC2020 subject classifications: Primary 62G10; 53C65; 62F12; 60G60, Secondary 60G10; $60 \mathrm{G} 15$.

Submitted to EJP on January 3, 2018, final version accepted on October 25, 2021.

Supersedes HAL: hal -01676491.

\section{Introduction}

The aim of the present paper is to test the null hypothesis that a given real Gaussian process $X$ indexed in $\mathbb{R}^{2}$ and living in the class of affine processes is isotropic. Recall that a process $\left\{X(t), t \in \mathbb{R}^{2}\right\}$ is said to be an affine process when it is equal in law to $\left\{Z(A . t), t \in \mathbb{R}^{2}\right\}$, where $Z$ is isotropic (see definition in Section 2.1, page 15) and $A$ : $\mathbb{R}^{2} \rightarrow \mathbb{R}^{2}$ is a linear self-adjoint matrix. We assume that $X$ is partially observed through

\footnotetext{
${ }^{*}$ Univ. Grenoble Alpes, CNRS, LJK, 38000 Grenoble, France.

E-mail: corinne . berzin@univ- grenoble-alpes . $f r$ http://www-ljk.imag.fr/membres/Corinne.Berzin/
} 
some level functionals of its level curve $\mathrm{C}_{T_{n}, X}(u)$ for a fixed level $u$, say $\mathrm{C}_{T_{n}, X}(u):=$ $\left\{t \in T_{n}: X(t)=u\right\}$. The set $T_{n}$ is a bounded square of $\mathbb{R}^{2}$, having the following shape ]$-n, n\left[^{2}\right.$, with $n$ a positive integer. We are interested in the asymptotic as the square $T_{n}$ tends to $\mathbb{R}^{2}$. The eigenvector directions $v_{1}, v_{2}$ forming an orthonormal basis of $\mathbb{R}^{2}$ and the associated eigenvalues $\lambda_{1}, \lambda_{2}\left(0<\lambda_{2} \leqslant \lambda_{1}\right)$ specify the affinity $A$. Also $0<\lambda \leqslant 1$ is defined as the quotient of the eigenvalues, $\lambda:=\lambda_{2} / \lambda_{1}$. Saying that the process $X$ is isotropic means that $\lambda=1$ (see Adler and Taylor [2, Section 5.7]). A fixed unitary vector of $\mathbb{R}^{2}$ being fixed, say $v^{\star}$, it can always be written in the previous basis as:

$$
v^{\star}=\cos (\theta) v_{1}+\sin (\theta) v_{2}
$$

It is always possible to choose $-\frac{\pi}{2}<\theta \leqslant \frac{\pi}{2}$ (see Figure 1, page 40). We use Wschebor estimators [32] of the affinity parameters, defined as $\lambda$ and $\theta$, based on the shape of the level curve $\mathrm{C}_{T_{n}, X}(u)$ corresponding to a given level $u$. Also, a lot of papers have been devoted to the study of two-dimensional deformed fields, these later naturally model spatial and physical phenomena neither isotropic nor stationary. They are obtained by deforming a fixed isotropic field, say $Z: \mathbb{R}^{2} \rightarrow \mathbb{R}$, thanks to a deterministic function, say $\Upsilon: \mathbb{R}^{2} \rightarrow \mathbb{R}^{2}$, that transforms bijectively the index set. This model of deformed fields, say $X_{\Upsilon}:=Z \circ \Upsilon$, was introduced by Sampson and Guttorp in [29] but with only a stationary assumption on $Z$. Among the papers that focus on the study of these fields, let us quote Allard et al. [3], Anderes et al. [4], Anderes and Stein [5], Fouedjio et al. [16], Fournier [17] and Perrin and Senoussi [27]. For example, in [27] the authors studied the models through the covariance function. In this vein a large number of authors was interested in estimate deformation $\Upsilon$ thanks to the observation of the deformed field $X_{\Upsilon}$. As precursors, Sampson and Guttorp in [29] used several observations on a sparse grid. Another approach was then to use only one observation of $X_{\Upsilon}$ on a dense grid, we can cite for example the relevant research of [4], [5] and [16]. Notice that in [3], the authors focus on the case where the deformation $\Upsilon$ is linear which will also be our aim. The matrix representation of this deformation being the product of a diagonal and a rotation matrix, the authors call the produced effect, "geometric anisotropy". However, as far as we know, very little research is based on the use of level sets of the observed process $X_{\Upsilon}$ and except for [32] those quoted previously are not an exception to the rule. In some cases, we may be interested in the use of the information given by some functional of level sets of the process rather than given by a simple realization of the process itself. This is done for example in [17] where the author chose as particular functional the Euler characteristic of some of excursion sets of the deformed field. The covariance function of the underlying field $Z$ and the deformation $\Upsilon$ are unknown. The problem consists in identifying $\Upsilon$ using sparse data, that is through observations of some excursion sets of $X_{\Upsilon}$ above fixed levels by focusing on the mean Euler characteristic of the excursion sets for multiple windows of observation. For this doing some assumptions on $Z$ are required, among others a Gaussian property is asked. The ambition of Fournier was to identify the deformation $\Upsilon$. However, it had proved impossible to distinguish between $\Upsilon$ and another deformation $\widetilde{\Upsilon}$ if the random fields $X_{\Upsilon}$ and $X_{\widetilde{\Upsilon}}$ have the same law. Indeed and because of the property of isotropy for process $Z$, this case occurs when $\widetilde{\Upsilon}=\rho \circ \Upsilon+x$, where $\rho$ is a rotation of $\mathbb{R}^{2}$ and $x$ a vector of $\mathbb{R}^{2}$. Thus, the author almost entirely identifies $\Upsilon$ by proposing an identification of the matrix parameter $\Upsilon$ up to composition with an unknown rotation and with a translation. Also, in a Note [9], Berzin and Iribarren proposed estimators for the spectral matrix $\Sigma$ of the second order moments for a stationary and centred Gaussian bi-parametric random field. The estimators are defined as functionals over the level curves of the field and are inspired from those given by Cabaña [12] to estimate the affinity parameters previously defined. Making so, the used techniques could allow to estimate the ratio $\sqrt{\frac{\lambda_{-}}{\lambda_{+}}}$, where $0<\lambda_{-} \leqslant \lambda_{+}$are the 
eigenvalues of $\Sigma$. This last ratio being nothing else that $\lambda=\frac{\lambda_{2}}{\lambda_{1}}$ when $X=Z \circ A$ is an affine process. We see here a posteriori that inference based on one level set by the pioneering work of Cabaña could already have been useful at this time to estimate the affinity parameter $\lambda$.

Concerning the tests for the hypotheses of isotropy of general random fields, many methods have been provided. An overview of methods available to test the isotropy have been very well summarized in Weller et al. [31] but as previously explained, to my knowledge except the one given by Cabaña [12], none used the observation of the level curves. The author's basic idea was to induce a distortion in the domain that breaks isotropy. This deformation becoming apparent in the length of the level sets. This last quoted work has deeply inspired the present paper. In [12], according to his own words, the author defines the class of affine processes as a reasonable alternative to test the null hypothesis that a given almost surely differentiable stationary real process with parameter in $\mathbb{R}^{2}$ is isotropic. More precisely Cabaña, considers an affine process $\left\{X(t), t \in \mathbb{R}^{2}\right\}$ that is equal in law to $\left\{Z(A . t), t \in \mathbb{R}^{2}\right\}$, where $Z$ is isotropic (no necessarily Gaussian) and $A: \mathbb{R}^{2} \rightarrow \mathbb{R}^{2}$ is a linear self-adjoint transformation. The angle $\theta_{0}$ defining the eigenvector directions $\left(\cos \left(\theta_{0}\right), \sin \left(\theta_{0}\right)\right)$ and $\left(-\sin \left(\theta_{0}\right), \cos \left(\theta_{0}\right)\right)$ and the respective eigenvalues $\lambda_{1}, \lambda_{2}$ specify the affinity $A$. As explained by the author there is no loss of generality in assuming $\lambda_{1} \geqslant \lambda_{2}>0$ and $\lambda_{1} \lambda_{2}=1$. The statistics used are based on the size and shape of the random surface $X$, corresponding to a fixed level $u$. The regions where $X$ is observed are the indefinitely increasing family of rectangles $R_{\varrho}:=\{\varrho t: t \in R\}, \varrho \in \mathbb{R}^{+}$obtained from a fixed rectangle $R \subset \mathbb{R}^{2}$. In order that the level sets $\mathcal{C}_{\mathrm{R}_{\varrho}, \mathrm{X}}(u):=\left\{s \in R_{\varrho}: X(s)=u\right\}$ are curves, some restrictions had to be imposed to the process $X$, (see Proposition 2.1 for the discussion about the topic). So, the paths of $X$ are required to be of class $\mathbf{C}^{2}$ on $\mathbb{R}^{2}$. Also the pair $(X(0), \nabla X(0))$ is supposed to have a joint density $p_{X(0), \nabla X(0)}\left(x, x^{\prime}\right)$ in $\mathbb{R} \times \mathbb{R}^{2}$, bounded for $x^{\prime}$ varying in a compact subset of $\mathbb{R}^{2}$ and $x$ in some neighbourhood of $u, \nabla X$ standing for the gradient of $X$. The stationary random fields $\Theta$ and $\|\nabla X\|_{2}$ are defining the polar representation of $\nabla X$, that is for $t \in \mathbb{R}^{2}, \nabla X(t)=\|\nabla X(t)\|_{2}(\cos (\Theta(t)), \sin (\Theta(t)))$, where $\|\cdot\|_{2}$ stands for the Euclidean norm in $\mathbb{R}^{2}$.

In the first part of his paper, Cabaña proposes estimators of the affinity parameters defined as $k:=\left(1-\lambda^{2}\right)^{\frac{1}{2}}$ with $\lambda:=\frac{\lambda_{2}}{\lambda_{1}}$ and $\theta_{0}$, based on the $u$ level curve of $X$ in the following way. First, he defines for $f:]-\pi, \pi] \rightarrow \mathbb{R}$, a bounded and measurable function, the general line integral

$$
\mathcal{F}(f, R):=|R|^{-1} \int_{\mathcal{C}_{\mathrm{R}, \mathrm{X}}(u)} f(\Theta(t)) \mathrm{d} \sigma_{1}(t),
$$

where $|R|$ denotes the area of $R$ that is $\sigma_{2}(R)$, and for $d=1,2, \sigma_{d}$ denotes the Lebesgue measure on $\mathbb{R}^{d}$. (Note that the assumptions made on $X$ ensure that these line integrals are well defined, see the beginning of the paragraph General level functionals in Section 2.1 page 11 for a commentary about this topic).

Then he defines three statistics obtained as particular line integrals, let

$$
\begin{aligned}
\mathcal{L}(R) & :=|R|^{-1} \int_{\mathcal{C}_{\mathrm{R}, \mathrm{X}}(u)} 1 \mathrm{~d} \sigma_{1}(t), \\
\mathcal{C}(R) & :=|R|^{-1} \int_{\mathcal{C}_{\mathrm{R}, \mathrm{X}}(u)} \cos (2 \Theta(t)) \mathrm{d} \sigma_{1}(t), \\
\mathcal{S}(R) & :=|R|^{-1} \int_{\mathcal{C}_{\mathrm{R}, \mathrm{x}}(u)} \sin (2 \Theta(t)) \mathrm{d} \sigma_{1}(t) .
\end{aligned}
$$

The author states that assumptions made on process $X$ imply that these functionals have first and second moments, that is, Rice formulas of orders one and two are ensured. Thus, 
he proves that the affinity parameters previously defined as $k$ and $\theta_{0}$ can be obtained via the straightforward computations of $\mathbb{E}[\mathcal{L}(R)], \mathbb{E}[\mathcal{C}(R)]$ and of $\mathbb{E}[\mathcal{S}(R)]$. In this way, the author highlights the fact that

$$
\tan \left(2 \theta_{0}\right)=\mathbb{E}[\mathcal{S}(R)] / \mathbb{E}[\mathcal{C}(R)] \text { and } g(k):=\sqrt{(\mathbb{E}[\mathcal{C}(R)])^{2}+(\mathbb{E}[\mathcal{S}(R)])^{2}} / \mathbb{E}[\mathcal{L}(R)],
$$

where $g$ is a known continuously increasing function on $[0,1]$, such that $g(0)=0$.

In view of studying the asymptotic properties of future estimators of $k$ and $\theta_{0}$ that will naturally result of the two above equalities, the author adds a condition of uniform mixing for process $X$ that we recall here. The field $X$ is said to be uniformly mixing, when there exists a decreasing function $\delta: \mathbb{R}^{+} \rightarrow \mathbb{R}^{+}, \lim _{y \rightarrow+\infty} \delta(y)=0$, such that if $S, S^{\prime}$ are two Borel sets in $\mathbb{R}^{2}, d:=\operatorname{distance}\left(S, S^{\prime}\right), \sigma(S)$ is the $\sigma$-fields generated by $\{X(t): t \in S\}$, then for any events $A \in \sigma(S), B \in \sigma\left(S^{\prime}\right)$, the inequality $|\mathbb{P}(A / B)-\mathbb{P}(A)| \leqslant \delta(d)$ holds. Under the uniform mixing condition for $X$, Iribarren [19] has shown that the asymptotic distribution of $\varrho|R|^{\frac{1}{2}}(\mathcal{F}(f, \varrho R)-\mathbb{E}[\mathcal{F}(f, R)])$ (as $\varrho$ goes to infinity) is Gaussian with mean zero and variance $\sigma_{f}^{2}$.

This leads Cabaña to conclude that the estimators $\widehat{\theta}_{0, \varrho}$ of $\theta_{0}$ and $\widehat{k}_{\varrho}$ of $k$ defined as $\widehat{\theta}_{0, \varrho}:=(1 / 2) \arg (\mathcal{S}(\varrho R) / \mathcal{C}(\varrho R))$ and $\widehat{k}_{\varrho}:=g^{-1}\left(\sqrt{\mathcal{C}^{2}(\varrho R)+\mathcal{S}^{2}(\varrho R)} / \mathcal{L}(\varrho R)\right)$ are consistent in probability.

In the second part of his work, by pointing out that an isotropic field $X$ has $k=0$ (see [2, Section 5.7]) and the farther is $X$ from isotropy, the greater is $k$, then he motivates two isotropy tests for the null hypotheses " $X$ is isotropic" versus the alternative " $X$ is affine", as follows. For the first test that will be truthfully our main focus, he first establishes that under the null hypothesis, the random variables $\mathcal{C}(\varrho R), \mathcal{S}(\varrho R)$ and $\mathcal{L}(\varrho R)$ are asymptotically independent and also that $\mathbb{E}[\mathcal{C}(R)]=\mathbb{E}[\mathcal{S}(R)]=0$. He deduces from this result that $\varrho^{2}|R|\left(\mathcal{C}^{2}(\varrho R)+\mathcal{S}^{2}(\varrho R)\right)$ is asymptotically distributed as $\sigma_{0}^{2} \chi_{2}^{2}$, where $\sigma_{0}^{2}$ denotes the common value of $\sigma_{f}^{2}$ when $f(\theta)$ is $\cos (2 \theta)$ or $\sin (2 \theta)$. This last result suggests using the test variable

$$
\mathcal{A}_{\varrho}(R):=\varrho^{2}|R| \frac{\mathcal{C}^{2}(\varrho R)+\mathcal{S}^{2}(\varrho R)}{\mathcal{L}^{2}(\varrho R)}
$$

to define the rejection region as $\mathcal{A}_{\varrho}(R)>\gamma$ for the null hypothesis " $k=0$ ". Under $k=0$, the asymptotic distribution of $\mathcal{A}_{\varrho}(R)$ is $\sigma_{0}^{2}(\mathbb{E}[\mathcal{L}(R)])^{-2} \chi_{2}^{2}$, since $\mathcal{L}(\varrho R)$ converges in probability to $\mathbb{E}[\mathcal{L}(R)]$. Furthermore when $k \neq 0, \varrho^{-2}|R|^{-1} \mathcal{A}_{\varrho}(R)$ converges in probability to $g^{2}(k)>0$, and then $\mathcal{A}_{\varrho}(R)$ is stochastically unbounded for $\varrho$ tending to infinity. Thus, the proposed critical region provides a consistent test for any positive constant $\gamma$.

Nevertheless, as previously announced, the author develops a second isotropy test, an Ftest, to remedy the imperfections of the first proposed test. This second test is still based on the level sets of $X$ and ensues from the following argumentation. In the case where the null hypothesis completely specifies the distribution of the process, then $\sigma_{0}^{2}$ and $\mathbb{E}[\mathcal{L}(R)]$ can be evaluated since they only depend on the distribution of the subjacent process $Z$ and on $\lambda_{1}$ that worth 1 since $k=0$ and $\lambda_{1} \lambda_{2}=1$. Thus, in this case, all the ingredients are gathered to perform the asymptotic test. But this is rarely actually the case, since before deciding if the isotropic model is suitable, an estimation of joint distribution of the process is necessary beforehand. Thus Cabaña developed a one-way analysis of variance test in the following manner. The rectangle $R$ is still fixed and is cut into $n$ rectangles of equal area, forming a partition $\mathcal{F}:=\left\{R_{1}, R_{2}, \ldots, R_{n}\right\}$. Under the same uniform mixing condition as supposed in the first part of his paper, the previous cited work [19] also allows to establish that since $R_{1}, R_{2}, \ldots, R_{n}$ are disjoint then $\varrho\left|R_{i}\right|^{\frac{1}{2}}\left(\mathcal{C}\left(\varrho R_{i}\right)-\mathbb{E}\left[\mathcal{C}\left(R_{i}\right)\right]\right)$, $\varrho\left|R_{i}\right|^{\frac{1}{2}}\left(\mathcal{S}\left(\varrho R_{i}\right)-\mathbb{E}\left[\mathcal{S}\left(R_{i}\right)\right]\right), i=1,2, \ldots, n$ are asymptotically independent. Thus, the analysis of the model

$$
\mathcal{C}\left(\varrho R_{i}\right)=\mathbb{E}\left[\mathcal{C}\left(R_{i}\right)\right]+\text { error, } \mathcal{S}\left(\varrho R_{i}\right)=\mathbb{E}\left[\mathcal{S}\left(R_{i}\right)\right]+\text { error }(i=1,2, \ldots, n)
$$


suggested the test variable

$$
\begin{aligned}
\mathcal{B}_{\varrho}(\mathcal{F}) & :=\frac{n^{-1}\left(\sum_{i=1}^{n} \mathcal{C}\left(\varrho R_{i}\right)\right)^{2}+n^{-1}\left(\sum_{i=1}^{n} \mathcal{S}\left(\varrho R_{i}\right)\right)^{2}}{\sum_{i=1}^{n} \mathcal{C}^{2}\left(\varrho R_{i}\right)+\sum_{i=1}^{n} \mathcal{S}^{2}\left(\varrho R_{i}\right)-n^{-1}\left(\sum_{i=1}^{n} \mathcal{C}\left(\varrho R_{i}\right)\right)^{2}-n^{-1}\left(\sum_{i=1}^{n} \mathcal{S}\left(\varrho R_{i}\right)\right)^{2}} \\
& =\frac{n\left(\mathcal{C}^{2}(\varrho R)+\mathcal{S}^{2}(\varrho R)\right)}{\sum_{i=1}^{n}\left(\mathcal{C}^{2}\left(\varrho R_{i}\right)+\mathcal{S}^{2}\left(\varrho R_{i}\right)\right)-n\left(\mathcal{C}^{2}(\varrho R)+\mathcal{S}^{2}(\varrho R)\right)} .
\end{aligned}
$$

Under the hypothesis " $k=0$ ", the law of $\mathcal{B}_{\varrho}(\mathcal{F})$ tends to that of a $F_{2,2 n-2}$ distribution as $\varrho$ goes to infinity. When $k \neq 0$, that is when $X$ is an affine process but not isotropic, the limit law is a noncentral $F$-distribution. The critical region is then $\mathcal{B}_{\varrho}(\mathcal{F})>F_{2,2 n-2}(\alpha)$, where $F_{2,2 n-2}(\alpha)$ is the $(1-\alpha)$-quantile of the $F_{2,2 n-2}$ distribution.

As ultimate remark Cabaña specifies that the size of the rectangles $R_{i}$ has to be large enough to ensure a fair approximation to the limit distribution. Doing so necessarily limits the value of the number $n$ of rectangles of the partition.

Bearing in mind to estimate the affinity parameters $\lambda$ and $\theta$ defined at the beginning of this Introduction, by using the level sets of the process $X_{\Upsilon}$ and in the case where the deformation $\Upsilon:=A$ is a linear self-adjoint and definite positive one, our starting point was an example from [32, Chap 3.6, Paragraph F, page 79].

In this example Wschebor, proposes estimators of the affinity parameters of a process $X$ indexed in $\mathbb{R}^{2}$ based on the shape of its level curve, corresponding to a given level $u$. The author explains as preamble that his work was largely based on Cabaña [12], although his estimators were different. More precisely, Wschebor considers $\{X(t), t \in$ $\left.\mathbb{R}^{2}\right\}$ a $\mathbf{C}^{2}$-affine process (not necessarily Gaussian) and fixes $u$ a level in $\mathbb{R}$ supposed to be a regular value of $X$, (see Proposition 2.1 for much details on sufficient hypothesis ensuring such a property). Moreover, the process $X$ is supposed to be sufficiently regular in order to verify the one and second order Rice formula. Chapter 3 of the book gives many details about the topic and on particular hypotheses $H_{1,2}$ and $H_{2,2}$. Furthermore the process $X$ is supposed satisfying a condition of $\eta$-dependence. We recall here this notion. The process $(X, \nabla X)$ is said to be $\eta$-dependent if $\left\|t_{i}-t_{j}^{\prime}\right\|_{2} \geqslant \eta>0(i=1, \ldots, k, j=$ $1, \ldots, \ell, k, \ell=1,2, \ldots)$, then the random vectors $\left(\left(X\left(t_{1}\right), \nabla X\left(t_{1}\right)\right), \ldots,\left(X\left(t_{k}\right), \nabla X\left(t_{k}\right)\right)\right)$, $\left(\left(X\left(t_{1}^{\prime}\right), \nabla X\left(t_{1}^{\prime}\right)\right), \ldots,\left(X\left(t_{\ell}^{\prime}\right), \nabla X\left(t_{\ell}^{\prime}\right)\right)\right)$ are independent. Notice that the condition of $\eta$ dependence for $X$ implies that of uniformly mixing required by Cabaña in [12]. The regions where $X$ is observed are the indefinitely increasing family of rectangles $R_{\varrho}:=$ $\left\{\left(\varrho_{1} t_{1}, \varrho_{2} t_{2}\right):\left(t_{1}, t_{2}\right) \in R\right\}$, if $\varrho:=\left(\varrho_{1}, \varrho_{2}\right), \varrho_{1}, \varrho_{2} \in \mathbb{R}^{+}$obtained from a fixed rectangle $R \subset \mathbb{R}^{2}$. By defining the following general functional $\mathcal{J}(f, R)$ of the fixed level $u$ by:

$$
\mathcal{J}(f, R):=|R|^{-1} \int_{\mathcal{C}_{\mathrm{R}, \mathrm{X}}(u)} f\left(\frac{\nabla X(t)}{\|\nabla X(t)\|_{2}}\right) \mathrm{d} \sigma_{1}(t),
$$

for $f: S^{1} \rightarrow \mathbb{R}$ or $f: S^{1} \rightarrow \mathbb{R}^{2}$, a bounded function where $S^{1}$ is the boundary of the unit ball of $\mathbb{R}^{2}$, the author considers two particulars functionals of the level set $u$. The first one where $f \equiv \mathbf{1}$ corresponds to the particular functional being the measure of dimensional area of the level set by unit of volume, say $\mathcal{J}(\mathbf{1}, R)$. The second one, say $\mathcal{J}\left(\overrightarrow{f^{\star}}, R\right)$, corresponds to the function $\overrightarrow{f^{\star}}$ taking values in $\mathbb{R}^{2}$, that will be evaluated in the value of the normalized gradient of $X$ and being its value if this one lives in the same half-plane as that of $v^{\star}$, a fixed unitary vector in $\mathbb{R}^{2}$, and minus this value if not, that is $\overrightarrow{f^{\star}}(\omega):=\omega \times\left(\mathbb{1}_{\left\{\left\langle\omega, v^{\star}\right\rangle \geqslant 0\right\}}-\mathbb{1}_{\left\{\left\langle\omega, v^{\star}\right\rangle<0\right\}}\right)$ if $\omega$ lives in $S^{1}$. Remark that function $\overrightarrow{f^{\star}}$ is continuous except on points $\left(-v_{2}^{\star}, v_{1}^{\star}\right)$ and $\left(v_{2}^{\star},-v_{1}^{\star}\right)$, where $v_{i}^{\star}, i=1,2$, are the coordinates of the vector $v^{\star}$. Note that with the notations of Cabaña, this functional can be expressed as

$$
\mathcal{J}\left(\overrightarrow{f^{\star}}, R\right)=|R|^{-1} \int_{\mathcal{C}_{\mathrm{R}, \mathrm{X}}(u)}(\cos (\Theta(t)), \sin (\Theta(t))) \operatorname{sign}\left(\cos \left(\Theta(t)-\theta_{1}\right)\right) \mathrm{d} \sigma_{1}(t),
$$


if $v^{\star}=\left(\cos \left(\theta_{1}\right), \sin \left(\theta_{1}\right)\right)$.

Under the hypotheses that $\inf \left(\varrho_{1}, \varrho_{2}\right) \rightarrow+\infty$, Wschebor showed that the ratio $\mathcal{Q}\left(R_{\varrho}\right):=$ $\frac{\mathcal{J}\left(\overrightarrow{f^{*}}, R_{\varrho}\right)}{\mathcal{J}\left(\mathbf{1}, R_{\varrho}\right)}$ tends in probability towards $\frac{\mathbb{E}\left[\mathcal{J}\left(\overrightarrow{f^{*}}, R\right)\right]}{\mathbb{E}[\mathcal{J}(\mathbf{1}, R)]}$. This convergence enabled him to propose probability consistent estimators of direction $\theta$, say $\widehat{\theta}_{\varrho}$ and also of $\lambda$, say $\widehat{\lambda}_{\varrho}$, based on the observation of the quotient $\mathcal{Q}\left(R_{\varrho}\right)$. More precisely by using the one order Rice formula, he proves that the ratio $\frac{\mathbb{E}\left[\mathcal{J}\left(\vec{f}^{\star}, R\right)\right]}{\mathbb{E}[\mathcal{J}(1, R)]}$ can be written in the fixed direct orthonormal basis $\left(v^{\star}, v^{\star \star}\right)$ (see Figure 1 page 40 ) as $F_{1}(\lambda, \theta) v^{\star}+F_{2}(\lambda, \theta) v^{\star \star}$, while functions $F_{i}$, $i=1,2$ are explicitly known (see equation (5.5) page 41). Thus, his idea was to observe the ratio $\mathcal{Q}\left(R_{\varrho}\right)$ in the fixed basis $\left(v^{\star}, v^{\star \star}\right)$, say $\left(X_{\varrho}, Y_{\varrho}\right)$. Following this way Wschebor has shown that the system of equations

$$
\begin{cases}X_{\varrho} & =F_{1}(\lambda, \theta) \\ Y_{\varrho} & =F_{2}(\lambda, \theta)\end{cases}
$$

admits one and only one solution, say $\left.\left.\left(\widehat{\lambda}_{\varrho}, \widehat{\theta}_{\varrho}\right) \in\right] 0,1[\times]-\frac{\pi}{2}, \frac{\pi}{2}\right]$ as long as $\left(X_{\varrho}, Y_{\varrho}\right)$ is such that $X_{\varrho} \neq \frac{2}{\pi}$ or $Y_{\varrho} \neq 0$ (see Figure 2 page 42 and Figure 3 page 43). It is important to note that such an approach furnished estimators $\left(\widehat{\lambda}_{\varrho}, \widehat{\theta}_{\varrho}\right)$ of the affinity parameters $(\lambda, \theta)$ in an implicitly manner which is not the case for those provided by Cabaña, the latter not being linked together and can therefore be calculated independently of each other. Then the author claims that the derived estimators are consistent in probability. Note that this consistence property is valid under the hypothesis that $0<\lambda<1$ and that $-\frac{\pi}{2}<\theta<\frac{\pi}{2}$, that is for $(\lambda, \theta)$ belonging to an open set. Nothing is suggested by the author in case where the parameters $(\lambda, \theta)$ belong to the boundary of the set; neither for the convergence rate of these estimators, nor concerning those of Cabaña's paper. Moreover, Wschebor does not suggest any isotropic test since in fact his example was part of those intended to illustrate the Rice formula and was not there intended to be dealt with in details.

Thus, our goal was to revisit Wschebor example in [32] by showing among other things the almost sure convergence of the estimators $\left(\widehat{\lambda}_{\varrho}, \widehat{\theta}_{\varrho}\right)$ towards the true parameters $(\lambda, \theta)$, by exhibiting their rate of convergence via a central limit theorem, and also by paying particular attention to the case where the parameters live on the border of their definition domain. That naturally leads to propose an isotropic test by considering the ideas developed in Cabaña [12].

Main contribution of the paper In the present work following the way opened by Cabaña in [12], we still work with a $\mathbf{C}^{2}$-affine process, say $\left\{X(t), t \in \mathbb{R}^{2}\right\}$, that is equal in law to $\left\{Z(A . t), t \in \mathbb{R}^{2}\right\}$, where $Z$ is isotropic and $A: \mathbb{R}^{2} \rightarrow \mathbb{R}^{2}$ is a linear self-adjoint transformation, additionally supposed to be a positive definite one. We get rid of the $\eta$-dependence hypothesis for the process $X$ requested by Wschebor in [32] and we add the hypothesis that the subjacent process $Z$ is Gaussian and some technical assumptions on its covariance. We consider his proposed estimators of the affinity parameters $\lambda$ and $\theta$ by considering the particular following situation. We select the fixed rectangle $R:=]-1,1[\times]-1,1\left[\right.$, the sequence $\varrho=\left(\varrho_{1}, \varrho_{2}\right):=(n, n)$ with $n$ positive integer, and we consider the observation windows $\left.T_{n}:=R_{\varrho}=\right]-n, n\left[^{2}\right.$ with $n$ tending towards infinity. For these observation windows and as previously explained in the second part of the Introduction we define the following general functional of the fixed level $u \in \mathbb{R}$

$$
J_{f}^{(n)}(u):=\frac{1}{(2 n)^{2}} \int_{\mathcal{C}_{\mathrm{T}_{n}, \mathrm{X}}(u)} f\left(\frac{\nabla X(t)}{\|\nabla X(t)\|_{2}}\right) \mathrm{d} \sigma_{1}(t),
$$

for $f$ in $\mathcal{B}_{k}, k \in \mathbb{N}^{\star}$, the class of functions $f$ such that: $f: S^{1} \rightarrow \mathbb{R}^{k}$, is measurable, bounded, continuous except maybe on a finite number of points of the sphere $S^{1}$. We 
observe the ratio $\mathcal{Q}\left(T_{n}\right):=\frac{J_{f^{\star}}^{(n)}(u)}{J_{1}^{(n)}(u)}$ in the fixed basis $\left(v^{\star}, v^{\star \star}\right)$, say $\left(X_{n}, Y_{n}\right)$. Considering the system of equations (1.1), in the same way as proposed by Wschebor we construct in an implicit manner the estimators $\left(\widehat{\lambda}_{n}, \widehat{\theta}_{n}\right)$ (see Proposition 5.10).

Our main contribution consists, in the one hand, to establish the almost sure consistence of these estimators (Theorem 5.11) in case where $0<\lambda<1$ and $-\frac{\pi}{2}<\theta<\frac{\pi}{2}$. We need to prove Theorem 4.1 stated below using an ergodic theorem (Adler $[1, \S 6.5]$ ) and Rice formula (see the seminal work of Rice [28]).

Theorem 4.1 For $f: S^{1} \rightarrow \mathbb{R}$ a function belonging to $\mathcal{B}_{1}$,

$$
J_{f}^{(n)}(u) \underset{n \rightarrow+\infty}{\stackrel{a . s .}{\longrightarrow}} \mathbb{E}\left[J_{f}^{(1)}(u)\right] .
$$

This theorem applied to the particular functions $\overrightarrow{f^{\star}}$ and to the function 1 taking values in $\mathbb{R}$ and identically equal to one implies that,

$$
\frac{J_{\overrightarrow{f^{\star}}}^{(n)}(u)}{J_{\mathbf{1}}^{(n)}(u)}=X_{n} v^{\star}+Y_{n} v^{\star \star} \underset{n \rightarrow+\infty}{\stackrel{a . s .}{\longrightarrow}} \frac{\mathbb{E}\left[J_{\overrightarrow{f^{\star}}}^{(1)}(u)\right]}{\mathbb{E}\left[J_{\mathbf{1}}^{(1)}(u)\right]}=F_{1}(\lambda, \theta) v^{\star}+F_{2}(\lambda, \theta) v^{\star \star},
$$

where the functions $F_{1}, F_{2}$ are defined by equation (5.5) page 41 (Proposition 5.2). By noting $\vec{F}:=\left(F_{1}, F_{2}\right)$ and by using the fact that $\vec{F}$ is a $\mathbf{C}^{2}$-diffeomorphism (Proposition $5.5)$, we deduce from this theorem the almost sure convergence of $\left(X_{n}, Y_{n}\right)=\vec{F}\left(\widehat{\lambda}_{n}, \widehat{\theta}_{n}\right)$ towards $\vec{F}(\lambda, \theta)$ and then of that of $\left(\widehat{\lambda}_{n}, \widehat{\theta}_{n}\right)$ towards $(\lambda, \theta)$ provided that these last parameters live in an open set.

In the second hand our contribution was to propose a Central Limit Theorem (CLT) (Theorem 5.17) for those estimators and some confidence intervals (Corollary 5.18), still in case where $0<\lambda<1$ and $-\frac{\pi}{2}<\theta<\frac{\pi}{2}$. For proving the asymptotic normality of the estimators, we use a CLT for general functionals $J_{f}^{(n)}(u)$, that was the subject of Theorem 4.7 stated here.

Theorem 4.7 For $f: S^{1} \rightarrow \mathbb{R}$ a function belonging to $\mathcal{B}_{1}$, we have the following convergence,

$$
\xi_{f}^{(n)}(u):=2 n\left(J_{f}^{(n)}(u)-\mathbb{E}\left[J_{f}^{(n)}(u)\right]\right) \underset{n \rightarrow+\infty}{\stackrel{L a w}{\longrightarrow}} \mathcal{N}\left(0 ; \Sigma_{f, f}(u)\right),
$$

where $\Sigma_{f, f}(u)$ is defined by (4.2).

We deduce from Theorems 4.1 and 4.7 the following proposition.

\section{Proposition 5.12}

$$
2 n\left(\frac{J_{\overrightarrow{f^{\star}}}^{(n)}(u)}{J_{\mathbf{1}}^{(n)}(u)}-\frac{\mathbb{E}\left[J_{\overrightarrow{f^{\star}}}^{(1)}(u)\right]}{\mathbb{E}\left[J_{\mathbf{1}}^{(1)}(u)\right]}\right) \underset{n \rightarrow+\infty}{\stackrel{L a w}{\longrightarrow}} \mathcal{N}\left(0 ; \Sigma^{\star}(u)\right) .
$$

And writing this last convergence result in the basis $\left(v^{\star}, v^{\star \star}\right)$, gives that

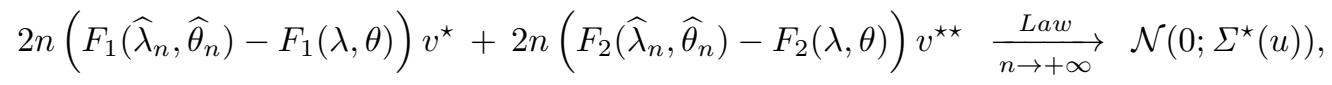

so that

$$
2 n\left(\vec{F}\left(\widehat{\lambda}_{n}, \widehat{\theta}_{n}\right)-\vec{F}(\lambda, \theta)\right) \underset{n \rightarrow+\infty}{\stackrel{L a w}{\longrightarrow}} \mathcal{N}\left(0 ; \Sigma_{Q}^{\star}(u)\right),
$$


where $\Sigma_{Q}^{\star}(u):=Q \times \Sigma^{\star}(u) \times Q^{t}$ and $Q$ is the change of basis matrix from the canonical basis $(\vec{i}, \vec{j})$ to the basis $\left(v^{\star}, v^{\star \star}\right)$.

Once again by using the fact that $\vec{F}$ is a $C^{2}$-diffeomorphism, we deduce a CLT for estimators $\left(\widehat{\lambda}_{n}, \widehat{\theta}_{n}\right)$, as soon as the affinity parameters $\lambda$ and $\theta$ do not belong to the edges of the parameter space.

Remark: this method for estimating the parameters $(\lambda, \theta)$ is available only when the space parameters for $X$ is $\mathbb{R}^{2}$. Indeed, when this space is $\mathbb{R}^{d}(d \geqslant 2)$, we deal with a function $\vec{F}$ taking values from $\mathbb{R}^{2 d-2}$ into $\mathbb{R}^{d}$. The arguments of $\vec{F}$ would be $\left(\mu_{2}, \ldots, \mu_{d}, \theta_{1}, \ldots, \theta_{d-1}\right), \mu_{i}$ being the ratio of the decreasing eigenvalues $\lambda_{i}$ of $A$, let $\mu_{i}:=\lambda_{i} / \lambda_{1}, i=2, d$, and $\theta_{j}$ the angles involved in the parametrization in spherical coordinates of vector $v^{\star}, j=1, d-1$. The arrival space of function $\vec{F}$ is $\mathbb{R}^{d}$ since the gradient $\nabla X$ lives into $\mathbb{R}^{d}$. Thus, if we want the function $\vec{F}$ to be an isomorphism we must require the following condition on $d$, that is $2 d-2=d$, which is only satisfied when $d=2$.

The tools for proving the CLT in Theorem 4.7 is the use of the CLT technique for functionals belonging to the Wiener-Itô chaos. This method has been developed by Nourdin et al. [24], Nualart et al. [25] and Peccati and Tudor [26] among others. The idea for proving such a CLT is inspired by the precursor work of Kratz and León [20]. In the case where the process $X$ is a stationary Gaussian isotropic process indexed by $\mathbb{R}^{2}$ and the observation window is a fixed bounded rectangle $T$, the authors propose a way to approximate $\sigma_{1}\left(\mathcal{C}_{\mathrm{T}, \mathrm{X}}(u)\right) / \sigma_{2}(T)$, say $J_{\mathbf{1}}(u):=\mathcal{J}(\mathbf{1}, T)$, by other functionals $J_{\mathbf{1}}(u, \sigma)$ $\left(\sigma \rightarrow 0\right.$ ) with the help of a kernel $K_{\sigma}$ tending to the delta-Dirac function in $u$. This is done in such a way that the approximating functional can be expressed as stochastic integrals with respect to Hermite polynomials. It consists in using the coarea formula (see Federer [15, Theorem 3.2.12 p 249] and also Berzin et al. [10, Corollary 2.1.1]), for $J_{1}(u, \sigma)$, transforming then this functional initially expressed on the level curve as a temporal functional on the rectangle $T$ and getting then its Hermite expansion in $H(X)$ the space of real square integral functionals of the field $X$. Using this technique in the case where the observation window is still a fixed bounded rectangle $T$, as $\sigma \rightarrow 0$ we were able to express in turn a general functional on the level curve $\mathcal{C}_{\mathrm{T}, \mathrm{X}}(u)$ as stochastic integral with respect to Hermite polynomials, let $J_{f}(u):=\mathcal{J}(f, T), f$ being a general function that belongs to the class of functions $\mathcal{B}_{1}$ (Theorem 3.5). Applying this result to the squares $T_{n}$, as $n \rightarrow \infty$, we obtain the asymptotic variance $\Sigma_{f, f}(u)$ of the centred and suitably rescaled general functionals $J_{f}^{(n)}(u)$, say $\xi_{f}^{(n)}(u)$, with (Proposition 4.3). Finally, applying the Peccati-Tudor method [26] and expressing the functionals $\xi_{f}^{(n)}(u)$ into the Wiener-Itô chaos, we obtain the CLT in Theorem 4.7. The way of proceeding is completely based on the methods developed into the paper Estrade and León [14] itself inspired by the article [20]. In this work the authors show a CLT for the Euler characteristic of the excursions above $u$ of the field $X$ on $T$ as $T$ grows to $\mathbb{R}^{d}, X$ being a stationary Gaussian isotropic process indexed in $\mathbb{R}^{d}$.

Our real contribution for proving the CLT, apart from showing the non-degeneration of the asymptotic limit matrix variance $\Sigma^{\star}(u)$ (Remark 5.14), was to rely on the two functionals $J_{f}(u)$ and $J_{f}(u, \sigma)$ its approximation via the kernel $K_{\sigma}$, that is to show that $J_{f}(u, \sigma)$ is an $L^{2}$-convergent approximation of $J_{f}(u)$ (Proposition 3.7). It was the opportunity to obtain as a first bonus the $L^{2}$-continuity in the level $u$ of the random variable $J_{f}(u)$ (Theorem 3.1), which is a very nice interesting result in itself. We did not find it in the literature and we believe that this result deserves consideration. The proof is far from obvious and implements a number of ideas developed in Berzin et al. [10], from which a local parametrization of the level set $\mathrm{C}_{T, X}(u+\delta)$ near the level $u$ (see [10, Theorem 3.1.2]). The second bonus was brought by obtaining the one order Rice formula 
for $J_{f}(u)$, that is the exact computation of $\mathbb{E}\left[J_{f}(u)\right]$ (Proposition 3.10).

Having a closer look at how the estimators were obtained, we finally show apart in the final section the almost sure convergence of estimators $\widehat{\lambda}_{n}$ (Theorem 6.1) and also their rate of convergence (Theorem 6.2) in case where the true parameters $(\lambda, \theta)$ belong to the boundary of the set $] 0,1[\times]-\frac{\pi}{2}, \frac{\pi}{2}[$.

By supposing that the covariance function of the subjacent process $Z$ is known, this leads us also to propose as in Section 2.1 of Cabaña's paper, statistical tests for the null hypothesis " $X$ is isotropic" versus the alternative " $X$ is affine" (Theorem 6.10). Those ones are suggested by the convergence result previously stated in Proposition 5.12. As explained in the first part of the Introduction testing the isotropy means to test the null hypothesis $H_{0}: \lambda=1$ against $H_{1}: \lambda<1$. Because

$$
\left(\frac{\mathbb{E}\left[J_{\overrightarrow{f^{\star}}}^{(1)}(u)\right]}{\mathbb{E}\left[J_{1}^{(1)}(u)\right]}=F_{1}(\lambda, \theta) v^{\star}+F_{2}(\lambda, \theta) v^{\star \star} \neq \frac{2}{\pi} v^{\star}\right) \Longleftrightarrow(\lambda<1),
$$

Proposition 5.12 ensures that under the hypothesis $H_{0}$,

$$
T_{\overrightarrow{f^{\star}}}^{(n)}(u):=2 n\left(\frac{J_{\overrightarrow{f^{\star}}}^{(n)}(u)}{J_{\mathbf{1}}^{(n)}(u)}-\frac{2}{\pi} v^{\star}\right) \underset{n \rightarrow+\infty}{\stackrel{L a w}{\longrightarrow}} \mathcal{N}(0 ; \Gamma(u, \tau)),
$$

(see Corollary 6.7), where the asymptotic matrix variance $\Gamma(u, \tau)$ depends only on $u$ and $\tau$ the common value of the eigenvalues of matrix $A$. Since $\mathbb{E}\left[J_{1}^{(1)}(u)\right]=\mathbf{C} \tau$ under hypothesis $H_{0}$, with $\mathbf{C}$ a computable constant, we estimate the parameter $\tau$ by an almost sure convergence estimator $\widehat{\tau}_{n}$ obtained by the application of Theorem 4.1 in the case where function $f$ is identically equal to 1 . Thus, under the hypothesis of isotropy

$$
\Xi_{\overrightarrow{f^{\star}}}^{(n)}(u):=\left(S_{\overrightarrow{f^{\star}}}^{(n)}(u)\right)^{t} S_{\overrightarrow{f^{\star}}}^{(n)}(u) \underset{n \rightarrow+\infty}{\stackrel{L a w}{\longrightarrow}} \chi_{2}^{2},
$$

where $S_{\overrightarrow{f^{\star}}}^{(n)}(u):=\Gamma^{-\frac{1}{2}}\left(u, \widehat{\tau}_{n}\right) \cdot T_{\overrightarrow{f^{\star}}}^{(n)}(u)$.

As in [12], the last convergence result naturally suggests using the test variable $\Xi_{\overrightarrow{f^{\star}}}^{(n)}(u)$. We built a consistent test with rejection region $\Xi_{f^{\star}}^{(n)}(u)>\gamma$, since the previous equivalence ensures that $\frac{1}{(2 n)^{2}} \Xi_{\overrightarrow{f^{\star}}}^{(n)}(u)$ converges in probability to $b>0$ in case of anisotropy (see Remark 6.11).

We end with a remark pinpointing the fact that such an isotropy test cannot be implemented in this way in the case where the $X$ process parameters are not in $\mathbb{R}^{2}$. In fact, Proposition 5.12 could be generalized without difficulty to the case where the parameter space is $\mathbb{R}^{d}(d \geqslant 3)$. Nevertheless, it is not enough for considering the idea of adapting the isotropy test proof to the new situation. If we refer to the previous remark made on page 8 , using the same notations, under the isotropy hypothesis the ratio $\mathbb{E}\left[J_{\overrightarrow{f^{\star}}}^{(1)}(u)\right] / \mathbb{E}\left[J_{1}^{(1)}(u)\right]$ is not characterized by equalities $\mu_{2}=\cdots=\mu_{d}=1$. Roughly speaking the isotropy hypothesis implies that $\mathbb{E}\left[J_{\overrightarrow{f^{\star}}}^{(1)}(u)\right] / \mathbb{E}\left[J_{\mathbf{1}}^{(1)}(u)\right]=c(d) v^{\star}(c(d)$ being a constant depending only of $d$ and computed under hypothesis of isotropy), but the converse is not always true. Such a test would not be then consistent.

Outline of the paper Section 2.1 contains some notations, among others definitions, affine process and isotropic process. It gives explicitly the type of general functionals on the level set $u$ we are looking for, say $J_{f}^{(n)}(u)$, where the observation window $T_{n}$ grows to $\mathbb{R}^{2}$ when $n$ goes to infinity. It is also an opportunity in this part to introduce some 
concepts, when the observation window is a fixed rectangle $T$ of $\mathbb{R}^{2}$. They deal with regularity properties of level sets and Rice formulas, that are closed formulas for the first and second moments for functionals defined on the level set, say $J_{f}(u)$. Section 2.2 gives the assumptions on the interest model and some examples of processes satisfying these assumptions.

In Section 3 the observation window is fixed by taking an open bounded rectangle $T$ of $\mathbb{R}^{2}$. We begin by showing in Section 3.1 the $L^{2}$-continuity in the level $u$ for $J_{f}(u)$, $f$ being a general function that belongs to $\mathcal{B}_{1}$. In Section 3.2, first by using the coarea formula for an approximation of the functional, say $J_{f}(u, \sigma)(\sigma \rightarrow 0)$, we express this last one as stochastic integral with respect to Hermite polynomials. Then by using the $L^{2}$-continuity in the level for $J_{f}(u)$ we establish the $L^{2}$-convergence of $J_{f}(u, \sigma)$ towards $J_{f}(u)$, from which we deduce an Hermite expansion for the initial functional $J_{f}(u)$. As a derived product of the $L^{2}$-continuity in the level previously obtained we deduce in Section 3.3 a one order Rice formula for such functionals, in other words we compute $\mathbb{E}\left[J_{f}(u)\right]$.

In Section 4, the observation windows $\left(T_{n}\right)_{n}$ is a sequence of open bounded squares of $\mathbb{R}^{2}$, with the following form $\left.T_{n}:=\right]-n, n\left[^{2}\right.$ with $n \in \mathbb{N}^{\star}:=\{x \in \mathbb{Z}, x>0\}$, and $n$ tends to infinity. We focus on convergence results for $J_{f}^{(n)}(u)$. Section 4.1 is devoted to establish the almost sure convergence of $J_{f}^{(n)}(u)$ for general function $f$. In Section 4.2, by using the Hermite expansion of $J_{f}^{(n)}(u)$ derived from Section 3.2, we give the rate of this convergence. First the asymptotic variance as the squares $T_{n}$ grow to $\mathbb{R}^{2}$ is expressed as a series and we give an explicit lower bound. We then proved the asymptotic normality for the centred and suitably rescaled general functionals $J_{f}^{(n)}(u)$ through the Peccati-Tudor Theorem. The results obtained in Section 4 enable us, by considering in Section 5 the particular function $\overrightarrow{f^{\star}}$, to give rise to a first result, the definition of the estimators $\widehat{\lambda}_{n}$ and $\widehat{\theta}_{n}$ of the affinity parameters $\lambda$ and $\theta$. Secondly, we prove their almost sure consistency and also their rate of convergence in law in case where the true parameters live in an open set. Also, the coefficients of the asymptotic matrix variance are computed in Appendix A and a lower bound is given for its determinant. This law convergence result gives rise to confidence intervals for parameters $\lambda$ and $\theta$ in the specific special case where the covariance $r_{z}$ of the underlying isotropic process is known.

In Section 6 some complementary convergence results for $\widehat{\lambda}_{n}$ are proposed when the affinity parameters $\lambda$ and $\theta$ belong to the edges of the parameter space, including the particular case where $\lambda=1$. Finally, supposing that the covariance $r_{z}$ is known, we conclude by proposing an isotropy test.

This paper is complemented with Appendix A giving technical proofs of some lemmas.

\section{Notations and hypotheses}

\subsection{Notations}

Let us give some definitions, notations and some propositions and theorems related to properties of level sets and Rice formulas.

In the following $T$ is an open bounded rectangle of $\mathbb{R}^{2}$.

Let $(\Omega, \mathcal{A}, \mathcal{P})$ be a probability space and $X: \Omega \times T \subset \Omega \times \mathbb{R}^{2} \rightarrow \mathbb{R}$ a process continuously differentiable on $T$, that is $X \in \mathbf{C}^{1}(T)$.

Level sets We denote $D_{X}^{r}$ the following set

$$
\mathrm{D}_{\mathrm{X}}^{\mathrm{r}}:=\{t \in T: \nabla X(t) \text { is of rank } 1\}=\left\{t \in T:\|\nabla X(t)\|_{2} \neq 0\right\},
$$


where $\|\cdot\|_{2}$ denotes the Euclidean norm in $\mathbb{R}^{2}$, while $\nabla X$ stands for the Jacobian of $X$. For $u \in \mathbb{R}$ we define the level set at $u$ as:

$$
\mathcal{C}_{\mathrm{T}, \mathrm{X}}(u):=\{t \in T: X(t)=u\} .
$$

This set can be very irregular, but its intersection with $\mathrm{D}_{\mathrm{X}}^{\mathrm{r}}$, let $\mathcal{C}_{\mathrm{T}, \mathrm{X}}^{\mathrm{D}^{\mathrm{r}}}(u):=\mathcal{C}_{\mathrm{T}, \mathrm{X}}(u) \cap \mathrm{D}_{\mathrm{X}}^{\mathrm{r}}$ is a $\mathbf{C}^{1}$-manifold of dimension one. Indeed, at each point $t \in \mathcal{C}_{\mathrm{T}, \mathrm{X}}^{\mathrm{D}^{\mathrm{r}}}(u)$ the Jacobian matrix of $X$ is of full rank one. Thus, an application of the implicit theorem in a neighbourhood of each point $t$ provides a local parametrization of the level set $\mathcal{C}_{\mathrm{T}, \mathrm{X}}^{\mathrm{D}^{r}}(u)$. For more details concerning the proof of the construction of an atlas of $\mathcal{C}_{\mathrm{T}, \mathrm{X}}^{\mathrm{D}^{r}}(u)$, the reader can look at the beginning of the proof of [10, Theorem 3.1.2].

The following proposition (see Azaïs and Wschebor [8, Proposition 6.12]) gives a sufficient condition to ensure that the level is a regular value of $X$.

Proposition 2.1. Let $u \in \mathbb{R}^{2}$. We assume the following:

- The paths of $X$ are of class $\boldsymbol{C}^{2}$ on $T$.

- For each $t \in T$, the pair $(X(t), \nabla X(t))$ has a joint density $p_{X(t), \nabla X(t)}\left(x, x^{\prime}\right)$ in $\mathbb{R} \times \mathbb{R}^{2}$, which is bounded for $\left(t, x^{\prime}\right)$ varying in a compact subset of $T \times \mathbb{R}^{2}$ and $x$ in some neighbourhood of $u$.

Then

$$
\mathbb{P}\left(\omega \in \Omega, \exists t \in T, X(t)(\omega)=u,\|\nabla X(t)(\omega)\|_{2}=0\right)=0,
$$

that is almost surely

$$
\mathcal{C}_{T, X}^{D^{r}}(u)=\mathcal{C}_{T, X}(u)
$$

Remark 2.2. Note that if $X$ is a Gaussian process twice continuously differentiable on $T$, that is $X \in \mathbf{C}^{2}(T)$, such that for each $t \in T$, the vector $(X(t), \nabla X(t))$ has a joint density, then the last proposition hypotheses are verified and equality (2.1) holds. In that case the reader can also be referred to [10, Proposition 3.3.2].

General level functionals $S^{1}$ is the boundary of the unit ball of $\mathbb{R}^{2}$ and for $d=1,2$, $\sigma_{d}$ denotes the Lebesgue measure on $\mathbb{R}^{d}$. Recall that we defined $\mathcal{B}_{k}, k \in \mathbb{N}^{\star}$, the class of functions $f$ such that: $f: S^{1} \rightarrow \mathbb{R}^{k}$, is measurable, bounded, continuous except maybe on a finite number of points of the sphere $S^{1}$.

For $f: S^{1} \rightarrow \mathbb{R}$ belonging to $\mathcal{B}_{1}$, we define the following general functional $J_{f}(u)$ of the fixed level $u$ by:

$$
J_{f}(u):=\frac{1}{\sigma_{2}(T)} \int_{\mathcal{C}_{\mathrm{T}, \mathrm{X}}^{\mathrm{Dr}}(u)} f\left(\nu_{X}(t)\right) \mathrm{d} \sigma_{1}(t),
$$

where $\nu_{X}(t):=\frac{\nabla X(t)}{\|\nabla X(t)\|_{2}}$.

Remark 2.3. Note that for general process $X$, the finiteness of this integral is not necessarily guaranteed. However, this integral makes sense, for example, under assumptions of Proposition 2.1. More precisely, we substitute hypotheses concerning the process $X$ on $T$ in the last proposition by those on $O$, where $O$ is any open set such that $T \subset \bar{T} \subset O$, where $\bar{T}$ stands for the closure of $T$. In that case we deduce that almost surely $\mathcal{C}_{\overline{\mathrm{T}} \mathrm{X}}^{\mathrm{D}^{\mathrm{r}}}(u)=\mathcal{C}_{\overline{\mathrm{T}}, \mathrm{X}}(u)$. Then we construct a partition of unity for the compact manifold $\mathcal{C}_{\overline{\mathrm{T}}, \mathrm{X}}^{\mathrm{D}}(u)$ of $\mathbb{R}^{2}$, almost surely included in $\mathrm{D}_{\mathrm{O}, \mathrm{X}}^{\mathrm{r}}:=\left\{t \in O:\|\nabla X(t)\|_{2} \neq 0\right\}$. In that way as mentioned above, by using a local parametrization of the level set $\mathcal{C}_{\mathrm{T}, \mathrm{X}}^{\mathrm{D}^{\mathrm{r}}}(u)$, we prove that almost surely, $\sigma_{1}\left(\mathcal{C}_{\mathrm{T}, \mathrm{X}}^{\mathrm{\textrm {r }}}(u)\right)<+\infty$ (see also Remark 2.9). Since function $f$ is bounded, this construction guarantees the existence of functional $J_{f}(u)$. We also refer the reader to [10, Theorem 3.1.2] for such a partition of unity construction. 
Note that in this article, $T$ will increase to $\mathbb{R}^{2}$. In this regard, we will consider $T_{n}:=$ ]$-n, n\left[{ }^{2}\right.$, open squares of $\mathbb{R}^{2}$, with $n \in \mathbb{N}^{\star}$, a positive integer, and let $n$ tends to infinity. In this case, and with the comment made in Remark 2.3, the corresponding functional on the set $u$ will be denoted by $J_{f}^{(n)}(u)$, that is

$$
J_{f}^{(n)}(u):=\frac{1}{(2 n)^{2}} \int_{\mathcal{C}_{\mathrm{T}_{n}, \mathrm{X}}^{\mathrm{D}^{\mathrm{r}}}(u)} f\left(\nu_{X}(t)\right) \mathrm{d} \sigma_{1}(t) .
$$

Rice formulas The following results give Rice formulas for the first and second moments for general functionals defined on the level set of the process $X$ (see [8] or [10] for references).

By applying the coarea formula, for which a statement is expressed later in this text in Corollary 3.4, to general functionals defined on the level sets $\mathcal{C}_{\mathrm{T}, \mathrm{X}}^{\mathrm{\textrm {r }}}(u)$ and taking expectation afterwards, one obtains the well-known Kac-Rice formula. This formula gives a computation of the expectation of these general functionals for almost any level $u$. A proof of this formula is given for example in [10, Proposition 2.2.1]. Let us recall it here by adapting its statement to our context.

Let $\tilde{Y}: \Omega \times T \subset \Omega \times \mathbb{R}^{2} \rightarrow \mathbb{R}$ a process defined on $T$ and continuous on $\mathrm{D}_{\mathrm{X}}^{\mathrm{r}}$. Let us consider the following assumptions:

- $\mathbf{H}_{\mathbf{1}}$ : For almost any $t \in T$, the density of $X(t), \mathrm{p}_{X(t)}(\cdot)$ exists.

- $\mathbf{H}_{2}$ : The function

$$
u \longmapsto \mathbb{E}\left[\int_{\mathcal{C}_{\mathrm{T}, \mathrm{X}}^{\mathrm{D}}(u)}|\tilde{Y}(t)| \mathrm{d} \sigma_{1}(t)\right]
$$

is a continuous function of the variable $u$.

- $\mathbf{H}_{3}$ : The function

$$
u \longmapsto \int_{T} \mathrm{p}_{X(t)}(u) \mathbb{E}\left[|\widetilde{Y}(t)|\|\nabla X(t)\|_{2} \mid X(t)=u\right] \mathrm{d} t,
$$

is a continuous function of the variable $u$.

Proposition 2.2.1 of [10] implies the following proposition.

Proposition 2.4 (Kac-Rice formula). If $X$ and $\widetilde{Y}$ satisfy the assumption $\mathbf{H}_{\mathbf{1}}$ and $\left(\mathbf{H}_{\mathbf{2}}\right.$ or $\left.\mathbf{H}_{3}\right)$, then for almost any $u \in \mathbb{R}$ one has

$$
\mathbb{E}\left[\int_{\mathcal{C}_{T, X}^{D^{r}}(u)} \tilde{Y}(t) \mathrm{d} \sigma_{1}(t)\right]=\int_{T} p_{X(t)}(u) \mathbb{E}\left[\tilde{Y}(t)\|\nabla X(t)\|_{2} \mid X(t)=u\right] \mathrm{d} t .
$$

However, in applications the interest is focused on a fixed level $u$. So, we add the two following assumptions to ensure the validity of the Kac-Rice formula for all level.

- $\mathbf{H}_{4}$ : The function

$$
u \longmapsto \mathbb{E}\left[\int_{\mathcal{C}_{\mathrm{T}, \mathrm{X}}^{\mathrm{r}}(u)} \tilde{Y}(t) \mathrm{d} \sigma_{1}(t)\right],
$$

is a continuous function of the variable $u$.

- $\mathbf{H}_{5}$ : The function

$$
u \longmapsto \int_{T} \mathrm{p}_{X(t)}(u) \mathbb{E}\left[\tilde{Y}(t)\|\nabla X(t)\|_{2} \mid X(t)=u\right] \mathrm{d} t
$$

is a continuous function of the variable $u$. 
Adding the two previous assumptions, the two sides of equality (2.2) become now continuous as function of the level. Thus, we enunciate the following formula of Rice, for which a proof is given in [10, Theorem 3.1.1].

Theorem 2.5 (One order Rice formula). If $X$ and $\tilde{Y}$ satisfy the assumption $\mathbf{H}_{\mathbf{1}},\left(\mathbf{H}_{\mathbf{2}}\right.$ or $\left.\mathbf{H}_{\mathbf{3}}\right), \mathbf{H}_{\mathbf{4}}$ and $\mathbf{H}_{\mathbf{5}}$ then for any $u \in \mathbb{R}$ one has

$$
\mathbb{E}\left[\int_{\mathcal{C}_{T, X}^{D^{r}}(u)} \tilde{Y}(t) \mathrm{d} \sigma_{1}(t)\right]=\int_{T} p_{X(t)}(u) \mathbb{E}\left[\tilde{Y}(t)\|\nabla X(t)\|_{2} \mid X(t)=u\right] \mathrm{d} t .
$$

Remark 2.6. Note that Rice formula only requires for $\tilde{Y}$ to be continuous on $\mathrm{D}_{\mathrm{X}}^{\mathrm{r}}$, that will be the case for the process $\widetilde{Y}:=f\left(\nu_{X}\right) \mathbb{1}_{D_{\mathrm{X}}^{\mathrm{r}}}$ defined in the paragraph General level functionals, page 11, in case where $f$ is a continuous function on $S^{1}$.

Now we give conditions under which a two-order Rice formula will be valid. The reader is referred to [10, Theorem 3.3.3] adapted to the future situation.

Let us consider the following assumptions:

- $\mathbf{H}_{\mathbf{a}}: X: \Omega \times T \subset \Omega \times \mathbb{R}^{2} \rightarrow \mathbb{R}$ is a centred Gaussian random field twice continuously differentiable on $T$, that is $X \in \mathbf{C}^{2}(T)$.

- $\mathbf{H}_{\mathbf{b}}$ : For all $t \in T$, the vector $(X(t), \nabla X(t))$ has a density.

- $\mathbf{H}_{\mathbf{c}}$ For all $t_{1}, t_{2} \in T$, such that $t_{1} \neq t_{2}$, the vector $\left(X\left(t_{1}\right), X\left(t_{2}\right)\right)$ has a density, say $p_{X\left(t_{1}\right), X\left(t_{2}\right)}(\cdot, \cdot)$.

- $\mathbf{H}_{\mathbf{d}}$ :

$$
\mathbb{E}\left[\sup _{t \in T}\left\|\nabla^{2} X(t)\right\|_{1,2}^{(s)}\right]^{4}<+\infty
$$

where $\nabla^{2} X$ stands for the $2 \times 2$ Hessian matrix of $X$ and $\|\cdot\|_{1,2}^{(s)}$ stands for the norm in $\mathfrak{L}_{\mathfrak{s}}^{2}\left(\mathbb{R}^{2}, \mathbb{R}\right)$, the vectorial space of symmetric linear continuous functions from $\mathbb{R}^{2}$ to $\mathbb{R}$.

- $\mathbf{H}_{\mathbf{e}}: Y: \Omega \times T \subset \Omega \times \mathbb{R}^{2} \rightarrow \mathbb{R}$ is a continuous process defined on $T$ as $Y(t):=$ $G(t, \nabla X(t))$, where

$$
\begin{aligned}
G: T \times \mathbb{R}^{2} & \longrightarrow \mathbb{R} \\
(t, z) & \mapsto G(t, z),
\end{aligned}
$$

is a bounded continuous function on $T \times \mathbb{R}^{2}$.

Let us now state the following assumption $\mathbf{H}_{6}$.

- $\mathbf{H}_{6}$ : For all $u \in \mathbb{R}$,

$$
\begin{aligned}
\int_{T \times T} \mathbb{E}\left[\left|Y\left(t_{1}\right)\right|\left|Y\left(t_{2}\right)\right|\left\|\nabla X\left(t_{1}\right)\right\|_{2}\left\|\nabla X\left(t_{2}\right)\right\|_{2}\right. & \left.\mid X\left(t_{1}\right)=X\left(t_{2}\right)=u\right] \\
& \times p_{X\left(t_{1}\right), X\left(t_{2}\right)}(u, u) \mathrm{d} t_{1} \mathrm{~d} t_{2}<+\infty .
\end{aligned}
$$

Let us express the following two-order Rice formula.

Theorem 2.7 (Two-order Rice formula). If $X$ and $Y$ satisfy the assumptions $\mathbf{H}_{\mathbf{a}}, \mathbf{H}_{\mathbf{b}}, \mathbf{H}_{\mathbf{c}}$, $\mathbf{H}_{\mathbf{d}}, \mathbf{H}_{\mathbf{e}}$ and $\mathbf{H}_{6}$, then for any $u \in \mathbb{R}$ one has

$$
\begin{gathered}
\mathbb{E}\left[\int_{\mathcal{C}_{T, X}^{D^{r}}(u)} Y(t) \mathrm{d} \sigma_{1}(t)\right]^{2} \\
=\int_{T \times T} \mathbb{E}\left[Y\left(t_{1}\right) Y\left(t_{2}\right)\left\|\nabla X\left(t_{1}\right)\right\|_{2}\left\|\nabla X\left(t_{2}\right)\right\|_{2} \mid X\left(t_{1}\right)=X\left(t_{2}\right)=u\right] \times \\
p_{X\left(t_{1}\right), X\left(t_{2}\right)}(u, u) \mathrm{d} t_{1} \mathrm{~d} t_{2} .
\end{gathered}
$$


From this theorem we deduce the following corollary.

Corollary 2.8. Let $X: \Omega \times \mathbb{R}^{2} \rightarrow \mathbb{R}$ be a centred stationary Gaussian random field twice continuously differentiable on $\mathbb{R}^{2}$, that is $X \in \boldsymbol{C}^{2}\left(\mathbb{R}^{2}\right)$, with covariance $r_{x}$ (belonging necessarily to $\boldsymbol{C}^{4}\left(\mathbb{R}^{2}\right)$ ) satisfying the following hypotheses:

- $\nabla^{2} r_{x}(0)$ is a non-degenerate matrix, where $\nabla^{2} r_{x}$ stands for the $2 \times 2$ Hessian matrix of $r_{x}$.

- $r_{x}^{2}(0)-r_{x}^{2}(t) \neq 0$, for all $t \neq 0$.

Then assumptions $\mathbf{H}_{\mathbf{a}}, \mathbf{H}_{\mathbf{b}}, \mathbf{H}_{\mathbf{c}}$ and $\mathbf{H}_{\mathbf{d}}$ are checked so that for all $u \in \mathbb{R}$, almost surely

$$
\mathcal{C}_{T, X}^{D^{r}}(u)=\mathcal{C}_{T, X}(u) .
$$

Furthermore if $X$ and $Y$ satisfy the assumption $\mathbf{H}_{\mathbf{e}}$ then they satisfy also assumptions $\mathbf{H}_{6}$. Thus, for any $u \in \mathbb{R}$ the two-order Rice formula (2.4) is valid.

The proof of this corollary provides from the following argumentation.

Hypotheses made on $X$, ensure that $X$ is a Gaussian process twice continuously differentiable on $T$, such that for each $t \in T$ the vector $(X(t), \nabla X(t))$ has a joint density, since $X(t)$ and $\nabla X(t)$ are independent random variables. Thus Remark 2.2 gives equality (2.1). Furthermore, these hypotheses ensure that assumption $\mathbf{H}_{\mathbf{c}}$ is obviously checked. By using the Borel-TIS inequality given in Adler and Taylor [2, Theorem 2.1.1], one can show that assumption $\mathbf{H}_{\mathbf{d}}$ is also checked.

Now let $X$ and $Y$ satisfying the assumption $\mathbf{H}_{\mathbf{e}}$. The fact that $G$ is a bounded function on $T \times \mathbb{R}^{2}$, implies that $Y$ is a bounded process on $T$. Thus, to check assumption $\mathbf{H}_{6}$, it is enough to establish that for all $u \in \mathbb{R}$, one has

$$
\int_{T \times T} \mathbb{E}\left[\left\|\nabla X\left(t_{1}\right)\right\|_{2}\left\|\nabla X\left(t_{2}\right)\right\|_{2} \mid X\left(t_{1}\right)=X\left(t_{2}\right)=u\right] \times p_{X\left(t_{1}\right), X\left(t_{2}\right)}(u, u) \mathrm{d} t_{1} \mathrm{~d} t_{2}<+\infty .
$$

The proof of this finiteness can be found for example in Berzin and Wschebor [11, Proposition 2].

Thus $X$ and $Y$ satisfy assumptions given in Theorem 2.7 and the two-order Rice formula ensues from it.

Remark 2.9. One can observe that under the hypotheses of Corollary 2.8 for $X$, by taking $Y \equiv 1$, one has proved that for any $u \in \mathbb{R}$,

$$
\begin{aligned}
& \mathbb{E}\left[\sigma_{1}\left(\mathcal{C}_{\mathrm{T}, \mathrm{X}}^{\mathrm{D}^{\mathrm{r}}}(u)\right)\right]^{2}= \\
& \int_{T \times T} \mathbb{E}\left[\left\|\nabla X\left(t_{1}\right)\right\|_{2}\left\|\nabla X\left(t_{2}\right)\right\|_{2} \mid X\left(t_{1}\right)=X\left(t_{2}\right)=u\right] \times p_{X\left(t_{1}\right), X\left(t_{2}\right)}(u, u) \mathrm{d} t_{1} \mathrm{~d} t_{2}<+\infty .
\end{aligned}
$$

Note that in that case we proved that almost surely $\mathcal{C}_{\mathrm{T}, \mathrm{X}}^{\mathrm{D}}(u)<+\infty$ (resp. for all $n \in \mathbb{N}^{\star}$, $\left.\mathcal{C}_{\mathrm{T}_{n}, \mathrm{X}}^{\mathrm{r}}(u)<+\infty\right)$. Thus, for $f: S^{1} \rightarrow \mathbb{R}$ a measurable and bounded function, the functional $J_{f}(u)$ (resp. $J_{f}^{(n)}(u)$ ) is almost surely well defined.

Also note that under the hypotheses of Corollary 2.8 for $X$, those slightly modified of Proposition 2.1 are also checked (see Remark 2.3). If we proceed as indicated in the previous remark, we then obtain another way to establish the almost sure finiteness of $\mathcal{C}_{\mathrm{T}, \mathrm{X}}^{\mathrm{r}}(u)$ and also that of $\mathcal{C}_{\mathrm{T}_{n}, \mathrm{X}}^{\mathrm{D}^{\mathrm{r}}}(u)$. This explicit way provides another manner for proving the almost sure existence of functional $J_{f}(u)$ (resp. $J_{f}^{(n)}(u)$ ).

Remark 2.10. Note that process $\widetilde{Y}:=f\left(\nu_{X}\right) \mathbb{1}_{\mathrm{D}_{\mathrm{X}}^{\mathrm{r}}}$ defined in the paragraph General level functionals is such that processes $X$ and $\widetilde{Y}$ do not verify hypothesis $\mathbf{H}_{\mathbf{e}}$, since $\widetilde{Y}$ is in the best way continuous on $\mathrm{D}_{\mathrm{X}}^{\mathrm{r}}$ and a priori not continuous on $T$. So, to compute $\mathbb{E}\left[J_{f}(u)\right]^{2}$ we will have to play close to the vest and be creative by generalizing the two order Rice formula to our purpose (see forthcoming Lemma 3.2). 
Isotropic process A process $\left\{Z(t), t \in \mathbb{R}^{2}\right\}$ is said to be isotropic if it is a stationary process and if for any isometry $J$ in $\mathbb{R}^{2}, k \in \mathbb{N}:=\{x \in \mathbb{Z}, x \geqslant 0\}$ and $t_{1}, \ldots, t_{k} \in \mathbb{R}^{2}$, the joint laws of $\left(Z\left(t_{1}\right), \ldots, Z\left(t_{k}\right)\right)$ and $\left(Z\left(J . t_{1}\right), \ldots, Z\left(J . t_{k}\right)\right)$ are the same.

Affine process A process $\left\{X(t), t \in \mathbb{R}^{2}\right\}$ is said to be an affine process when it is equal in law to $\left\{Z(A . t), t \in \mathbb{R}^{2}\right\}$, where $Z$ is isotropic and $A: \mathbb{R}^{2} \rightarrow \mathbb{R}^{2}$ is a linear self-adjoint transformation.

If the eigenvalues of $A$ are denoted by $\lambda_{1}, \lambda_{2}$, saying that the process $X$ is isotropic means that $\lambda_{1}=\lambda_{2}$ (see Adler and Taylor [2, Section 5.7]).

Hermite polynomials We use the Hermite polynomials $\left(H_{q}\right)_{q \in \mathbb{N}}$ defined by

$$
H_{q}(x):=(-1)^{q} \phi^{-1}(x) \frac{\mathrm{d}^{q}}{\mathrm{~d} x^{q}}(\phi(x)),
$$

where $\phi$ denotes the standard Gaussian density on $\mathbb{R}$.

They provide an orthogonal basis of $L^{2}(\mathbb{R}, \phi(x) \mathrm{d} x)$. We also denote by $\phi_{m}$ the standard Gaussian density on $\mathbb{R}^{m}$, for $m=2$ or 3 .

For $\boldsymbol{k}:=\left(k_{1}, k_{2}, k_{3}\right) \in \mathbb{N}^{3}$ and $y:=\left(y_{1}, y_{2}, y_{3}\right) \in \mathbb{R}^{3}$, we set $|\boldsymbol{k}|:=k_{1}+k_{2}+k_{3}$, $\boldsymbol{k} !:=k_{1} ! k_{2} ! k_{3}$ ! and $\widetilde{H}_{\boldsymbol{k}}(y):=\prod_{1 \leqslant j \leqslant 3} H_{k_{j}}\left(y_{j}\right)$.

Asymptotic equivalence symbol $\simeq$ For a set of random variables $X_{n}$, the notation $X_{n}=o_{P}(1)$ means that $X_{n}$ converges to zero in probability when $n$ goes to infinity. Furthermore, if $Y_{n}$ is a set of random variables such that $X_{n}=Y_{n}+o_{P}(1)$, we will note $X_{n} \simeq Y_{n}$.

We denote by $\langle\cdot, \cdot\rangle$ the canonical scalar product in $\mathbb{R}^{2}$.

$\mathbf{C}$ is a generic constant that could change value while developing a proof.

\subsection{Hypotheses}

Let $(\Omega, \mathcal{A}, \mathcal{P})$ be a probability space and $X: \Omega \times \mathbb{R}^{2} \rightarrow \mathbb{R}$ an affine process, equal in law to $\left\{Z(A . t), t \in \mathbb{R}^{2}\right\}$, where $Z: \Omega \times \mathbb{R}^{2} \rightarrow \mathbb{R}$ is isotropic and $A: \mathbb{R}^{2} \rightarrow \mathbb{R}^{2}$ is a linear self-adjoint transformation. We additionally suppose that $A$ is a positive definite transformation.

We also assume that $Z$ is a centred Gaussian process twice continuously differentiable in $\mathbb{R}^{2}$, that is $Z \in \mathbf{C}^{2}\left(\mathbb{R}^{2}\right)$.

The eigenvalues of $A$ are denoted by $\lambda_{1}, \lambda_{2}, 0<\lambda_{2} \leqslant \lambda_{1}$. Let $0<\lambda \leqslant 1$ be the quotient of the eigenvalues, $\lambda:=\lambda_{2} / \lambda_{1}$. The process $X$ is isotropic means that $\lambda=1$ (see Adler and Taylor [2, Section 5.7]).

Let $r_{z}$ and $r_{x}$ be the covariance function of $Z$ and $X$ respectively. The regularity assumption on $Z$ implies that $r_{z} \in \mathbf{C}^{4}\left(\mathbb{R}^{2}\right)$.

Let $T$ be an open bounded rectangle of $\mathbb{R}^{2}$. Let also $\left(T_{n}\right)_{n}$ be open bounded squares with the following form $\left.T_{n}:=\right]-n, n\left[^{2}\right.$ with $n \in \mathbb{N}^{\star}$, and let $n$ tends to infinity.

Assumptions on the covariance For any multidimensional index $\boldsymbol{m}:=\left(i_{1}, \ldots, i_{k}\right)$ with $1 \leqslant k \leqslant 2$ and $1 \leqslant i_{j} \leqslant 2$, we write $\frac{\partial^{m} r_{z}}{\partial t^{m}}(t):=\frac{\partial^{k} r_{z}}{\partial t_{i_{1}} \cdots \partial t_{i_{k}}}(t)$. Let

$$
\Psi(t):=\max \left\{\left|r_{z}(t)\right|,\left|\frac{\partial^{\boldsymbol{m}} r_{z}}{\partial t^{\boldsymbol{m}}}(t)\right|, \boldsymbol{m} \in\{1,2\}^{k}, 1 \leqslant k \leqslant 2\right\} .
$$

We make the following assumption. 
- $\mathbf{H}_{\Psi}: \Psi(t) \rightarrow 0$ when $\|t\|_{2} \rightarrow+\infty, \Psi \in L^{1}\left(\mathbb{R}^{2}\right)$ and $\int_{\mathbb{R}^{2}} r_{z}(t) \mathrm{d} t>0$.

Remark 2.11. Note that $r_{z} \in L^{1}\left(\mathbb{R}^{2}\right)$ implies that $r_{z} \in L^{q}\left(\mathbb{R}^{2}\right)$ for all $q \geqslant 1$ and hence that $Z$ (resp. X) admits a spectral density $f_{z}$ (resp. $f_{x}$ ) that is continuous and such that $f_{z}(0)>0$. We wish to emphasize that this fact implies that the following assumption $\mathbf{H}_{\mathbf{r}}$ is always fulfilled

- $\mathbf{H}_{\mathbf{r}}: r_{z}^{2}(0)-r_{z}^{2}(t) \neq 0$, for all $t \neq 0$.

Remark 2.12. Note also that $\int_{\mathbb{R}^{2}} f_{z}(t)\|t\|_{2}^{2} \mathrm{~d} t<+\infty$.

Comments on assumptions made Having the property that $f_{z}(0)>0$ ensures that $\nabla^{2} r_{x}(0)$ is a non-degenerate matrix. Indeed $-\nabla^{2} r_{x}(0)=\mu A^{2}$, where $\mu:=-\frac{\partial^{2} r_{z}}{\partial t_{i}^{2}}(0)=$ $\frac{1}{2} \int_{\mathbb{R}^{2}} f_{z}(t)\|t\|_{2}^{2} \mathrm{~d} t$, for any $i=1,2$. Thus $\mu>0$.

Hypothesis $\mathbf{H}_{\mathbf{r}}$ being fulfilled ensures that the assumptions of Corollary 2.8 are verified, so that its conclusions are also verified. That is for all $u \in \mathbb{R}$, equality (2.1) is almost surely valid (for $T$ or $T_{n}$ ). Furthermore if $f: S^{1} \rightarrow \mathbb{R}$ is a measurable and bounded function, functionals $J_{f}(u)$ and $J_{f}^{(n)}(u)\left(n \in \mathbb{N}^{\star}\right)$ are almost surely well defined (see Remark 2.9). Also if $X$ and $Y$ satisfies the assumption $\mathbf{H}_{\mathbf{e}}$ with $T$ or $T_{n}$ then the twoorder Rice formula (2.4) is valid for $T$ or $T_{n}$.

Remark 2.13. By using Proposition 6.11 in Azaïs and Wschebor [8], one can also show that for all $u \in \mathbb{R}$ and for any fixed point $x$ belonging to the sphere $S^{1}$,

$$
\mathbb{P}\left(\omega \in \Omega, \exists t \in T, X(t)(\omega)=u, \nu_{X}(t)(\omega)=x\right)=0 .
$$

Last equality still remains valid for $T_{n}$.

A sufficient element of proving is establishing that the result is true for the isotropic process $Z$. This result follows from the fact that the vector $\nu_{Z}(0):=\frac{\nabla Z(0)}{\|\nabla Z(0)\|_{2}}$ and $Z(0)$ are independent and that the distribution of $\nu_{Z}(0)$ is uniform on $S^{1}$ (see proposition given in Wschebor [32], page 79).

The assumption $\mathbf{H}_{\Psi}$ will be useful to prove Proposition 4.3 in which we exhibit the asymptotic variance of the centred and suitably rescaled general functionals $J_{f}^{(n)}(u)$ as $n$ tends to infinity. The fact that $f_{z}(0)>0$ ensures that this limit variance is not trivial.

These comments lead us to adopt the following new notations.

Notations We shall omit in the future the symbol $\mathrm{D}^{\mathrm{r}}$. Furthermore for writing convenience if $u$ is a fixed level belonging to $\mathbb{R}$, we will note $\mathcal{C}(u)$ in place of $\mathcal{C}_{\mathrm{T}, \mathrm{X}}^{\mathrm{D}}(u)$ and $\mathcal{C}_{n}(u)$ in place of $\mathcal{C}_{\mathrm{T}_{n}, \mathrm{X}}^{\mathrm{D}^{\mathrm{r}}}(u)$. Also, for $B$ a rectangle set included in $T_{n}$, we will note $\mathcal{C}_{B}(u)$ in place of $\mathcal{C}_{\mathrm{B}, \mathrm{X}}^{\mathrm{r}}(u)$.

Particular isotropic processes Here we give three examples of isotropic processes satisfying assumption $\mathbf{H}_{\Psi}$.

Since $Z$ is a stationary Gaussian process, $Z$ will be isotropic if, and only if, its covariance $r_{z}$ will only depend on the norm. Furthermore $Z$ must have a spectral density $f_{z}$, so this last condition is equivalent to the one that $f_{z}$ will depend only on the norm.

Powered exponential covariance The first example is given by a covariance function belonging to the class of powered exponential family (see Yaglom [33, Chap. 4.22.2, example 3, p. 364]), that is: $r_{z}(t):=C \exp \left(-\alpha\|t\|_{2}^{2}\right), t \in \mathbb{R}^{2}, C>0, \alpha>0$ being a scale parameter. One has, $\int_{\mathbb{R}^{2}} r_{z}(t) \mathrm{d} t=\frac{C \pi}{\alpha}>0$. Here the spectral density $f_{z}$ is $f_{z}(\lambda):=\frac{C}{4 \pi \alpha} \exp \left(-\frac{1}{4 \alpha}\|\lambda\|_{2}^{2}\right), \lambda \in \mathbb{R}^{2}$. The covariance $r_{z}$ satisfies assumption $\mathbf{H}_{\Psi}$. 
Generalized Cauchy covariance The second example of covariance function is given by the one belonging to the Cauchy family (see [33, Chap. 4.22.2, example 5, p. 365], see also Anderson [6] and Lim and Teo [21, equality (2.1) p. 1326]), that is $r_{z}(t):=$ $C\left(\alpha^{2}+\|t\|_{2}^{2}\right)^{-\nu}, t \in \mathbb{R}^{2}, C>0, \alpha>0$ being a scale parameter and $\nu>0$ a smoothness parameter controlling the long range dependence of the process $Z$. The spectral density is

$$
f_{z}(\lambda):=\frac{C \alpha^{1-\nu}\|\lambda\|_{2}^{\nu-1}}{2^{\nu} \pi \Gamma(\nu)} K_{\nu-1}\left(\alpha\|\lambda\|_{2}\right)
$$

(see Yaglom [34, Chap. 4, equality (4.12'), p. 129], see also Lim and Teo [22, p. 3]). Here $K_{\nu}$ is the modified Bessel function of the third kind (see Gradshteyn and Ryzhik [18, 8.432 p. 917] for definition) and $\Gamma$ is the Gamma function defined in Appendix (A.5).

If we add the condition $\nu>1$, then $\int_{\mathbb{R}^{2}} r_{z}(t) \mathrm{d} t=\frac{C \pi \alpha^{2-2 \nu}}{\nu-1}>0$, and covariance $r_{z}$ satisfies assumption $\mathbf{H}_{\Psi}$.

Whittle-Matérn covariance The third example of covariance function is given by the one belonging to the Matérn class (see [33, Chap. 4.22.2, example 2, p. 363], see also [22, equality (1) p. 2]). The covariance function is given by $r_{z}(t):=C\left(\alpha\|t\|_{2}\right)^{\nu} K_{\nu}\left(\alpha\|t\|_{2}\right)$, $t \in \mathbb{R}^{2}, C>0, \alpha>0$ being a scale parameter controlling the spatial range of the covariance and $\nu>0$ is the smoothness parameter governing the level of smoothness of $Z(t)$. The spectral density is $f_{z}(\lambda):=\frac{C 2^{\nu-1} \Gamma(\nu+1) \alpha^{2 \nu}}{\pi\left(\alpha^{2}+\|\lambda\|_{2}^{2}\right)^{\nu+1}}$ (see [33, equality (4.131) p. 364, Chap. 4.22.2, example 2] and also [21, Proposition 3.1 p. 1333]). Since $\nu>-1$, using the spectral density representation of the covariance in zero in the second example one can prove that $\int_{\mathbb{R}^{2}} r_{z}(t) \mathrm{d} t=C \pi \alpha^{-2} 2^{\nu+1} \Gamma(\nu+1)>0$. In addition, if we suppose that $\nu>2$, one can easily show that $\int_{\mathbb{R}^{2}}\|\lambda\|_{2}^{4} f(\lambda) d \lambda<+\infty$, so that the covariance function $r_{z} \in \mathbf{C}^{4}\left(\mathbb{R}^{2}\right)$ (one can also refer to [22, (33) and (34), p. 9]). By using [18, equalities 10 and 11 of $8.486(1)$ p. 929] we obtain the expression of the derivatives of function $r_{z}$. In [18], equalities 8.485 p. 928 and 8.445 and 8.446 p. 919 give the asymptotic behaviour of $K_{\nu}(z)$ when $z \rightarrow 0$ and 6 . of 8.451 p. 920 the one when $z \rightarrow+\infty$. With these properties one can establish in case where $\nu>2$ that $\Psi(t) \rightarrow 0$ when $\|t\|_{2} \rightarrow+\infty$ and that $\Psi \in L^{1}\left(\mathbb{R}^{2}\right)$. In conclusion we deduce that when $\nu>2$ the covariance $r_{z}$ satisfies assumption $\mathbf{H}_{\Psi}$.

Commentaries about these examples In the two first examples we remark that covariance $r_{z}$ is strictly decreasing as function of the distance from the origin. If the linear transformation $A$ is not an isometry, this property vanishes since points living in a circle centred at the origin with same covariance $r_{z}$ will be transformed by $A$ into points over an ellipse and thus with different covariances $r_{x}$. In other words and in a general way, meaning not just for the two previous examples, circles are deformed by stretching via the transformation $A$. A point $t \in \mathbb{R}^{2}$ such that $\|t\|_{2}=1$, that is living in the unity circle, will be such that $A \cdot t$ will belong to the centred ellipse at the origin defined by the $A$-eigenvectors directions $\left(v_{1}, v_{2}\right)$ as axis and by the eigenvalues $\lambda_{1}, \lambda_{2}$ the magnitude of the axis. Measuring the range of this stretching will be part of the matter of the paper, through the construction of estimators of the ratio of the eigenvalues, say $\lambda=\lambda_{2} / \lambda_{1}$, and of $\theta$, the angle rotation between the eigenvectors $\left(v_{1}, v_{2}\right)$ and a fixed direct orthonormal basis $\left(v^{\star}, v^{\star \star}\right)$ (see Figure 1 page 40 ).

\section{Hermite expansion for level functionals}

In this section $T$ will be a fixed open bounded rectangle of $\mathbb{R}^{2}$. Assumptions on $X$ are the same as in Section 2.2.

For a function $f: S^{1} \rightarrow \mathbb{R}$, that belongs to $\mathcal{B}_{1}$, recall that we have defined in Section 2.1 
the following general functional $J_{f}(u)$ of the level $u$ as:

$$
J_{f}(u):=\frac{1}{\sigma_{2}(T)} \int_{\mathcal{C}(u)} f\left(\nu_{X}(t)\right) \mathrm{d} \sigma_{1}(t),
$$

where $\nu_{X}(t):=\frac{\nabla X(t)}{\|\nabla X(t)\|_{2}}$.

Our objective is for $u$ a fixed level to give an Hermite expansion of the random variable $J_{f}(u)$.

In this aim the idea consists in approaching functionals $J_{f}(u)$ by other functionals, say $J_{f}(u, \sigma)(\sigma \rightarrow 0)$, in such a way that the last ones can be expressed as stochastic integrals with respect to Hermite polynomials. This is possible through the use of a kernel $K_{\sigma}$ and via the coarea formula. Then the idea will consist in proving that $J_{f}(u, \sigma)$ tends in $L^{2}(\Omega)$ towards $J_{f}(u)$ as $\sigma \rightarrow 0$.

We emphasize that the convergence is not an obvious purpose since the difficulty is to prove the continuity of function $\left(u_{1}, u_{2}\right) \mapsto \mathbb{E}\left[J_{f}\left(u_{1}\right) J_{f}\left(u_{2}\right)\right]$ that will be the aim of the next paragraph. This fact is far from being trivial and requires a number of results stated in Section 2.1, as the second order Rice formula, and also studied in next section, as a in-depth study of the level curve $\mathcal{C}(u+\delta)$ in a neighbourhood of the level $u$.

By using the $L^{2}$-continuity in the level $u$ of functional $J_{f}(u)$, we will obtain as by product an expression for its first moment, that is a first order Rice formula for all level.

\section{1 $L^{2}$-continuity in the level}

We prove the following theorem.

Theorem 3.1. Let $f: S^{1} \rightarrow \mathbb{R}$ be a function that belongs to $\mathcal{B}_{1}$. Then the function

$$
\left(u_{1}, u_{2}\right) \mapsto \mathbb{E}\left[J_{f}\left(u_{1}\right) J_{f}\left(u_{2}\right)\right],
$$

is continuous. In particular the same holds for function,

$$
u \mapsto \mathbb{E}\left[J_{f}(u)\right]^{2} .
$$

Proof of Theorem 3.1. As explained in Remark 2.10, process $\widetilde{Y}:=f\left(\nu_{X}\right) \mathbb{1}_{\mathrm{D}_{\mathrm{x}}^{\mathrm{r}}}$ is not continuous on $T$, so we can not apply Corollary 2.8 to compute $\mathbb{E}\left[J_{f}(u)\right]^{2}$. We need to be a little careful. In fact the following lemma will prove as by product that such computation holds for $\mathbb{E}\left[J_{f}(u)\right]^{2}$ and that function $u \mapsto \mathbb{E}\left[J_{f}(u)\right]^{2}$ is continuous.

Let us state this lemma proved in Appendix and for which the proof is based on the application of Corollary 2.8.

Let $H: T \times\left(\mathbb{R}^{2}\right)^{\star} \times S^{1}$ be a measurable bounded continuous function of its arguments except maybe on $T \times\left(\mathbb{R}^{2}\right)^{\star} \times \cup_{i=1}^{\ell}\left\{x_{i}\right\}$, where $x_{i}, i=1, \ell$, are a finite number of fixed points of the sphere $S^{1}$. We have noted $\left(\mathbb{R}^{2}\right)^{\star}:=\left\{v \in \mathbb{R}^{2},\|v\|_{2} \neq 0\right\}$.

Lemma 3.2. Let $F: T \times\left(\mathbb{R}^{2}\right)^{\star} \rightarrow \mathbb{R}$ defined by $F(t, z):=H\left(t, z, \frac{z}{\|z\|_{2}}\right)$, then

$$
u \mapsto \mathbb{E}\left[\int_{\mathcal{C}(u)} F(t, \nabla X(t)) \mathbb{1}_{D_{X}^{r}}(t) \mathrm{d} \sigma_{1}(t)\right]^{2}
$$

is a continuous function.

Furthermore, for any $u \in \mathbb{R}$ one has

$$
\begin{aligned}
& \mathbb{E}\left[\int_{\mathcal{C}(u)} F(t, \nabla X(t)) \mathbb{1}_{D_{X}^{r}}(t) \mathrm{d} \sigma_{1}(t)\right]^{2} \\
& =\int_{T \times T} \mathbb{E}\left[F\left(t_{1}, \nabla X\left(t_{1}\right)\right) \mathbb{1}_{D_{X}^{r}}\left(t_{1}\right) F\left(t_{2}, \nabla X\left(t_{2}\right)\right) \mathbb{1}_{D_{X}^{r}}\left(t_{2}\right)\right. \\
& \left.\quad \times\left\|\nabla X\left(t_{1}\right)\right\|_{2}\left\|\nabla X\left(t_{2}\right)\right\|_{2} \mid X\left(t_{1}\right)=X\left(t_{2}\right)=u\right] p_{X\left(t_{1}\right), X\left(t_{2}\right)}(u, u) \mathrm{d} t_{1} \mathrm{~d} t_{2},
\end{aligned}
$$

where $p_{X\left(t_{1}\right), X\left(t_{2}\right)}(u, u)$ stands for the density of $\left(X\left(t_{1}\right), X\left(t_{2}\right)\right)$ in point $(u, u)$. 
Let $u$ be fixed in $\mathbb{R}$ and $\left(u_{k}\right)_{k \in \mathbb{N}}$ be a real sequence tending to $u$. Let us note $I_{f}(u):=\int_{\mathcal{C}(u)} f\left(\nu_{X}(t)\right) \mathrm{d} \sigma_{1}(t)$.

Since $f \in \mathcal{B}_{1}$, let $\left\{x_{1}, \cdots, x_{\ell}\right\}, \ell \in \mathbb{N}^{\star}$, the finite set of the points of the sphere $S^{1}$, where $f$ is discontinuous. By Remark 2.13, almost surely

$$
I_{f}(u)=\int_{\mathcal{C}(u)} f\left(\nu_{X}(t)\right) \prod_{i=1}^{\ell} \mathbb{1}_{\left\{\nu_{X}(t) \neq x_{i}\right\}} \mathrm{d} \sigma_{1}(t) .
$$

Proving Theorem 3.1 is equivalent to show that $\left\|I_{f}\left(u_{k}\right)-I_{f}(u)\right\|_{L^{2}(\Omega)}$ tends to zero as $k$ goes to infinity, where $\|\cdot\|_{L^{2}(\Omega)}$ stands for the $L^{2}(\Omega)$-norm.

Thus we try to apply the Scheffé's lemma but it is not so direct. Indeed Lemma 3.2 applied to function $F: T \times\left(\mathbb{R}^{2}\right)^{\star} \rightarrow \mathbb{R}$, such that

$$
F(t, z):=f\left(z /\|z\|_{2}\right) \prod_{i=1}^{\ell} \mathbb{1}_{\left\{\left(z /\|z\|_{2}\right) \neq x_{i}\right\}}
$$

implies that $\left\|I_{f}\left(u_{k}\right)\right\|_{L^{2}(\Omega)}$ tends to $\left\|I_{f}(u)\right\|_{L^{2}(\Omega)}$ as $k$ goes to infinity. But we need the missing following convergence, that is, almost surely $I_{f}\left(u_{k}\right)$ tends to $I_{f}(u)$, with $k$.

Our goal is now to tempt to apply the following theorem stated and proved as [10, Theorem 3.1.2]. Note that in its statement the functions of interest are deterministic, that is they are not random functions.

Theorem 3.3. Let $g: T \subset \mathbb{R}^{2} \rightarrow \mathbb{R}$ be a function belonging to $\boldsymbol{C}^{1}(T)$ such that $\nabla g$ is Lipschitz on $T$. Let $h: T \subset \mathbb{R}^{2} \rightarrow \mathbb{R}$ be a continuous function on $T$ such that $\operatorname{supp}(h) \subset D_{g}^{r}:=\left\{t \in T:\|\nabla g(t)\|_{2} \neq 0\right\}, \operatorname{supp}(h)$ being the support of function $h$. Then the function

$$
u \rightarrow \int_{\mathcal{C}_{T, g}^{D^{r}}(u)} h(t) \mathrm{d} \sigma_{1}(t)
$$

is continous with respect to the variable $u$. We have noted $\mathcal{C}_{T, g}^{D^{r}}(u):=\left\{t \in T: g(t)=u,\|\nabla g(t)\|_{2} \neq 0\right\}$.

Remark that process $X$ is such that $\nabla X$ is almost surely Lipschitz on $T$. Indeed we have already mentioned after the statement of Corollary 2.8 that assumption $\mathbf{H}_{\mathbf{d}}$ remains checked. Thus equality (2.3) is valid, that is $\mathbb{E}\left[\sup _{t \in T}\left\|\nabla^{2} X(t)\right\|_{1,2}^{(s)}\right]^{4}<+\infty$. So we can conclude that almost surely $L_{X}:=\sup _{t \in T}\left\|\nabla^{2} X(t)\right\|_{1,2}^{(s)}<+\infty$. Furthermore the Taylor formula applied to $X$ belonging to $\mathbf{C}^{2}(T)$ ensures that almost surely $\nabla X$ is Lipschitz with Lipschitz constant $L_{X}$.

As already mentioned in Section 2.1 process $\widetilde{Y}:=f\left(\nu_{X}\right) \mathbb{1}_{\mathrm{D}_{\mathrm{X}}^{\mathrm{r}}}$ is not continuous on $T$ and there is no reason for why $\operatorname{supp}(\tilde{Y}) \subset \mathrm{D}_{\mathrm{X}}^{\mathrm{r}}$.

In view of applying the Scheffé's lemma the idea consists in approximating the functional $I_{f}(u)$ by the following one. It will check hypotheses of Theorem 3.3 and also that of Lemma 3.2. For $m \in \mathbb{N}^{\star}$, let

$$
I_{f, m}(u):=\int_{\mathcal{C}(u)} Y_{m}(t) \mathrm{d} \sigma_{1}(t)
$$

where we define for $t \in T$

$$
Y_{m}(t):=F_{m}(t, \nabla X(t)) \mathbb{1}_{\mathrm{D}_{\mathrm{X}}^{\mathrm{r}}}(t),
$$

with function $F_{m}: T \times\left(\mathbb{R}^{2}\right)^{\star} \rightarrow \mathbb{R}$, defined by

$$
F_{m}(t, z):=g_{m}(t) \varphi\left(\frac{1}{m\|z\|_{2}}\right) f\left(z /\|z\|_{2}\right) \prod_{i=1}^{\ell} \varphi\left(\frac{1}{m\|z /\| z\left\|_{2}-x_{i}\right\|_{2}}\right) \mathbb{1}_{\left\{\left(z /\|z\|_{2}\right) \neq x_{i}\right\}}
$$


where $\varphi$ is a continuous decreasing function on $\mathbb{R}^{+}$, such that

$$
\varphi(t):= \begin{cases}1, & \text { if } 0 \leqslant t \leqslant 1 \\ 0, & \text { if } 2 \leqslant t\end{cases}
$$

and $\left(g_{m}\right)_{m \in \mathbb{N}^{*}}$ is a sequence of functions defined on $\mathbb{R}^{2}$ to $[0,1]$ in the following manner

$$
g_{m}(x):=\frac{d\left(x, T^{2 m}\right)}{d\left(x, T^{2 m}\right)+d\left(x, T^{(m)}\right)},
$$

where $d(x, B)$ stands for the distance between the point $x$ and the set $B \subset \mathbb{R}^{2}$. The closed sets $T^{2 m}$ and $T^{(m)}$ are defined by

$$
T^{2 m}:=\left\{x \in \mathbb{R}^{2}, d\left(x, T^{c}\right) \leqslant \frac{1}{2 m}\right\} \text { and } T^{(m)}:=\left\{x \in \mathbb{R}^{2}, d\left(x, T^{c}\right) \geqslant \frac{1}{m}\right\},
$$

$T^{c}$ denoting the complement of $T$ on $\mathbb{R}^{2}$.

We have shown in [10, Lemmas 3.2.1 and 3.2.2] that the functions $\left(g_{m}\right)_{m \in \mathbb{N}^{\star}}$ are well defined, continuous. Thus using the crucial central term in $\varphi$ defining $Y_{m}, m \in \mathbb{N}^{\star}$, one can prove that the r.v. $Y_{m}$ is continuous on $T$. Furthermore we established that the support of function $g_{m}$ restricted to the set $T$ is contained in $T$ for each $m \in \mathbb{N}^{\star}$. This fact implies that $\operatorname{supp}\left(Y_{m}\right) \subset \mathrm{D}_{\mathrm{X}}^{\mathrm{r}}$.

At last this sequence $\left(g_{m}\right)_{m \in \mathbb{N}^{*}}$ is bounded by one and tends to one when $m$ goes to infinity. This implies that the sequence $\left(Y_{m}\right)_{m \in \mathbb{N}^{\star}}$ is bounded on $T$ and that almost surely for all $t \in T, Y_{m}(t) \underset{m \rightarrow+\infty}{\longrightarrow} \breve{Y}(t):=f\left(\nu_{X}(t)\right) \mathbb{1}_{D_{\mathrm{X}}^{\mathrm{r}}}(t) \prod_{i=1}^{\ell} \mathbb{1}_{\left\{\nu_{X}(t) \neq x_{i}\right\}}$.

By summarizing, we have

1. $g:=X$ and $h:=Y_{m}$ verifies assumptions of Theorem 3.3

2. $F_{m}$ and $F-F_{m}$ verify assumptions of Lemma 3.2

3. Almost surely for all $t \in T, Y_{m}(t) \underset{m \rightarrow+\infty}{\longrightarrow} \breve{Y}(t)$

Assertion 1. implies that for all $m \in \mathbb{N}^{\star}$, almost surely

$$
I_{f, m}\left(u_{k}\right) \underset{k \rightarrow+\infty}{\longrightarrow} I_{f, m}(u)
$$

Note that this almost sure convergence is what we need to move forward. Furthermore, assertion 2. implies that for all $m \in \mathbb{N}^{\star}$,

$$
\left\|I_{f, m}\left(u_{k}\right)\right\|_{L^{2}(\Omega)} \underset{k \rightarrow+\infty}{\longrightarrow}\left\|I_{f, m}(u)\right\|_{L^{2}(\Omega)},
$$

so that Scheffé's lemma allows to conclude that for all $m \in \mathbb{N}^{\star}$,

$$
\lim _{k \rightarrow+\infty}\left\|I_{f, m}\left(u_{k}\right)-I_{f, m}(u)\right\|_{L^{2}(\Omega)}=0 .
$$

Now, assertion 2. also implies that for all $m \in \mathbb{N}^{\star}$,

$$
\lim _{k \rightarrow+\infty}\left\|I_{f}\left(u_{k}\right)-I_{f, m}\left(u_{k}\right)\right\|_{L^{2}(\Omega)}=\left\|I_{f}(u)-I_{f, m}(u)\right\|_{L^{2}(\Omega)} .
$$

An upper bound is

$$
\begin{gathered}
\left\|I_{f}\left(u_{k}\right)-I_{f}(u)\right\|_{L^{2}(\Omega)} \leqslant\left\|I_{f}\left(u_{k}\right)-I_{f, m}\left(u_{k}\right)\right\|_{L^{2}(\Omega)}+ \\
\left\|I_{f, m}\left(u_{k}\right)-I_{f, m}(u)\right\|_{L^{2}(\Omega)}+\left\|I_{f, m}(u)-I_{f}(u)\right\|_{L^{2}(\Omega)} .
\end{gathered}
$$


Applying convergence obtained in (3.1) and (3.2), it yields, for all $m \in \mathbb{N}^{\star}$,

$$
\varlimsup_{k \rightarrow+\infty}\left\|I_{f}\left(u_{k}\right)-I_{f}(u)\right\|_{L^{2}(\Omega)} \leqslant 2\left\|I_{f}(u)-I_{f, m}(u)\right\|_{L^{2}(\Omega)} .
$$

Let us set,

$$
I_{f}(u)-I_{f, m}(u)=\int_{\mathcal{C}(u)} \widetilde{Y_{m}}(t) \mathrm{d} \sigma_{1}(t)
$$

where $\widetilde{Y_{m}}(t):=\breve{Y}(t)-Y_{m}(t)$.

By using convergence given in point 3., we obtain that almost surely for all $t \in \mathrm{D}_{\mathrm{X}}^{\mathrm{r}}$, $\lim _{m \rightarrow+\infty} \tilde{Y}_{m}(t)=0$.

Furthermore for all $t \in \mathrm{D}_{\mathrm{X}}^{\mathrm{r}}$, one has $\left|\widetilde{Y}_{m}(t)\right| \leqslant$ C. Moreover by applying Remark 2.9, one has

$$
\mathbb{E}\left[\sigma_{1}(\mathrm{C}(u))\right]^{2}<+\infty
$$

and then almost surely $\sigma_{1}(\mathrm{C}(u))<+\infty$. The Lebesgue convergence theorem induces that

- Almost surely, $\lim _{m \rightarrow+\infty}\left(I_{f}(u)-I_{f, m}(u)\right)=0$. Furthermore

- Almost surely, $\left|I_{f}(u)-I_{f, m}(u)\right| \leqslant \mathbf{C} \sigma_{1}(\mathrm{C}(u)) \in L^{2}(\mathrm{dP})$, last belonging providing from (3.4).

Applying once again the Lebesgue convergence theorem, one has finally proved that $\lim _{m \rightarrow+\infty}\left\|I_{f, m}(u)-I_{f}(u)\right\|_{L^{2}(\Omega)}=0$. Thus by using inequality (3.3), one concludes that

$$
\varlimsup_{k \rightarrow+\infty}\left\|I_{f}\left(u_{k}\right)-I_{f}(u)\right\|_{L^{2}(\Omega)}=0
$$

yielding Theorem 3.1.

We are now ready to define a $L^{2}(\Omega)$-expansion for $J_{f}(u)$.

\subsection{Hermite expansion for general functionals}

Let $f: S^{1} \rightarrow \mathbb{R}$ be in $\mathcal{B}_{1}$ and $u$ a fixed level in $\mathbb{R}$. We define an approximation of $J_{f}(u)$. For $\sigma>0$, we define

$$
J_{f}(u, \sigma):=\frac{1}{\sigma} \int_{-\infty}^{+\infty} K\left(\frac{u-v}{\sigma}\right) J_{f}(v) \mathrm{d} v
$$

where $K$ is a continuous density function with a compact support in $[-1,1]$. In view of applying the coarea formula to $J_{f}(u, \sigma)$ we recall a statement of this formula, adapted to our situation and that can be found in Federer [15, Theorem 3.2.12] and also in Berzin et al. [10, Corollary 2.1.1].

Corollary 3.4. (Coarea formula) Let $h$ a measurable function, $h: \mathbb{R}^{2} \times \mathbb{R} \rightarrow \mathbb{R}$ and $g: T \subset \mathbb{R}^{2} \rightarrow \mathbb{R}$ be a function belonging to $\boldsymbol{C}^{1}(T)$. We have

$$
\int_{T} h(t, g(t))\|\nabla g(t)\|_{2} \mathrm{~d} t=\int_{-\infty}^{+\infty}\left[\int_{\mathcal{C}_{T, g}^{D^{r}}(y)} h(t, y) \mathrm{d} \sigma_{1}(t)\right] \mathrm{d} y,
$$

provided that one of the two integrals is finite.

By applying last corollary to the measurable function $h: \mathbb{R}^{2} \times \mathbb{R} \rightarrow \mathbb{R}$ defined as:

$$
h(t, y):=\frac{1}{\sigma_{2}(T)} f\left(\nu_{X}(t)\right) \mathbb{1}_{\{\nabla X(t) \neq 0\}} \frac{1}{\sigma} K\left(\frac{u-y}{\sigma}\right),
$$


to the $\mathbf{C}^{1}$-function $g:=X: T \subset \mathbb{R}^{2} \rightarrow \mathbb{R}$, we get:

$$
J_{f}(u, \sigma)=\frac{1}{\sigma_{2}(T)} \int_{T} f\left(\nu_{X}(t)\right) \mathbb{1}_{\{\nabla X(t) \neq 0\}} \frac{1}{\sigma} K\left(\frac{u-X(t)}{\sigma}\right)\|\nabla X(t)\|_{2} \mathrm{~d} t<+\infty .
$$

Let $\Lambda:=\left(\begin{array}{cc}\lambda_{1} & 0 \\ 0 & \lambda_{2}\end{array}\right)$, a matrix that factorizes $A$ such that $A=P \Lambda P^{t}$, where $P$ is a unitary matrix and ${ }^{t}$ stands for the transpose symbol.

The matrix $\Gamma^{\boldsymbol{X}}$ stands for the covariance matrix of the 3-dimensional Gaussian vector

$$
\boldsymbol{X}(t):=(\nabla X(t), X(t))^{t} .
$$

We recall that since $Z$ is isotropic, $\mathbb{E}\left[\nabla Z(0) \nabla Z(0)^{t}\right]=\mu I_{2}$, where $\mu:=-\frac{\partial^{2} r_{z}}{\partial t_{i}^{2}}(0)$ for any $i=1,2$ and $I_{2}$ stands for the identity matrix in $\mathbb{R}^{2}$.

We denote $\Delta$ for the $3 \times 3$ matrix, $\Delta:=\left(\begin{array}{cc}\sqrt{\mu} P \Lambda & 0 \\ 0 & \sqrt{r_{z}(0)}\end{array}\right)$. It is such that $\Delta \Delta^{t}=\Gamma^{\boldsymbol{X}}$. We have seen in Section 2.2 (see Remark 2.11) that $Z$ admits a spectral density $f_{z}$ and then the same fact occurs for $X$. We have noted $f_{x}$ the spectral density for $X$.

We obtain the following spectral representations: for $t \in \mathbb{R}^{2}$,

$$
X(t)=\int_{\mathbb{R}^{2}} e^{i\langle t, \lambda\rangle} \sqrt{f_{x}(\lambda)} \mathrm{d} W(\lambda)
$$

where $W$ stands for the standard Brownian motion.

Thus, for any $\lambda:=\left(\lambda_{i}\right)_{1 \leqslant i \leqslant 2}$ in $\mathbb{R}^{2}$, we let

$$
\nu(\lambda):=\left(i \lambda_{1}, i \lambda_{2}, 1\right)^{t}
$$

so that for any $t \in \mathbb{R}^{2}$,

$$
U(t)=\left(U_{i}(t)\right)_{1 \leqslant i \leqslant 3}:=\Delta^{-1} \cdot \boldsymbol{X}(t)=\int_{\mathbb{R}^{2}} e^{i\langle t, \lambda\rangle} \sqrt{f_{x}(\lambda)} \Delta^{-1} \cdot \nu(\lambda) \mathrm{d} W(\lambda) .
$$

We can write for any $t \in \mathbb{R}^{2}, \boldsymbol{X}(t)=\Delta . U(t)$, where $U(t)$ is a 3 -dimensional standard Gaussian vector.

With these notations, one obtains:

$$
\begin{aligned}
J_{f}(u, \sigma)=\frac{\sqrt{\mu}}{\sigma_{2}(T)} \frac{1}{\sigma} \int_{T} f\left(\frac{P \Lambda \cdot\left(\begin{array}{c}
U_{1}(t) \\
U_{2}(t)
\end{array}\right)}{\sqrt{\lambda_{1}^{2} U_{1}^{2}(t)+\lambda_{2}^{2} U_{2}^{2}(t)}}\right) & \mathbb{1}_{\left\{U_{1}(t) \neq 0 \text { or } U_{2}(t) \neq 0\right\}} \\
& \times K\left(\frac{u-\sqrt{r_{z}(0)} U_{3}(t)}{\sigma}\right) \sqrt{\lambda_{1}^{2} U_{1}^{2}(t)+\lambda_{2}^{2} U_{2}^{2}(t)} \mathrm{d} t .
\end{aligned}
$$

We define for $\sigma>0$ and $y=\left(y_{i}\right)_{1 \leqslant i \leqslant 3} \in \mathbb{R}^{3}$, the map $g_{\sigma}$ as:

$$
g_{\sigma}(y):=\frac{\sqrt{\mu}}{\sigma} K\left(\frac{u-\sqrt{r_{z}(0)} y_{3}}{\sigma}\right) f\left(\frac{P \Lambda \cdot\left(\begin{array}{l}
y_{1} \\
y_{2}
\end{array}\right)}{\sqrt{\lambda_{1}^{2} y_{1}^{2}+\lambda_{2}^{2} y_{2}^{2}}}\right) \sqrt{\lambda_{1}^{2} y_{1}^{2}+\lambda_{2}^{2} y_{2}^{2}} \mathbb{1}_{\left\{y_{1} \neq 0 \text { or } y_{2} \neq 0\right\}} .
$$

Since the map belongs to $L^{2}\left(\mathbb{R}^{3}, \phi_{3}(y) \mathrm{d} y\right)$, the following expansion converges in this space:

$$
g_{\sigma}(y)=\sum_{q=0}^{+\infty} \sum_{\substack{\boldsymbol{k} \in \mathbb{N}^{3} \\|\boldsymbol{k}|=q}} a_{f, \sigma}(\boldsymbol{k}, u) \widetilde{H}_{\boldsymbol{k}}(y)
$$


while taking $\boldsymbol{k}:=\left(k_{i}\right)_{1 \leqslant i \leqslant 3} \in \mathbb{N}^{3}$,

$$
a_{f, \sigma}(\boldsymbol{k}, u):=a_{f}\left(k_{1}, k_{2}\right) a_{\sigma}\left(k_{3}, u\right),
$$

where for $\left(k_{1}, k_{2}\right) \in \mathbb{N}^{2}$,

$$
\begin{array}{r}
a_{f}\left(k_{1}, k_{2}\right):=\frac{\sqrt{\mu}}{k_{1} ! k_{2} !} \int_{\mathbb{R}^{2}} f\left(\frac{P \Lambda \cdot\left(\begin{array}{l}
y_{1} \\
y_{2}
\end{array}\right)}{\sqrt{\lambda_{1}^{2} y_{1}^{2}+\lambda_{2}^{2} y_{2}^{2}}}\right) \sqrt{\lambda_{1}^{2} y_{1}^{2}+\lambda_{2}^{2} y_{2}^{2}} H_{k_{1}}\left(y_{1}\right) \phi\left(y_{1}\right) \\
H_{k_{2}}\left(y_{2}\right) \phi\left(y_{2}\right) \mathrm{d} y_{1} \mathrm{~d} y_{2}
\end{array}
$$

and

$$
a_{\sigma}\left(k_{3}, u\right):=\frac{1}{k_{3} !} \int_{-\infty}^{+\infty} \frac{1}{\sigma} K\left(\frac{u-\sqrt{r_{z}(0)} y}{\sigma}\right) H_{k_{3}}(y) \phi(y) \mathrm{d} y
$$

In this way, we obtain the expansion of the functional $J_{f}(u, \sigma)$ in $L^{2}(\Omega)$, that is:

$$
J_{f}(u, \sigma) \stackrel{L^{2}(\Omega)}{=} \frac{1}{\sigma_{2}(T)} \sum_{q=0}^{+\infty} \sum_{\substack{\boldsymbol{k} \in \mathbb{N}^{3} \\|\boldsymbol{k}|=q}} a_{f, \sigma}(\boldsymbol{k}, u) \int_{T} \widetilde{H}_{\boldsymbol{k}}(U(t)) \mathrm{d} t .
$$

Now observe that as $\sigma$ tends to zero, $a_{\sigma}\left(k_{3}, u\right) \rightarrow a\left(k_{3}, u\right)$, where coefficient $a\left(k_{3}, u\right)$ is defined as

$$
a\left(k_{3}, u\right):=\frac{1}{k_{3} !} H_{k_{3}}\left(\frac{u}{\sqrt{r_{z}(0)}}\right) \phi\left(\frac{u}{\sqrt{r_{z}(0)}}\right) \frac{1}{\sqrt{r_{z}(0)}} .
$$

This remark will yield via the forthcoming theorem the following expansion in $L^{2}(\Omega)$ of $J_{f}(u)$.

Theorem 3.5. For $f: S^{1} \rightarrow \mathbb{R}$ in $\mathcal{B}_{1}$ and $u$ a fixed level in $\mathbb{R}$, one has the following expansion in $L^{2}(\Omega)$,

$$
J_{f}(u)=\frac{1}{\sigma_{2}(T)} \sum_{q=0}^{+\infty} \sum_{\substack{\boldsymbol{k} \in \mathbb{N}^{3} \\|\boldsymbol{k}|=q}} a_{f}(\boldsymbol{k}, u) \int_{T} \widetilde{H}_{\boldsymbol{k}}(U(t)) \mathrm{d} t,
$$

where coefficients $a_{f}(\boldsymbol{k}, u)$ are defined by

$$
a_{f}(\boldsymbol{k}, u):=a_{f}\left(k_{1}, k_{2}\right) a\left(k_{3}, u\right),
$$

with $a_{f}\left(k_{1}, k_{2}\right)$ and $a\left(k_{3}, u\right)$ previously respectively defined by (3.6) and (3.8).

Remark 3.6. This equality has nothing obvious because we do not know a priori that the right member in the last equality really belongs to $L^{2}(\Omega)$. This fact comes from the way we obtained this expansion, the series $\sum_{k_{3}=0}^{+\infty} a^{2}\left(k_{3}, u\right) k_{3}$ ! being equal to $+\infty$ as the Hermite development in $L^{2}(\mathbb{R}, \phi(x) \mathrm{d} x)$ of delta's Dirac function in point $u$.

Proof of Theorem 3.5. The proof of this theorem strongly leans on that given in Estrade and León [14] and extensively uses the orthogonality of the various chaos.

We demonstrate Proposition 3.7. 
Proposition 3.7. For $f: S^{1} \rightarrow \mathbb{R}$ in $\mathcal{B}_{1}$ and $u$ a fixed level in $\mathbb{R}$, one has the following convergence,

$$
J_{f}(u, \sigma) \underset{\sigma \rightarrow 0}{\stackrel{L^{2}(\Omega)}{\longrightarrow}} J_{f}(u)
$$

Proof of Proposition 3.7. One can easily see that the proof of this proposition follows immediately from that of Theorem 3.1.

As in the proof of [14, Proposition 1.3], let us define formally

$$
\eta(T):=\frac{1}{\sigma_{2}(T)} \sum_{q=0}^{+\infty} \sum_{\substack{\boldsymbol{k} \in \mathbb{N}^{3} \\|\boldsymbol{k}|=q}} a_{f}(\boldsymbol{k}, u) \int_{T} \widetilde{H}_{\boldsymbol{k}}(U(t)) \mathrm{d} t
$$

and prove the following lemma.

Lemma 3.8. $\eta(T) \in L^{2}(\Omega)$.

Proof of Lemma 3.8. First, remark that if $\boldsymbol{k}, \boldsymbol{m} \in \mathbb{N}^{3}$ are such that $|\boldsymbol{k}| \neq|\boldsymbol{m}|$, then for all $s, t \in T \times T, \mathbb{E}\left[\widetilde{H}_{\boldsymbol{k}}(U(t)) \widetilde{H}_{\boldsymbol{m}}(U(s))\right]=0$ and the above expression, $\eta(T)$ turns out to be a sum of orthogonal terms in $L^{2}(\Omega)$.

Indeed, to prove this we need a generalization of Mehler's formula given in Azaïs and Wschebor [8, Lemma 10.7, part (b), page 269] via the following lemma proved in Appendix A.

Lemma 3.9. Let $X=\left(X_{i}\right)_{i=1,2,3}$ and $Y=\left(Y_{j}\right)_{j=1,2,3}$ be two centred standard Gaussian vectors in $\mathbb{R}^{3}$ such that for $1 \leqslant i, j \leqslant 3, \mathbb{E}\left[X_{i} Y_{j}\right]=\rho_{i j}$, then for $\boldsymbol{k}, \boldsymbol{m} \in \mathbb{N}^{3}$, one has

$$
\mathbb{E}\left[\widetilde{H}_{\boldsymbol{k}}(X) \widetilde{H}_{\boldsymbol{m}}(Y)\right]=\left(\sum_{\substack{d_{i j} \geqslant 0 \\ \sum_{j} d_{i j}=k_{i} \\ \sum_{i} d_{i j}=m_{j}}} \boldsymbol{k} ! \boldsymbol{m} ! \prod_{1 \leqslant i, j \leqslant 3} \frac{\rho_{i j}^{d_{i j}}}{d_{i j} !}\right) \mathbb{1}_{|\boldsymbol{k}|=|\boldsymbol{m}|}
$$

As in [14], let us fix $Q \in \mathbb{N}$ and let us denote by $\pi^{Q}$ the projection onto the first $Q$ chaos in $L^{2}(\Omega)$ and by $\pi_{Q}$ the projection onto the remaining one. With these notations one has

$$
\pi^{Q}(\eta(T))=\frac{1}{\sigma_{2}(T)} \sum_{q=0}^{Q} \sum_{\substack{\boldsymbol{k} \in \mathbb{N}^{3} \\|\boldsymbol{k}|=q}} a_{f}(\boldsymbol{k}, u) \int_{T} \widetilde{H}_{\boldsymbol{k}}(U(t)) \mathrm{d} t
$$

and $\pi_{Q}(\eta(T))=\eta(T)-\pi^{Q}(\eta(T))$.

By the precedent remark, we have

$$
\mathbb{E}\left[\pi^{Q}(\eta(T))\right]^{2}=\sum_{q=0}^{Q} \mathbb{E}\left[\frac{1}{\sigma_{2}(T)} \sum_{\substack{\boldsymbol{k} \in \mathbb{N}^{3} \\|\boldsymbol{k}|=q}} a_{f}(\boldsymbol{k}, u) \int_{T} \widetilde{H}_{\boldsymbol{k}}(U(t)) \mathrm{d} t\right]^{2} .
$$

Remember that coefficients $a_{f, \sigma}(\boldsymbol{k}, u)$ have been defined by (3.5). 
Applying the Fatou's lemma, since $\lim _{\sigma \rightarrow 0} a_{f, \sigma}(\boldsymbol{k}, u)=a_{f}(\boldsymbol{k}, u)$, we obtain

$$
\begin{aligned}
\mathbb{E}\left[\pi^{Q}(\eta(T))\right]^{2} & \leqslant \underline{\lim _{\sigma \rightarrow 0}} \sum_{q=0}^{Q} \mathbb{E}\left[\frac{1}{\sigma_{2}(T)} \sum_{\substack{\boldsymbol{k} \in \mathbb{N}^{3} \\
|\boldsymbol{k}|=q}} a_{f, \sigma}(\boldsymbol{k}, u) \int_{T} \widetilde{H}_{\boldsymbol{k}}(U(t)) \mathrm{d} t\right]^{2} \\
& \leqslant \underline{\lim _{\sigma \rightarrow 0}} \sum_{q=0}^{+\infty} \mathbb{E}\left[\frac{1}{\sigma_{2}(T)} \sum_{\substack{\boldsymbol{k} \in \mathbb{N}^{3} \\
|\boldsymbol{k}|=q}} a_{f, \sigma}(\boldsymbol{k}, u) \int_{T} \widetilde{H}_{\boldsymbol{k}}(U(t)) \mathrm{d} t\right]^{2} \\
& =\varliminf_{\sigma \rightarrow 0} \mathbb{E}\left[\frac{1}{\sigma_{2}(T)} \sum_{q=0}^{+\infty} \sum_{\substack{\boldsymbol{k} \in \mathbb{N}^{3} \\
|\boldsymbol{k}|=q}} a_{f, \sigma}(\boldsymbol{k}, u) \int_{T} \widetilde{H}_{\boldsymbol{k}}(U(t)) \mathrm{d} t\right]^{2} \\
& =\underline{\lim _{\sigma \rightarrow 0}} \mathbb{E}\left[J_{f}(u, \sigma)\right]^{2}=\mathbb{E}\left[J_{f}(u)\right]^{2}<+\infty
\end{aligned}
$$

the two last equalities providing from equality (3.7) and from Proposition 3.7 and the third last equality from the expansion in $L^{2}(\Omega)$ of $J_{f}(u, \sigma)$ (see (3.7)) and precedent remark. The finiteness of $\mathbb{E}\left[J_{f}(u)\right]^{2}$ comes from Lemma 3.2.

Thus the random variable $\eta(T) \in L^{2}(\Omega)$ and

$$
\mathbb{E}[\eta(T)]^{2}=\sum_{q=0}^{+\infty} \mathbb{E}\left[\frac{1}{\sigma_{2}(T)} \sum_{\substack{\boldsymbol{k} \in \mathbb{N}^{3} \\|\boldsymbol{k}|=q}} a_{f}(\boldsymbol{k}, u) \int_{T} \widetilde{H}_{\boldsymbol{k}}(U(t)) \mathrm{d} t\right]^{2}
$$

This achieves proof of Lemma 3.8.

It remains to prove that $J_{f}(u)=\eta(T)$ in $L^{2}(\Omega)$.

As in the proof of Theorem 3.1, we write $\|\cdot\|_{L^{2}(\Omega)}$ for the $L^{2}(\Omega)$-norm.

For fixed $Q \in \mathbb{N}$ and $\sigma>0$, one has the following inequalities

$$
\begin{aligned}
&\left\|J_{f}(u)-\eta(T)\right\|_{L^{2}(\Omega)} \leqslant\left\|\pi_{Q}\left(J_{f}(u)-\eta(T)\right)\right\|_{L^{2}(\Omega)} \\
&+\left\|\pi^{Q}\left(J_{f}(u)-J_{f}(u, \sigma)\right)\right\|_{L^{2}(\Omega)}+\left\|\pi^{Q}\left(J_{f}(u, \sigma)-\eta(T)\right)\right\|_{L^{2}(\Omega)} \\
& \leqslant\left\|\pi_{Q}\left(J_{f}(u)\right)\right\|_{L^{2}(\Omega)}+\left\|\pi_{Q}(\eta(T))\right\|_{L^{2}(\Omega)}+\left\|J_{f}(u)-J_{f}(u, \sigma)\right\|_{L^{2}(\Omega)} \\
&+\left\|\pi^{Q}\left(J_{f}(u, \sigma)-\eta(T)\right)\right\|_{L^{2}(\Omega)}
\end{aligned}
$$

Now by Lemma 3.2 and Lemma 3.8, $J_{f}(u)$ and $\eta(T)$ belong to $L^{2}(\Omega)$, thus $\lim _{Q \rightarrow+\infty}\left\|\pi_{Q}\left(J_{f}(u)\right)\right\|_{L^{2}(\Omega)}=\lim _{Q \rightarrow+\infty}\left\|\pi_{Q}(\eta(T))\right\|_{L^{2}(\Omega)}=0$.

Furthermore due to Proposition 3.7, $\lim _{\sigma \rightarrow 0}\left\|J_{f}(u)-J_{f}(u, \sigma)\right\|_{L^{2}(\Omega)}=0$ and for fixed $Q \in \mathbb{N}$ and since $\lim _{\sigma \rightarrow 0} a_{f, \sigma}(\boldsymbol{k}, u)=a_{f}(\boldsymbol{k}, u), \lim _{\sigma \rightarrow 0}\left\|\pi^{Q}\left(J_{f}(u, \sigma)-\eta(T)\right)\right\|_{L^{2}(\Omega)}=0$.

Hence, for fixed $Q \in \mathbb{N}$ and taking limit as $\sigma$ tends to zero one obtains,

$$
\left\|J_{f}(u)-\eta(T)\right\|_{L^{2}(\Omega)} \leqslant\left\|\pi_{Q}\left(J_{f}(u)\right)\right\|_{L^{2}(\Omega)}+\left\|\pi_{Q}(\eta(T))\right\|_{L^{2}(\Omega)} .
$$

Then, taking limit as $Q$ tends to infinity one finally gets

$$
\left\|J_{f}(u)-\eta(T)\right\|_{L^{2}(\Omega)}=0
$$

Theorem 3.5 ensues. 


\subsection{One order Rice formula}

As by product of Theorem 3.1, we obtain the one order Rice formula for the general functional $J_{f}(u), u$ being a fixed level in $\mathbb{R}$. More precisely we have the following proposition.

Proposition 3.10. For $f: S^{1} \rightarrow \mathbb{R}$ in $\mathcal{B}_{1}$ and $u$ a fixed level in $\mathbb{R}$, one has

$$
\mathbb{E}\left[J_{f}(u)\right]=p_{X(0)}(u) \mathbb{E}\left[f\left(\frac{\nabla X(0)}{\|\nabla X(0)\|_{2}}\right)\|\nabla X(0)\|_{2}\right],
$$

where $p_{X(0)}(\cdot)$ stands for the density of $X(0)$.

Proof of Proposition 3.10. First let us suppose that $f: S^{1} \rightarrow \mathbb{R}$ is a continuous and bounded function. We refer to Remark 2.6 following Theorem 2.5 stated in Section 2.1 and we check that processes $X$ and $\widetilde{Y}:=f\left(\nu_{X}\right) \mathbb{1}_{\mathrm{D}_{\mathrm{X}}^{\mathrm{r}}}$ verify assumptions given in this theorem, that is assumptions $\mathbf{H}_{1}, \mathbf{H}_{2}, \mathbf{H}_{\mathbf{4}}$ and $\mathbf{H}_{5}$.

Theorem 3.1 implies that assumptions $\mathbf{H}_{\mathbf{2}}$ and $\mathbf{H}_{\mathbf{4}}$ are satisfied, assumption $\mathbf{H}_{\mathbf{1}}$ being fulfilled.

Thus we just check if the hypothesis $\mathbf{H}_{\mathbf{5}}$ is verified. In this context, let us compute the following integral:

$$
\begin{array}{r}
\int_{T} p_{X(t)}(u) \mathbb{E}\left[\tilde{Y}(t)\|\nabla X(t)\|_{2} \mid X(t)=u\right] \mathrm{d} t= \\
\sigma_{2}(T) p_{X(0)}(u) \mathbb{E}\left[f\left(\frac{\nabla X(0)}{\|\nabla X(0)\|_{2}}\right)\|\nabla X(0)\|_{2}\right] .
\end{array}
$$

Since function $u \longmapsto p_{X(0)}(u)$ is continuous, hypothesis $\mathbf{H}_{\mathbf{5}}$ is satisfied. All conditions are met to apply the Rice formula, and one gets,

$$
\begin{aligned}
\mathbb{E}\left[J_{f}(u)\right] & =\frac{1}{\sigma_{2}(T)} \int_{T} p_{X(t)}(u) \mathbb{E}\left[f\left(\frac{\nabla X(t)}{\|\nabla X(t)\|_{2}}\right) \mathbb{1}_{\mathrm{D}_{\mathrm{X}}^{\mathrm{r}}}(t)\|\nabla X(t)\|_{2} \mid X(t)=u\right] \mathrm{d} t \\
& =p_{X(0)}(u) \mathbb{E}\left[f\left(\frac{\nabla X(0)}{\|\nabla X(0)\|_{2}}\right)\|\nabla X(0)\|_{2}\right] .
\end{aligned}
$$

Now let us suppose that function $f$ belongs to $\mathcal{B}_{1}$. Let us note $\left\{x_{1}, \cdots, x_{\ell}\right\}, \ell \in \mathbb{N}^{\star}$, the finite set of the points of the sphere $S^{1}$, where $f$ is discontinuous. By Remark 2.13, almost surely $J_{f}(u)=\int_{\mathcal{C}(u)} f\left(\nu_{X}(t)\right) \prod_{i=1}^{\ell} \mathbb{1}_{\left\{\nu_{X}(t) \neq x_{i}\right\}} \mathrm{d} \sigma_{1}(t)$.

As in the proof of Theorem 3.1 (and Lemma 3.2), let us consider the following approximation.

For $m \in \mathbb{N}^{\star}$, let

$$
J_{m}(u):=\frac{1}{\sigma_{2}(T)} \int_{\mathcal{C}(u)} f_{m}\left(\nu_{X}(t)\right) \mathrm{d} \sigma_{1}(t),
$$

where we define for $w \in S^{1}$,

$$
f_{m}(w):=f(w) \prod_{i=1}^{\ell} \varphi\left(\frac{1}{m\left\|w-x_{i}\right\|_{2}}\right) \mathbb{1}_{\left\{w \neq x_{i}\right\}},
$$

and $\varphi$ has been defined in the proof of Theorem 3.1. Since $f_{m}: S^{1} \rightarrow \mathbb{R}$ is a bounded and continuous function, the first part of this proof entails that

$$
\mathbb{E}\left[J_{m}(u)\right]=p_{X(0)}(u) \mathbb{E}\left[f_{m}\left(\frac{\nabla X(0)}{\|\nabla X(0)\|_{2}}\right)\|\nabla X(0)\|_{2}\right]
$$

On the one hand, we observe that for all $w \in S^{1}, f_{m}(w) \underset{m \rightarrow+\infty}{\longrightarrow} f(w) \prod_{i=1}^{\ell} \mathbb{1}_{\left\{w \neq x_{i}\right\}}$. 
On the other hand, by using that function $f_{m}$ is bounded and that $\mathbb{E}\left[\sigma_{1}(\mathrm{C}(u))\right]<+\infty$, we deduce by applying the Lebesgue convergence theorem that

$$
\begin{aligned}
\mathbb{E}\left[J_{f}(u)\right] & =p_{X(0)}(u) \mathbb{E}\left[f\left(\frac{\nabla X(0)}{\|\nabla X(0)\|_{2}}\right) \prod_{i=1}^{\ell} \mathbb{1}_{\left\{\frac{\nabla X(0)}{\|\nabla X(0)\|_{2}} \neq x_{i}\right\}}\|\nabla X(0)\|_{2}\right] \\
& =p_{X(0)}(u) \mathbb{E}\left[f\left(\frac{\nabla X(0)}{\|\nabla X(0)\|_{2}}\right)\|\nabla X(0)\|_{2}\right] .
\end{aligned}
$$

The proof is completed.

\section{Convergence of general level functionals}

In this section $\left(T_{n}\right)_{n}$ will be open bounded squares of $\mathbb{R}^{2}$, with the following form $\left.T_{n}:=\right]-n, n\left[^{2}\right.$ with $n \in \mathbb{N}^{\star}$, and $n$ tends to infinity. Also let $u$ be a fixed level in $\mathbb{R}$.

Remember that for a function $f, f: S^{1} \rightarrow \mathbb{R}, f \in \mathcal{B}_{1}$, we have defined in Section 2.1 the following general functional $J_{f}^{(n)}(u)$ of the level $u$ as:

$$
J_{f}^{(n)}(u):=\frac{1}{(2 n)^{2}} \int_{\mathcal{C}_{n}(u)} f\left(\nu_{X}(t)\right) \mathrm{d} \sigma_{1}(t)
$$

where $\nu_{X}(t)=\frac{\nabla X(t)}{\|\nabla X(t)\|_{2}}$.

Our objective consists now to establish the almost sure convergence of such functionals and also their asymptotic normality, when the observation window $T_{n}$ tends to $\mathbb{R}^{2}$ as $n$ tends to infinity. To this end we will use the results established in previous Section 3, that is the Hermite expansion of the random variables $J_{f}^{(n)}(u)$ and also the one order Rice formula in order to compute their expectation.

\subsection{Almost sure convergence for $J_{f}^{(n)}(u)$}

By applying an ergodic theorem for stationary processes (Adler [1, §6.5)]), we shall show the following general almost sure convergence theorem.

Theorem 4.1. For $f: S^{1} \rightarrow \mathbb{R}$ a function belonging to $\mathcal{B}_{1}$,

$$
J_{f}^{(n)}(u) \underset{n \rightarrow+\infty}{\stackrel{a . s .}{\longrightarrow}} \mathbb{E}\left[J_{f}^{(1)}(u)\right] .
$$

Proof of Theorem 4.1. Let $f: S^{1} \rightarrow \mathbb{R}$ a function that belongs to $\mathcal{B}_{1}$. As a first step we suppose that function $f$ is non-negative and that the square $T_{n}$ has the following shape: $\left.T_{n}:=\right] 0, n[\times] 0, n[$. Lemma 4.2 is proved in Appendix A.

Lemma 4.2. Let $f: S^{1} \rightarrow \mathbb{R}$ be a non-negative function belonging to $\mathcal{B}_{1}$. One has

$$
\begin{gathered}
\int_{0}^{n-1} \int_{0}^{n-1} \int_{\mathcal{C}_{[t, t+1[\times[s, s+1[}(u)} f\left(\nu_{X}(x)\right) \mathrm{d} \sigma_{1}(x) \mathrm{d} t \mathrm{~d} s \\
\quad \leqslant \int_{\mathcal{C}_{T_{n}}(u)} f\left(\nu_{X}(x)\right) \mathrm{d} \sigma_{1}(x) \leqslant \\
\int_{0}^{n+1} \int_{0}^{n+1} \int_{C_{[t-1, t[\times[s-1, s[}(u)}^{1} f\left(\nu_{X}(x)\right) \mathrm{d} \sigma_{1}(x) \mathrm{d} t \mathrm{~d} s
\end{gathered}
$$

Let us note $H(t, s):=\int_{\mathcal{C}_{] 0, t[\times] 0, s[}(u)} f\left(\nu_{X}(x)\right) \mathrm{d} \sigma_{1}(x)$. 
On the one hand, since function $f$ is bounded, we have the convergence that follows

$$
\frac{1}{(2 n)^{2}} \int_{0}^{1} \int_{0}^{1} H(t, s) \mathrm{d} t \mathrm{~d} s \leqslant \frac{\mathbf{C}}{(2 n)^{2}} \sigma_{1}\left(\mathcal{C}_{] 0,1\left[\left[^{2}\right.\right.}(u)\right) \underset{n \rightarrow+\infty}{\stackrel{a . s .}{\longrightarrow}} 0
$$

last convergence providing from Remark 2.9. Indeed, since $\mathbb{E}\left[\sigma_{1}\left(\mathcal{C}_{0,1\left[^{2}\right.}(u)\right)\right]^{2}<+\infty$, we deduce that $\sigma_{1}\left(\mathcal{C}_{0,1\left[^{2}\right.}(u)\right)$ is almost surely finite. On the other hand, noting by

$$
\xi(t, s):=\int_{\mathcal{C}_{[t, t+1[\times[s, s+1[}(u)} f\left(\nu_{X}(x)\right) \mathrm{d} \sigma_{1}(x),
$$

one has

$$
\begin{aligned}
\frac{1}{(2 n)^{2}} \int_{0}^{n-1} \int_{0}^{n-1} \int_{\mathcal{C}_{[t, t+1[\times[s, s+1[}(u)} f\left(\nu_{X}(x)\right) \mathrm{d} \sigma_{1}(x) \mathrm{d} t \mathrm{~d} s= \\
\left(\frac{n-1}{2 n}\right)^{2} \frac{1}{(n-1)^{2}} \int_{0}^{n-1} \int_{0}^{n-1} \xi(t, s) \mathrm{d} t \mathrm{~d} s .
\end{aligned}
$$

Since process $X$ is a centred stationary Gaussian process with continuous trajectories such that $r_{x}(t)$ tends to zero as $\|t\|_{2}$ tends to $+\infty$, we deduce from [1, Theorem 6.5.4] that process $X$ is ergodic. Now since process $X$ is strictly stationary and ergodic, we deduce from [1, Theorem 6.5.2], that process $\xi(t, s)$ is a strictly stationary ergodic process. Note that the set $\left[t, t+1\left[\times\left[s, s+1\left[\right.\right.\right.\right.$ is not an open set of $\mathbb{R}^{2}$. However Proposition 3.10 still remains valid. That is the closure of this rectangle does not play role in the expression of the one order Rice formula. Thus from Proposition 3.10 we know that $\mathbb{E}[|\xi(t, s)|]<+\infty$. By [1, Theorem 6.5.1], we deduce that

$$
\left(\frac{n-1}{2 n}\right)^{2} \frac{1}{(n-1)^{2}} \int_{0}^{n-1} \int_{0}^{n-1} \xi(t, s) \mathrm{d} t \mathrm{~d} s \underset{n \rightarrow+\infty}{\stackrel{a . s .}{\longrightarrow}} \frac{1}{4} \mathbb{E}[\xi(0,0)]=\frac{1}{4} \mathbb{E}\left[J_{f}^{(1)}(u)\right] .
$$

In the same way, one obtains

$$
\begin{aligned}
&\left(\frac{n+1}{2 n}\right)^{2} \frac{1}{(n+1)^{2}} \int_{0}^{n+1} \int_{0}^{n+1} \int_{\mathrm{C}_{[t-1, t[\times[s-1, s[}(u)} f\left(\nu_{X}(x)\right) \mathrm{d} \sigma_{1}(x) \mathrm{d} t \mathrm{~d} s \\
& \stackrel{\text { a.s. }}{n \rightarrow+\infty} \frac{1}{4} \mathbb{E}\left[J_{f}^{(1)}(u)\right] .
\end{aligned}
$$

Finally, by using Lemma 4.2 , one proved that

$$
\frac{1}{(2 n)^{2}} \int_{\mathcal{C}_{] 0, n\left[^{2}\right.}(u)} f\left(\nu_{X}(x)\right) \mathrm{d} \sigma_{1}(x) \underset{n \rightarrow+\infty}{\stackrel{a . s .}{\longrightarrow}} \frac{1}{4} \mathbb{E}\left[J_{f}^{(1)}(u)\right]
$$

Now, working in a similar way succesively with $\left.\left.\left.T_{n}:=\right]-n, 0\right] \times\right] 0, n\left[\right.$ or $\left.\left.\left.\left.T_{n}:=\right]-n, 0\right] \times\right]-n, 0\right]$ or still with $\left.\left.T_{n}=\right] 0, n[\times]-n, 0\right]$, one should prove the same convergence result for each square $T_{n}$, even if $T_{n}$ is not an open set of $\mathbb{R}^{2}$. Finally, if $f$ is a non-negative function, by using the linearity of the interest functional one have proved that

$$
J_{f}^{(n)}(u) \underset{n \rightarrow+\infty}{\stackrel{a . s .}{\longrightarrow}} 4 \times \frac{1}{4} \mathbb{E}\left[J_{f}^{(1)}(u)\right]=\mathbb{E}\left[J_{f}^{(1)}(u)\right] .
$$

To conclude the proof of Theorem 4.1, we decompose function $f$ into its negative part and into its positive part, that is under the shape, $f=f^{+}-f^{-}$, and we apply the previous result to each of the interest functionals $J_{f^{+}}^{(n)}(u)$ and $J_{f^{-}}^{(n)}(u)$. 


\subsection{Convergence in law for $\xi_{f}^{(n)}(u)$}

We establish a CLT for a centred and suitably rescalled general functional $J_{f}^{(n)}(u)$, function $f: S^{1} \rightarrow \mathbb{R}$ being any function in $\mathcal{B}_{1}$ and $u$ a fixed level in $\mathbb{R}$. Roughly speaking we decided to give the rate of convergence in Theorem 4.1. In this aim we define the random variable $\xi_{f}^{(n)}(u)$ by

$$
\xi_{f}^{(n)}(u):=2 n\left(J_{f}^{(n)}(u)-\mathbb{E}\left[J_{f}^{(n)}(u)\right]\right) .
$$

First we compute the asymptotic variance of $\xi_{f}^{(n)}(u)$ as $n$ goes to infinity, which is the object of the following proposition. The proof as well as the following remark very closely follow the ones given in [14, Proposition 2.1].

\subsubsection{Asymptotic variance for $\xi_{f}^{(n)}(u)$}

The functionals $\xi_{f}^{(n)}(u)$ are also a sum of orthogonal terms in $L^{2}(\Omega)$. This is a crucial fact for computing its variance. Using the Arcones inequality (see [7, Lemma 1, p. 2245]), we deduce the asymptotic variance of $\xi_{f}^{(n)}(u)$ as $T_{n}$ grows to $\mathbb{R}^{2}$, this variance depending on the level $u$ as follows.

Proposition 4.3. For $f: S^{1} \rightarrow \mathbb{R}$ a function in $\mathcal{B}_{1}$, we have the following convergence,

$$
\lim _{n \rightarrow+\infty} \operatorname{Var}\left[\xi_{f}^{(n)}(u)\right]=\Sigma_{f, f}(u),
$$

$\Sigma_{f, f}(u)$ being defined by

$$
\Sigma_{f, f}(u):=\sum_{q=1}^{+\infty} \sum_{\substack{\mathbf{k}, \mathbf{m} \in \mathbb{N}^{3} \\|\boldsymbol{k}|=|\boldsymbol{m}|=q}} a_{f}(\boldsymbol{k}, u) a_{f}(\boldsymbol{m}, u) R(\boldsymbol{k}, \boldsymbol{m}),
$$

where coefficients $a_{f}(\boldsymbol{k}, u)$ have been defined by equality (3.9), while $R(\boldsymbol{k}, \boldsymbol{m})$ is defined as

$$
R(\boldsymbol{k}, \boldsymbol{m}):=\int_{\mathbb{R}^{2}} \mathbb{E}\left[\widetilde{H}_{\boldsymbol{k}}(U(0)) \widetilde{H}_{\boldsymbol{m}}(U(v))\right] \mathrm{d} v .
$$

Remark 4.4. If $f$ is a function with constant sign, then $\Sigma_{f, f}(u)>0$.

Proof of Proposition 4.3. One has the following expansion in $L^{2}(\Omega)$,

$$
\xi_{f}^{(n)}(u)=\frac{1}{\sqrt{\sigma_{2}\left(T_{n}\right)}} \sum_{q=1}^{+\infty} \sum_{\substack{\boldsymbol{k} \in \mathbb{N}^{3} \\|\boldsymbol{k}|=q}} a_{f}(\boldsymbol{k}, u) \int_{T_{n}} \widetilde{H}_{\boldsymbol{k}}(U(t)) \mathrm{d} t .
$$

Indeed by using Theorem 3.5 it remains to establish that $\mathbb{E}\left[J_{f}^{(n)}(u)\right]=a_{f}(\mathbf{0}, u)$, where $\mathbf{0}:=(0,0,0) \in \mathbb{N}^{3}$. Thus it is enough to remark that $a(0, u)=\phi\left(\frac{u}{\sqrt{r_{z}(0)}}\right) \frac{1}{\sqrt{r_{z}(0)}}=$ $p_{X(0)}(u)$ and that $a_{f}(0,0)=\mathbb{E}\left[f\left(\frac{\nabla X(0)}{\|\nabla X(0)\|_{2}}\right)\|\nabla X(0)\|_{2}\right]$, since

$$
\|\nabla X(0)\|_{2}=\sqrt{\mu}\left\|P \Lambda \cdot\left(\begin{array}{c}
U_{1}(0) \\
U_{2}(0)
\end{array}\right)\right\|_{2}=\sqrt{\mu}\left\|\Lambda \cdot\left(\begin{array}{c}
U_{1}(0) \\
U_{2}(0)
\end{array}\right)\right\|_{2} .
$$

Proposition 3.10 gives the result. 
Since the random variable $\xi_{f}^{(n)}(u)$ is a centred one, using equality given in (3.10) and Mehler's formula (see Lemma 3.9 of Section 3.2), we obtain

$$
\operatorname{Var}\left[\xi_{f}^{(n)}(u)\right]=\mathbb{E}\left[\xi_{f}^{(n)}(u)\right]^{2}=\sum_{q=1}^{+\infty} \sum_{\substack{\boldsymbol{k}, \boldsymbol{m} \in \mathbb{N}^{3} \\|\boldsymbol{k}|=|\boldsymbol{m}|=q}} a_{f}(\boldsymbol{k}, u) a_{f}(\boldsymbol{m}, u) R_{n}(\boldsymbol{k}, \boldsymbol{m})
$$

with

$$
R_{n}(\boldsymbol{k}, \boldsymbol{m}):=\frac{1}{(2 n)^{2}} \int_{]-n, n\left[^{2}\right.} \int_{]-n, n\left[^{2}\right.} \mathbb{E}\left[\widetilde{H}_{\boldsymbol{k}}(U(s)) \widetilde{H}_{\boldsymbol{m}}(U(t))\right] \mathrm{d} s \mathrm{~d} t .
$$

Thus and since $U$ is a stationary process, we have

$$
R_{n}(\boldsymbol{k}, \boldsymbol{m})=\int_{]-2 n, 2 n\left[^{2}\right.} \mathbb{E}\left[\widetilde{H}_{\boldsymbol{k}}(U(0)) \widetilde{H}_{\boldsymbol{m}}(U(v))\right]\left(1-\frac{\left|v_{1}\right|}{2 n}\right)\left(1-\frac{\left|v_{2}\right|}{2 n}\right) \mathrm{d} v .
$$

Now by applying Lemma 3.9 to $X:=U(0)$ and $Y:=U(v)$, one has for $|\boldsymbol{k}|=|\boldsymbol{m}|$,

$$
\mathbb{E}\left[\widetilde{H}_{\boldsymbol{k}}(U(0)) \widetilde{H}_{\boldsymbol{m}}(U(v))\right]=\sum_{\substack{d_{i j} \geqslant 0 \\ \sum_{j} d_{i j}=k_{i} \\ \sum_{i} d_{i j}=m_{j}}} \boldsymbol{k} ! \boldsymbol{m} ! \prod_{1 \leqslant i, j \leqslant 3} \frac{\left(\Gamma_{i j}^{U}(v)\right)^{d_{i j}}}{d_{i j} !},
$$

where

$$
\Gamma_{i j}^{U}(v):=\mathbb{E}\left[U_{i}(0) U_{j}(v)\right]
$$

Since

$$
\begin{aligned}
\Gamma^{U}(v) & =\left(\Gamma_{i j}^{U}(v)\right)_{1 \leqslant i, j \leqslant 3} \\
& =\left(\begin{array}{cc}
-\frac{1}{\mu} P^{t}\left(\frac{\partial^{2} r_{z}}{\partial v_{i} \partial v_{j}}(A . v)\right)_{1 \leqslant i, j \leqslant 2} P & -\frac{1}{\sqrt{\mu r_{z}(0)}} P^{t}\left(\frac{\partial r_{z}}{\partial v_{i}}(A . v)\right)_{1 \leqslant i \leqslant 2} \\
-\frac{1}{\sqrt{\mu r_{z}(0)}}\left(\frac{\partial r_{z}}{\partial v_{i}}(A . v)\right)_{1 \leqslant i \leqslant 2}^{t} P & \frac{r_{z}(A . v)}{r_{z}(0)}
\end{array}\right),
\end{aligned}
$$

we have for any $v \in \mathbb{R}^{2}$,

$$
\sup _{1 \leqslant i, j \leqslant 3}\left|\Gamma_{i j}^{U}(v)\right| \leqslant \mathbf{L} \Psi(A . v)
$$

where the function $\Psi$ has been introduced in Section 2.2 and $\mathbf{L}$ is some positive constant. Hence, for $|\boldsymbol{k}|=|\boldsymbol{m}|=q$, with $q \in \mathbb{N}^{\star}$,

$$
\left|\mathbb{E}\left[\widetilde{H}_{\boldsymbol{k}}(U(0)) \widetilde{H}_{\boldsymbol{m}}(U(v))\right]\right| \leqslant \mathbf{L}^{\prime} \Psi^{q}(A . v),
$$

where $\mathbf{L}^{\prime}$ is some constant depending on $q$.

By the covariance assumption $\mathbf{H}_{\Psi}$ previously stated in Section $2.2, \Psi \in L^{1}\left(\mathbb{R}^{2}\right)$ and since $\Psi$ is bounded then $\Psi^{q}(A \cdot) \in L^{1}\left(\mathbb{R}^{2}\right)$. We can apply the Lebesgue convergence theorem and obtain, for $\boldsymbol{k}, \boldsymbol{m} \in\left(\mathbb{N}^{3}\right)^{\star}$,

$$
\lim _{n \rightarrow+\infty} R_{n}(\boldsymbol{k}, \boldsymbol{m})=R(\boldsymbol{k}, \boldsymbol{m}):=\int_{\mathbb{R}^{2}} \mathbb{E}\left[\widetilde{H}_{\boldsymbol{k}}(U(0)) \widetilde{H}_{\boldsymbol{m}}(U(v))\right] \mathrm{d} v
$$

Now turning back to (4.4), we write

$$
\operatorname{Var}\left[\xi_{f}^{(n)}(u)\right]=\sum_{q=1}^{+\infty} V_{q}^{(n)}(u)
$$


and according to what we have just seen, for all $q \in \mathbb{N}^{\star}$,

$$
V_{q}(u):=\lim _{n \rightarrow+\infty} V_{q}^{(n)}(u)=\sum_{\substack{\boldsymbol{k}, \boldsymbol{m} \in \mathbb{N}^{3} \\|\boldsymbol{k}|=|\boldsymbol{m}|=q}} a_{f}(\boldsymbol{k}, u) a_{f}(\boldsymbol{m}, u) R(\boldsymbol{k}, \boldsymbol{m}) .
$$

Note that for any $q, V_{q}^{(n)}(u) \geqslant 0$ and so $V_{q}(u)$.

Thus, if we prove that $\lim _{Q \rightarrow+\infty} \sup _{n} \sum_{q=Q+1}^{+\infty} V_{q}^{(n)}(u)=0$, Fatou's lemma implies that $\lim _{Q \rightarrow+\infty} \sum_{q=Q+1}^{+\infty} V_{q}(u)=0$. Thus $\left(\sum_{q=1}^{Q} V_{q}(u)\right)_{Q}$ is an upper bounded increasing sequence and consequently a converging sequence, that is the series $\Sigma_{f, f}(u):=\sum_{q=1}^{+\infty} V_{q}(u)$ will be convergent. Also the first convergence will imply that $\operatorname{Var}\left[\xi_{f}^{(n)}(u)\right]$ tends to $\Sigma_{f, f}(u)$. The proof of Proposition 4.3 will be completed. Thus let us prove this convergence via a lemma.

Lemma 4.5. For $q, n \in \mathbb{N}^{\star}$, let

$$
V_{q}^{(n)}(u):=\sum_{\substack{\boldsymbol{k}, \boldsymbol{m} \in \mathbb{N}^{3} \\|\boldsymbol{k}|=|\boldsymbol{m}|=q}} a_{f}(\boldsymbol{k}, u) a_{f}(\boldsymbol{m}, u) R_{n}(\boldsymbol{k}, \boldsymbol{m}),
$$

with $f: S^{1} \rightarrow \mathbb{R}$ a function in $\mathcal{B}_{1}$. One has the following convergence

$$
\lim _{Q \rightarrow+\infty} \sup _{n \geqslant 1} \sum_{q=Q+1}^{+\infty} V_{q}^{(n)}(u)=0 .
$$

Proof of Lemma 4.5. First, let us remark that the convergence in (4.8) is equivalent to the following one:

$$
\lim _{Q \rightarrow+\infty} \operatorname{Var}\left[\pi_{Q}\left(\xi_{f}^{(n)}(u)\right)\right]=0
$$

uniformly with respect to $n$, where $\pi_{Q}$ stands for the projection onto the chaos of strictly upper order in $Q$.

For the sake of simplicity of writing, let us note $V_{n, Q}:=\operatorname{Var}\left[\pi_{Q}\left(\xi_{f}^{(n)}(u)\right)\right]$. Let $s \in \mathbb{R}^{2}$ and $\theta_{s}$ be the shift operator associated with the field $X$, that is, $\theta_{s} X:=X_{s+}$. Let us also introduce the set of indices $I_{n}:=\left[-n, n\left[^{2} \cap \mathbb{Z}^{2}\right.\right.$, clearly we have

$$
\pi_{Q}\left(\xi_{f}^{(n)}(u)\right)=\frac{1}{2 n} \sum_{s \in I_{n}} \theta_{s}\left(\pi_{Q}\left(\xi_{f, 1}(u)\right)\right)
$$

where the random variable $\xi_{f, 1}(u)$ is

$$
\xi_{f, 1}(u):=\sum_{q=1}^{+\infty} \sum_{\substack{\boldsymbol{k} \in \mathbb{N}^{3} \\|\boldsymbol{k}|=q}} a_{f}(\boldsymbol{k}, u) \int_{] 0,1\left[^{2}\right.} \widetilde{H}_{\boldsymbol{k}}(U(t)) \mathrm{d} t
$$

The stationarity of $X$ leads to

$$
V_{n, Q}=\left(\frac{1}{2 n}\right)^{2} \sum_{s \in I_{2 n}} \alpha_{s}(n) \mathbb{E}\left[\pi_{Q}\left(\xi_{f, 1}(u)\right) \theta_{s}\left(\pi_{Q}\left(\xi_{f, 1}(u)\right)\right)\right],
$$

where $\alpha_{s}(n):=\operatorname{card}\left\{t \in I_{n}, t-s \in I_{n}\right\}$.

Obviously, one has $\alpha_{s}(n) \leqslant(2 n)^{2}$.

Now, on the one hand, by the covariance assumption $\mathbf{H}_{\Psi}$ made in Section 2.2, 
$\lim _{\|x\|_{2} \rightarrow+\infty} \Psi(x)=0$, and since the eigenvalues of $A$ are strictly positive one also has $\lim _{\|x\|_{2} \rightarrow+\infty} \Psi(A . x)=0$.

On the other hand, let $0<\rho<1$ such that

$$
\rho \mathbf{A}(u)<1 \text { where } \mathbf{A}(u):=2 \frac{u^{2}}{r_{z}(0)}+1,
$$

and $a>0$ such that $\|x\|_{2} \geqslant a$ implies

$$
3 \mathbf{L} \Psi(A x) \leqslant \rho<1
$$

where $\mathbf{L}$ is defined by (4.6).

We split $V_{n, Q}$ into $V_{n, Q}=V_{n, Q}^{(1)}+V_{n, Q}^{(2)}$, where in $V_{n, Q}^{(1)}$ the sum runs over the indices $s$ in $\left\{s \in I_{2 n},\|s\|_{\infty}<a+3\right\}$ and in $V_{n, Q}^{(2)}$ over $s$ in $\left\{s \in I_{2 n},\|s\|_{\infty} \geqslant a+3\right\},\|\cdot\|_{\infty}$ standing for the supremum norm. By Schwarz inequality and since $\alpha_{s}(n) \leqslant(2 n)^{2}$, using the stationarity of $X$ one has the following upper bound,

$$
\left|V_{n, Q}^{(1)}\right| \leqslant(2(a+3))^{2} \mathbb{E}\left[\pi_{Q}\left(\xi_{f, 1}(u)\right)\right]^{2},
$$

which goes to zero as $Q$ goes to infinity uniformly with respect to $n$, since adapting equality (3.10) to the present situation one can proved that $\lim _{Q \rightarrow+\infty} \mathbb{E}\left[\pi_{Q}\left(\xi_{f, 1}(u)\right)\right]^{2}=0$. We proved that $\lim _{Q \rightarrow+\infty} \sup _{n} V_{n, Q}^{(1)}=0$.

Now, let us consider the term $V_{n, Q}^{(2)}$.

$$
V_{n, Q}^{(2)}:=\left(\frac{1}{2 n}\right)^{2} \sum_{\substack{s \in I_{2 n} \\\|s\|_{\infty} \geqslant a+3}} \alpha_{s}(n) \mathbb{E}\left[\pi_{Q}\left(\xi_{f, 1}(u)\right) \theta_{s}\left(\pi_{Q}\left(\xi_{f, 1}(u)\right)\right)\right] .
$$

For $q \in \mathbb{N}^{\star}$, let us define function $F_{q}$ by

$$
F_{q}(x):=\sum_{\substack{\boldsymbol{k} \in \mathbb{N}^{3} \\|\boldsymbol{k}|=q}} a_{f}(\boldsymbol{k}, u) \widetilde{H}_{\boldsymbol{k}}(x), x \in \mathbb{R}^{3} .
$$

For $s \in I_{2 n}$ such that $\|s\|_{\infty} \geqslant a+3$,

$$
\begin{aligned}
& \mathbb{E}\left[\pi_{Q}\left(\xi_{f, 1}(u)\right) \theta_{s}\left(\pi_{Q}\left(\xi_{f, 1}(u)\right)\right)\right]= \\
& \sum_{q=Q+1}^{+\infty} \int_{] 0,1\left[^{2}\right.} \int_{] 0,1\left[^{2}\right.} \mathbb{E}\left[F_{q}(U(t)) F_{q}(U(s+v))\right] \mathrm{d} t \mathrm{~d} v .
\end{aligned}
$$

At this step of the proof we want to propose a bound for $\mathbb{E}\left[F_{q}(U(t)) F_{q}(U(s+v))\right], t, v \in$ ] $0,1\left[^{2}\right.$.

Note that if $\sum_{\substack{\boldsymbol{k} \in \mathbb{N}^{3} \\|\boldsymbol{k}|=q}} a_{f}^{2}(\boldsymbol{k}, u) \boldsymbol{k} !=0$, then $F_{q}(x)=0$ for all $x \in \mathbb{R}^{3}$ and a trivial bound is zero. So let us suppose that $\sum_{\substack{\boldsymbol{k} \in \mathbb{N}^{3} \\|\boldsymbol{k}|=q}} a_{f}^{2}(\boldsymbol{k}, u) \boldsymbol{k} ! \neq 0$.

We are going to apply Arcones inequality (see [7, Lemma 1 p. 2245]). By using notations of this lemma, we apply it to $f:=F_{q}$ and to $X=\left(X^{(j)}\right)_{1 \leqslant j \leqslant 3}:=U(t)$ and $Y=\left(Y^{(k)}\right)_{1 \leqslant k \leqslant 3}:=U(s+v)$, with $d=3$, such that $r^{(j, k)}=\mathbb{E}\left[X^{(j)} Y^{(k)}\right]:=\Gamma_{j k}^{U}(s-t+v)$, $\Gamma^{U}$ being defined in (4.5).

Now by using inequalities given in (4.6) and (4.10),

$$
\begin{array}{r}
\psi:=\left(\sup _{1 \leqslant j \leqslant 3} \sum_{k=1}^{3}\left|r^{(j, k)}\right|\right) \vee\left(\sup _{1 \leqslant k \leqslant 3} \sum_{j=1}^{3}\left|r^{(j, k)}\right|\right) \\
\leqslant 3 \mathbf{L} \Psi(A .(s-t+v)) \leqslant \rho<1 .
\end{array}
$$


It remains to verify that $F_{q}$ function on $\mathbb{R}^{3}$ has finite second moment and rank $q$. In the first place by Lemma 3.9 given in Section 3.2 one has

$$
\mathbb{E}\left[F_{q}(X)\right]^{2}=\mathbb{E}\left[F_{q}(U(t))\right]^{2}=\sum_{\substack{\boldsymbol{k} \in \mathbb{N}^{3} \\|\boldsymbol{k}|=q}} a_{f}^{2}(\boldsymbol{k}, u) \boldsymbol{k} !<+\infty
$$

In the second place and since $\sum_{\substack{\boldsymbol{k} \in \mathbb{N}^{3} \\|\boldsymbol{k}|=q}} a_{f}^{2}(\boldsymbol{k}, u) \boldsymbol{k} ! \neq 0$, this last equality ensures that rank $F_{q} \leqslant q$. Furthermore let $\boldsymbol{m} \in \mathbb{N}^{3}$ such that $\mathbb{E}\left[F_{q}(X) \widetilde{H}_{\boldsymbol{m}}(X)\right] \neq 0$. By Lemma 3.9, $\mathbb{E}\left[F_{q}(X) \widetilde{H}_{\boldsymbol{m}}(X)\right]=\sum_{\substack{\boldsymbol{k} \in \mathbb{N}^{3} \\|\boldsymbol{k}|=q}} a_{f}(\boldsymbol{k}, u) \boldsymbol{k} ! \mathbb{1}_{|\boldsymbol{k}|=|\boldsymbol{m}|}$, which implies $|\boldsymbol{m}|=q$ and $\operatorname{rank} F_{q}=q$.

Thus we have all the ingredients to apply Arcones inequality. For $q \geqslant 1$, using inequality given in (4.11) we get the bound

$$
\begin{aligned}
\mathbb{E}\left[F_{q}(U(t)) F_{q}(U(s+v))\right] \leqslant \psi^{q} \mathbb{E}\left[F_{q}(U(t))\right]^{2} & \\
& \leqslant \rho^{q-1}(3 \mathbf{L}) \Psi(A .(s-t+v))\left(\sum_{\substack{\boldsymbol{k} \in \mathbb{N}^{3} \\
|\boldsymbol{k}|=q}} a_{f}^{2}(\boldsymbol{k}, u) \boldsymbol{k} !\right) .
\end{aligned}
$$

As already pointed out in Remark 3.6, the series $\sum_{\boldsymbol{k} \in \mathbb{N}^{3}} a_{f}^{2}(\boldsymbol{k}, u) \boldsymbol{k} !=+\infty$, so that we have to tread carefully in what follows.

Finally and since $\alpha_{s}(n) \leqslant(2 n)^{2}$, one has

$$
\left|V_{n, Q}^{(2)}\right| \leqslant \mathbf{C} \sum_{q=Q+1}^{+\infty} \sum_{\substack{\boldsymbol{k} \in \mathbb{N}^{3} \\|\boldsymbol{k}|=q}} \rho^{q-1} a_{f}^{2}(\boldsymbol{k}, u) \boldsymbol{k} ! \sum_{s \in I_{2 n}} \int_{] 0,1\left[^{2}\right.} \int_{] 0,1\left[^{2}\right.} \Psi(A .(s-t+v)) \mathrm{d} t \mathrm{~d} v .
$$

Using that

$$
\begin{aligned}
\sum_{s \in I_{2 n}} \int_{] 0,1\left[^{2}\right.} \int_{] 0,1\left[^{2}\right.} \Psi(A .(s-t+v)) \mathrm{d} t \mathrm{~d} v \leqslant \int_{\mathbb{R}^{2}} \Psi(A . v) \mathrm{d} v & \\
& \leqslant \mathbf{C} \int_{\mathbb{R}^{2}} \Psi(v) \mathrm{d} v<+\infty
\end{aligned}
$$

last finiteness providing from assumption $\mathbf{H}_{\Psi}$ made in Section 2.2, one has

$$
\left|V_{n, Q}^{(2)}\right| \leqslant \mathbf{C} \sum_{q=Q+1}^{+\infty} \sum_{\substack{\boldsymbol{k} \in \mathbb{N}^{3} \\|\boldsymbol{k}|=q}} \rho^{q-1} a_{f}^{2}(\boldsymbol{k}, u) \boldsymbol{k} ! .
$$

To conclude the proof of this lemma we just have to check that

$$
\sum_{q=1}^{+\infty} \sum_{\substack{\boldsymbol{k} \in \mathbb{N}^{3} \\|\boldsymbol{k}|=q}} \rho^{q-1} a_{f}^{2}(\boldsymbol{k}, u) \boldsymbol{k} !<+\infty
$$

Now remember that for $\boldsymbol{k}=\left(k_{i}\right)_{1 \leqslant i \leqslant 3} \in \mathbb{N}^{3}$,

$$
a_{f}(\boldsymbol{k}, u)=a_{f}\left(k_{1}, k_{2}\right) a\left(k_{3}, u\right),
$$

with $a_{f}\left(k_{1}, k_{2}\right)$ and $a\left(k_{3}, u\right)$ respectively defined by equalities (3.6) and (3.8).

On the one hand, since the function

$$
h:\left(y_{1}, y_{2}\right) \mapsto f\left(\frac{P \Lambda \cdot\left(\begin{array}{l}
y_{1} \\
y_{2}
\end{array}\right)}{\sqrt{\lambda_{1}^{2} y_{1}^{2}+\lambda_{2}^{2} y_{2}^{2}}}\right) \sqrt{\lambda_{1}^{2} y_{1}^{2}+\lambda_{2}^{2} y_{2}^{2}}
$$


is such that $h \in L\left(\mathbb{R}^{2}, \phi_{2}(y) \mathrm{d} y\right)$, we deduce that,

$$
\sum_{k_{1}, k_{2} \in \mathbb{N}} a_{f}^{2}\left(k_{1}, k_{2}\right) k_{1} ! k_{2} !<+\infty .
$$

On the other hand by using the expression of Hermite's polynomials given in Appendix A by (A.3) and (A.4), for all $k \in \mathbb{N}$ and for all $x \in \mathbb{R}$ we get the bound

$$
H_{k}^{2}(x) \leqslant(k+1) !\left(2 x^{2}+1\right)^{k},
$$

so that for all $\boldsymbol{k} \in \mathbb{N}^{3}$ such that $|\boldsymbol{k}|=q$ and remembering that $\mathbf{A}(u)$ has been defined in (4.9), one has

$$
k_{3} ! a^{2}\left(k_{3}, u\right) \leqslant \mathbf{C}\left(k_{3}+1\right) \mathbf{A}^{k_{3}}(u) \leqslant \mathbf{C}(q+1) \mathbf{A}^{q}(u) .
$$

Thus by inequality (4.12), finally one obtains

$$
\sum_{q=1}^{+\infty} \sum_{\substack{\boldsymbol{k} \in \mathbb{N}^{3} \\|\boldsymbol{k}|=q}} \rho^{q-1} a_{f}^{2}(\boldsymbol{k}, u) \boldsymbol{k} ! \leqslant \mathbf{C} \sum_{q=1}^{+\infty} \rho^{q-1}(q+1)^{2} \mathbf{A}^{q}(u)<+\infty,
$$

last finiteness providing from inequality (4.9).

This yields Lemma 4.5.

Proposition 4.3 ensues.

Proof of Remark 4.4. Remark ensues from the following argumentation.

We have seen in the proof of Proposition 4.3 that $\Sigma_{f, f}(u)=\sum_{q=1}^{+\infty} V_{q}(u)$, with

$$
V_{q}(u)=\sum_{\substack{\mathbf{k}, \mathbf{m} \in \mathbb{N}^{3} \\|\boldsymbol{k}|=|\boldsymbol{m}|=q}} a_{f}(\boldsymbol{k}, u) a_{f}(\boldsymbol{m}, u) R(\boldsymbol{k}, \boldsymbol{m}) \geqslant 0,
$$

for all $q \geqslant 1$. Thus

$$
\Sigma_{f, f}(u) \geqslant V_{1}(u)+V_{2}(u) .
$$

By using Lemma 3.9 and the inversion formula, a computation gives that for $|\boldsymbol{k}|=|\boldsymbol{m}|=1$, $R(\boldsymbol{k}, \boldsymbol{m})=0$ except when $\boldsymbol{k}=\boldsymbol{m}=(0,0,1)$ and in this case one has

$$
R((0,0,1),(0,0,1))=\frac{1}{r_{x}(0)} \int_{\mathbb{R}^{2}} r_{x}(v) \mathrm{d} v=(2 \pi)^{2} \frac{f_{z}(0)}{\lambda_{1} \lambda_{2} r_{z}(0)} .
$$

Thus

$$
V_{1}(u)=a_{f}^{2}(0,0) \frac{u^{2}}{r_{z}^{2}(0)} \phi^{2}\left(\frac{u}{\sqrt{r_{z}(0)}}\right)(2 \pi)^{2} \frac{f_{z}(0)}{\lambda_{1} \lambda_{2} r_{z}(0)}>0,
$$

if $u \neq 0$, since $f$ is supposed to have constant sign and $f_{z}(0)>0$ (see Remark 2.11 given in Section 2.2).

Using arguments similar to the previous ones, the fact that $\int_{\mathbb{R}^{2}} f_{z}(t)\|t\|_{2}^{2} \mathrm{~d} t<+\infty$ (see Remark 2.12) and Parseval equality, straightforward calculations provide that

$$
\begin{aligned}
V_{2}(u)= & 2 \times(2 \pi)^{2} \int_{\mathbb{R}^{2}} f_{x}^{2}(t) \times\left[a _ { f } ( ( 1 , 1 , 0 ) , u ) \left(d_{11} d_{21} t_{1}^{2}\right.\right. \\
& \left.+\left(d_{11} d_{22}+d_{12} d_{21}\right) t_{1} t_{2}+d_{12} d_{22} t_{2}^{2}\right)+a_{f}((1,0,1), u) \frac{1}{\sqrt{r_{z}(0)}}\left(d_{11} t_{1}+d_{12} t_{2}\right) \\
& +a_{f}((0,1,1), u) \frac{1}{\sqrt{r_{z}(0)}}\left(d_{21} t_{1}+d_{22} t_{2}\right)+a_{f}((2,0,0), u)\left(d_{11} t_{1}+d_{12} t_{2}\right)^{2} \\
& \left.+a_{f}((0,2,0), u)\left(d_{21} t_{1}+d_{22} t_{2}\right)^{2}+a_{f}((0,0,2), u) \frac{1}{r_{z}(0)}\right]^{2} \mathrm{~d} t \geqslant 0,
\end{aligned}
$$


where $\left(d_{i j}\right)_{1 \leqslant i, j \leqslant 2}=D:=\frac{1}{\sqrt{\mu}} \Lambda^{-1} P^{t}$.

Remark 4.6. Note that in the case where the process $X$ is isotropic our result contains that of Kratz and León [20, Theorem 3].

Since $\operatorname{det}(D) \neq 0$, one gets the following equivalence:

$$
\left(V_{2}(u)=0\right) \Longleftrightarrow\left(a_{f}(\boldsymbol{k}, u)=0, \text { for all } \boldsymbol{k} \in \mathbb{N}^{3} \text { such that }|\mathbf{k}|=2\right)
$$

In particular, since $f$ has a constant sign, $a_{f}(0,0) \neq 0$ so that $a_{f}((0,0,2), 0) \neq 0$ and $V_{2}(0)>0$.

Finally we proved that for $u \neq 0, \Sigma_{f, f}(u) \geqslant V_{1}(u)+V_{2}(u) \geqslant V_{1}(u)>0$ and for $u=0$, $\Sigma_{f, f}(0) \geqslant V_{1}(0)+V_{2}(0) \geqslant V_{2}(0)>0$.

The proof of Remark 4.4 is completed.

Now, we have got all the tools to prove that the random variable $\xi_{f}^{(n)}(u)$ converges in law as $n$ tends to infinity to a centred Gaussian variable with finite variance $\Sigma_{f, f}(u)$ given by (4.2), see Theorem 4.7. The proof we give for this theorem, is inspired by the one presented in [14, Proposition 2.4].

\subsubsection{General level functionals viewed into the Wiener-Itô chaos}

Using the Peccati and Tudor theorem (see [26]), we obtain the following theorem.

Theorem 4.7. For $f: S^{1} \rightarrow \mathbb{R}$ a function in $\mathcal{B}_{1}$, we have the following convergence,

$$
\xi_{f}^{(n)}(u) \underset{n \rightarrow+\infty}{\stackrel{L a w}{\longrightarrow}} \mathcal{N}\left(0 ; \Sigma_{f, f}(u)\right) .
$$

Remark 4.8. For example if $f \equiv 1$, we find that the normalized centred curve length converges in law to a non degenerate Gaussian random variable.

Note that for all real numbers $a$ and $b$ and for all functions, $f_{1}$ and $f_{2}: S^{1} \rightarrow \mathbb{R}$, belonging to $\mathcal{B}_{1}$, one has $\xi_{a f_{1}+b f_{2}}^{(n)}(u)=a \xi_{f_{1}}^{(n)}(u)+b \xi_{f_{2}}^{(n)}(u)$.

By generalizing the definition given in (4.1), we define the following functional. For $k \in \mathbb{N}^{\star}$ and $\vec{f}:=\left(f_{1}, f_{2}, \cdots, f_{k}\right): S^{1} \rightarrow \mathbb{R}^{k}$ function belonging to $\mathcal{B}_{k}$, let

$$
\xi_{\vec{f}}^{(n)}(u):=2 n\left(J_{\vec{f}}^{(n)}(u)-\mathbb{E}\left[J_{\vec{f}}^{(n)}(u)\right]\right)
$$

where

$$
J_{\vec{f}}^{(n)}(u)-\mathbb{E}\left[J_{\vec{f}}^{(n)}(u)\right]:=\left(J_{f_{i}}^{(n)}(u)-\mathbb{E}\left[J_{f_{i}}^{(n)}(u)\right]\right)_{1 \leqslant i \leqslant k}
$$

We define

$$
\Sigma_{f_{i}, f_{j}}(u):=\sum_{q=1}^{+\infty} \sum_{\substack{\boldsymbol{k}, \boldsymbol{m} \in \mathbb{N}^{3} \\|\boldsymbol{k}|=|\boldsymbol{m}|=q}} a_{f_{i}}(\boldsymbol{k}, u) a_{f_{j}}(\boldsymbol{m}, u) R(\boldsymbol{k}, \boldsymbol{m}),
$$

where $R(\boldsymbol{k}, \boldsymbol{m})$ is defined by (4.3), and

$$
\Sigma_{\vec{f}}(u):=\left(\Sigma_{f_{i}, f_{j}}(u)\right)_{1 \leqslant i, j \leqslant k} .
$$

By Cramer-Wold device we readily get Corollary 4.9.

Corollary 4.9. For all $k \in \mathbb{N}^{\star}$ and all function $\vec{f}:=\left(f_{1}, \cdots, f_{k}\right): S^{1} \rightarrow \mathbb{R}^{k}$ belonging to $\mathcal{B}_{k}$, one has

$$
\xi_{\vec{f}}^{(n)}(u) \underset{n \rightarrow+\infty}{\stackrel{L a w}{\longrightarrow}} \mathcal{N}\left(0 ; \Sigma_{\vec{f}}(u)\right)
$$


Proof of Theorem 4.7. First, let $Q$ a fixed integer in $\mathbb{N}^{\star}$ and let us consider the projection of the random variable $\xi_{f}^{(n)}(u)$ onto the first $Q$ chaos in $L^{2}(\Omega)$ that is

$$
\pi^{Q}\left(\xi_{f}^{(n)}(u)\right):=\frac{1}{2 n} \sum_{q=1}^{Q} \sum_{\substack{\boldsymbol{k} \in \mathbb{N}^{3} \\|\boldsymbol{k}|=q}} a_{f}(\boldsymbol{k}, u) \int_{T_{n}} \widetilde{H}_{\boldsymbol{k}}(U(t)) \mathrm{d} t .
$$

We will show the asymptotic normality of this sequence as $n$ tends to infinity. For this purpose and in order to apply the Peccati and Tudor theorem (see [26, Theorem 1]), we will give an expansion of this random variable into the Wiener-Itô chaos of order less or equal to $Q$.

To this end, remember that in Section 3.2 for any $t \in \mathbb{R}^{2}$ one has defined the 3-dimensional standard Gaussian vector $U(t)$ as

$$
U(t)=\int_{\mathbb{R}^{2}} e^{i\langle t, \lambda\rangle} \sqrt{f_{x}(\lambda)} \Delta^{-1} . \nu(\lambda) \mathrm{d} W(\lambda)=\left(U_{i}(t)\right)_{1 \leqslant i \leqslant 3}
$$

where $W$ stands for the standard Brownian motion and for any $\lambda=\left(\lambda_{i}\right)_{1 \leqslant i \leqslant 2}$ in $\mathbb{R}^{2}$,

$$
\nu(\lambda)=\left(i \lambda_{1}, i \lambda_{2}, 1\right)^{t}
$$

In what follows, for any $t \in \mathbb{R}^{2}$ and $j=1,2,3$, we denote by $\varphi_{t, j}$ the square integrable map on $\mathbb{R}^{2}$ such that,

$$
U_{j}(t)=\int_{\mathbb{R}^{2}} \varphi_{t, j}(\lambda) \mathrm{d} W(\lambda)
$$

Since $\left(\varphi_{t, j}\right)_{1 \leqslant j \leqslant 3}$ is an orthonormal system in $L^{2}\left(\mathbb{R}^{2}\right)$, using Itô's formula (see Major [23, Theorem 4.2 p. 30]), for fixed $\boldsymbol{k}=\left(k_{i}\right)_{1 \leqslant i \leqslant 3} \in \mathbb{N}^{3}$ such that $|\boldsymbol{k}|=q$,

$$
\begin{aligned}
\widetilde{H}_{\boldsymbol{k}}(U(t)) & =\prod_{j=1}^{3} H_{k_{j}}\left(U_{j}(t)\right) \\
& =\int_{\mathbb{R}^{2 q}} \varphi_{t, 1}^{\otimes k_{1}} \otimes \varphi_{t, 2}^{\otimes k_{2}} \otimes \varphi_{t, 3}^{\otimes k_{3}}\left(\lambda_{1}, \ldots, \lambda_{q}\right) \mathrm{d} W\left(\lambda_{1}\right) \cdots \mathrm{d} W\left(\lambda_{q}\right) \\
& =I_{q}\left(\varphi_{t, 1}^{\otimes k_{1}} \otimes \varphi_{t, 2}^{\otimes k_{2}} \otimes \varphi_{t, 3}^{\otimes k_{3}}\right),
\end{aligned}
$$

where $I_{q}$ stands for the Wiener-Itô integral of order $q$.

We shall use notations introduced in Slud [30].

For each $q \geqslant 1$, let consider $L_{\text {sym }}^{2}\left(\left(\mathbb{R}^{2}\right)^{q}\right)$ the complex Hilbert-space

$$
\begin{aligned}
& L_{\mathrm{sym}}^{2}\left(\left(\mathbb{R}^{2}\right)^{q}\right):=\left\{f_{q} \in L^{2}\left(\left(\mathbb{R}^{2}\right)^{q}\right), \text { such that for all } x \in\left(\mathbb{R}^{2}\right)^{q},\right. \\
& \left.f_{q}(x)=\overline{f_{q}(-x)}, f_{q}\left(x_{1}, \ldots, x_{q}\right)=f_{q}\left(x_{\pi(1)}, \ldots, x_{\pi(q)}\right), \forall \pi \in S_{q}\right\},
\end{aligned}
$$

where $S_{q}$ denotes the symmetric group of permutations of $\{1, \ldots, q\}$.

For $f_{q} \in L^{2}\left(\left(\mathbb{R}^{2}\right)^{q}\right)$, we denote by $\operatorname{sym}\left(f_{q}\right)$ the symmetrization of $f_{q}$, that is for $x_{1}, \ldots, x_{q} \in$ $\mathbb{R}^{2}$,

$$
\operatorname{sym}\left(f_{q}\right)\left(x_{1}, \ldots, x_{q}\right):=\frac{1}{q !} \sum_{\pi \in S_{q}} f_{q}\left(x_{\pi(1)}, \ldots, x_{\pi(q)}\right)
$$

Observe that for $f_{q} \in L^{2}\left(\left(\mathbb{R}^{2}\right)^{q}\right)$ such that for all $x \in\left(\mathbb{R}^{2}\right)^{q}, f_{q}(x)=\overline{f_{q}(-x)}$, one has

$$
I_{q}\left(f_{q}\right)=I_{q}\left(\operatorname{sym}\left(f_{q}\right)\right) .
$$


For $q \geqslant 1, f_{q} \in L_{\mathrm{sym}}^{2}\left(\left(\mathbb{R}^{2}\right)^{q}\right)$ and $p=1, \ldots, q$, we will write $f_{q} \otimes_{p} f_{q}$ for the $p$-th contraction of $f_{q}$ defined as

$$
\begin{array}{r}
f_{q} \otimes_{p} f_{q}\left(\lambda_{1}, \ldots, \lambda_{2 q-2 p}\right):=\int_{\left(\mathbb{R}^{2}\right)^{p}} f_{q}\left(\lambda_{1}, \ldots, \lambda_{q-p}, x_{1}, \ldots, x_{p}\right) \\
f_{q}\left(\lambda_{q-p+1}, \ldots, \lambda_{2 q-2 p},-x_{1}, \ldots,-x_{p}\right) \mathrm{d} x_{1} \cdots \mathrm{d} x_{p} .
\end{array}
$$

Using the property of $I_{q}$ given in (4.15), the random variable of interest can be written as

$$
\pi^{Q}\left(\xi_{f}^{(n)}(u)\right)=\sum_{q=1}^{Q} I_{q}\left(f_{q}^{(n)}\right)
$$

where for $n, q \in \mathbb{N}^{\star}$

$$
f_{q}^{(n)}:=\frac{1}{2 n} \sum_{\substack{\boldsymbol{k} \in \mathbb{N}^{3} \\|\boldsymbol{k}|=q}} a_{f}(\boldsymbol{k}, u) \int_{T_{n}} \operatorname{sym}\left(\varphi_{t, 1}^{\otimes k_{1}} \otimes \varphi_{t, 2}^{\otimes k_{2}} \otimes \varphi_{t, 3}^{\otimes k_{3}}\right) \mathrm{d} t .
$$

We symmetrized the function $f_{q}^{(n)}$ with the aim of applying [26, Theorem 1].

Symmetrizing the function complicates a lot the calculations in the study of the contractions. So we are going to write function $f_{q}^{(n)}$ in another way.

For $\boldsymbol{k}=\left(k_{i}\right)_{1 \leqslant i \leqslant 3}$ such that $|\boldsymbol{k}|=q$, we define

$$
\mathcal{A}_{\boldsymbol{k}}:=\left\{m=\left(m_{1}, \ldots, m_{q}\right) \in\{1,2,3\}^{q}, \forall i=1,2,3, \sum_{j=1}^{q} \mathbb{1}_{\{i\}}\left(m_{j}\right)=k_{i}\right\},
$$

one has $\operatorname{card}\left\{\mathcal{A}_{\boldsymbol{k}}\right\}=\boldsymbol{k} ! / q$ !. Let us remark that the sets $\left(\mathcal{A}_{\boldsymbol{k}}\right)_{\boldsymbol{k} \in \mathbb{N}^{3},|\boldsymbol{k}|=q}$ provide a partition of $\{1,2,3\}^{q}$.

With these notations one has

$$
\sum_{\substack{\boldsymbol{k} \in \mathbb{N}^{3} \\|\boldsymbol{k}|=q}} a_{f}(\boldsymbol{k}, u) \operatorname{sym}\left(\varphi_{t, 1}^{\otimes k_{1}} \otimes \varphi_{t, 2}^{\otimes k_{2}} \otimes \varphi_{t, 3}^{\otimes k_{3}}\right)=
$$

$$
\operatorname{sym}\left(\sum_{\substack{\boldsymbol{k} \in \mathbb{N}^{3} \\|\boldsymbol{k}|=q}} \sum_{m \in \mathcal{A}_{\boldsymbol{k}}} \frac{a_{f}(\boldsymbol{k}, u)}{\operatorname{card}\left(\mathcal{A}_{\boldsymbol{k}}\right)} \varphi_{t, m_{1}} \ldots \varphi_{t, m_{q}}\right) .
$$

Since $\left(\mathcal{A}_{\boldsymbol{k}}\right)_{\boldsymbol{k} \in \mathbb{N}^{3},|\boldsymbol{k}|=q}$ provides a partition of $\{1,2,3\}^{q}$, then for all $m \in\{1,2,3\}^{q}, \exists ! \boldsymbol{k} \in \mathbb{N}^{3}$ such that $|\boldsymbol{k}|=q$ and $m \in \mathcal{A}_{\boldsymbol{k}}$. So for fixed $m \in\{1,2,3\}^{q}$, let $b_{f}(m, u):=\frac{a_{f}(\boldsymbol{k}, u)}{\operatorname{card}\left(\mathcal{A}_{\boldsymbol{k}}\right)}$. Thus

$$
\begin{gathered}
\sum_{\substack{\boldsymbol{k} \in \mathbb{N}^{3} \\
|\boldsymbol{k}|=q}} a_{f}(\boldsymbol{k}, u) \operatorname{sym}\left(\varphi_{t, 1}^{\otimes k_{1}} \otimes \varphi_{t, 2}^{\otimes k_{2}} \otimes \varphi_{t, 3}^{\otimes k_{3}}\right) \\
=\operatorname{sym}\left(\sum_{m \in\{1,2,3\}^{q}} b_{f}(m, u) \varphi_{t, m_{1}} \cdots \varphi_{t, m_{q}}\right) \\
=\sum_{m \in\{1,2,3\}^{q}} b_{f}(m, u) \varphi_{t, m_{1}} \cdots \varphi_{t, m_{q}}
\end{gathered}
$$

since $m \mapsto b_{f}(m, u)$ is symmetric on $\{1,2,3\}^{q}$.

Finally the random variable $\pi^{Q}\left(\xi_{f}^{(n)}(u)\right)$ can be written as

$$
\pi^{Q}\left(\xi_{f}^{(n)}(u)\right)=\sum_{q=1}^{Q} I_{q}\left(f_{q}^{(n)}\right)
$$


where for $n, q \in \mathbb{N}^{\star}$

$$
f_{q}^{(n)}:=\frac{1}{2 n} \int_{]-n, n\left[^{2}\right.} \sum_{m \in\{1,2,3\}^{q}} b_{f}(m, u) \varphi_{t, m_{1}} \cdots \varphi_{t, m_{q}} \mathrm{~d} t
$$

that ends our first objective.

To obtain convergence of $\pi^{Q}\left(\xi_{f}^{(n)}(u)\right)$, we use [26, Theorem 1]. Convergence in Proposition 4.3 gives the required conditions appearing at the beginning of this latter theorem. So we just verify condition (i) in proving the following lemma.

Lemma 4.10. For fixed integers $q$ and $p$ such that $q \geqslant 2$ and $p=1, \ldots, q-1$,

$$
\begin{array}{r}
\lim _{n \rightarrow+\infty} \int_{\left(\mathbb{R}^{2}\right)^{2(q-p)}}\left|f_{q}^{(n)} \otimes_{p} f_{q}^{(n)}\left(\lambda_{1}, \ldots, \lambda_{q-p}, \mu_{1}, \ldots, \mu_{q-p}\right)\right|^{2} \\
\mathrm{~d} \lambda_{1} \ldots \mathrm{d} \lambda_{q-p} \mathrm{~d} \mu_{1} \ldots \mathrm{d} \mu_{q-p}=0 .
\end{array}
$$

Proof of Lemma 4.10. Let

$$
\begin{array}{r}
C_{n}:=\int_{\left(\mathbb{R}^{2}\right)^{2(q-p)}}\left|f_{q}^{(n)} \otimes_{p} f_{q}^{(n)}\left(\lambda_{1}, \ldots, \lambda_{q-p}, \mu_{1}, \ldots, \mu_{q-p}\right)\right|^{2} \\
\mathrm{~d} \lambda_{1} \ldots \mathrm{d} \lambda_{q-p} \mathrm{~d} \mu_{1} \ldots \mathrm{d} \mu_{q-p} .
\end{array}
$$

Straightforwards calculations show that

$$
\begin{gathered}
C_{n}=\left(\frac{1}{2 n}\right)^{4} \int_{(]-n, n\left[^{2}\right)^{4}} \sum_{k, m \in\{1,2,3\}^{q}} \sum_{K, M \in\{1,2,3\}^{q}} b_{f}(k, u) b_{f}(m, u) \\
b_{f}(K, u) b_{f}(M, u) \Gamma_{k_{1}, K_{1}}^{U}\left(t_{1}-t_{2}\right) \ldots \Gamma_{k_{q-p}, K_{q-p}}^{U}\left(t_{1}-t_{2}\right) \times \\
\Gamma_{m_{1}, M_{1}}^{U}\left(s_{1}-s_{2}\right) \cdots \Gamma_{m_{q-p}, M_{q-p}}^{U}\left(s_{1}-s_{2}\right) \Gamma_{k_{q-p+1}, m_{q-p+1}}^{U}\left(t_{1}-s_{1}\right) \cdots \times \\
\Gamma_{k_{q}, m_{q}}^{U}\left(t_{1}-s_{1}\right) \Gamma_{K_{q-p+1}, M_{q-p+1}}^{U}\left(t_{2}-s_{2}\right) \cdots \Gamma_{K_{q}, M_{q}}^{U}\left(t_{2}-s_{2}\right) \mathrm{d} t_{1} \mathrm{~d} s_{1} \mathrm{~d} t_{2} \mathrm{~d} s_{2},
\end{gathered}
$$

where $\Gamma^{U}$ has been defined in (4.5).

Using inequality (4.6), we get the bound

$$
\begin{aligned}
& C_{n} \leqslant\left(\frac{1}{2 n}\right)^{4} \mathbf{L}^{2 q}\left(\sum_{m \in\{1,2,3\}^{q}}\left|b_{f}(m, u)\right|\right)^{4} \int_{(]-n, n\left[^{2}\right)^{4}} \Psi^{q-p}\left(A .\left(t_{1}-t_{2}\right)\right) \times \\
& \Psi^{q-p}\left(A .\left(s_{1}-s_{2}\right)\right) \Psi^{p}\left(A \cdot\left(t_{1}-s_{1}\right)\right) \Psi^{p}\left(A .\left(t_{2}-s_{2}\right)\right) \mathrm{d} t_{1} \mathrm{~d} s_{1} \mathrm{~d} t_{2} \mathrm{~d} s_{2} .
\end{aligned}
$$

Moreover, we have

$$
\Psi^{q-p}\left(A .\left(t_{1}-t_{2}\right)\right) \Psi^{p}\left(A .\left(t_{2}-s_{2}\right)\right) \leqslant \Psi^{q}\left(A .\left(t_{1}-t_{2}\right)\right)+\Psi^{q}\left(A .\left(t_{2}-s_{2}\right)\right) .
$$

Furthermore for $r \in \mathbb{N}^{\star}$ and $\left.v \in\right]-n, n\left[{ }^{2}\right.$, one has

$$
\int_{]-n, n\left[^{2}\right.} \Psi^{r}(A \cdot(u-v)) \mathrm{d} u \leqslant \mathbf{C} \int_{\mathbb{R}^{2}} \Psi^{r}(u) \mathrm{d} u<+\infty .
$$

Applying the penultimate and last inequalities to $p \geqslant 1, q \geqslant 1$ and $q-p \geqslant 1$, one obtains

$$
C_{n} \leqslant \mathbf{C}_{\mathbf{q}}\left(\frac{1}{2 n}\right)^{2}\left(\int_{\mathbb{R}^{2}} \Psi^{q}(u) d u\right)\left(\int_{\mathbb{R}^{2}} \Psi^{q-p}(u) \mathrm{d} u\right)\left(\int_{\mathbb{R}^{2}} \Psi^{p}(u) \mathrm{d} u\right),
$$

thus we proved that $\lim _{n \rightarrow+\infty} C_{n}=0$, this achieves proof of Lemma 4.10. 
Hence we proved that,

- for all $Q \geqslant 1, \pi^{Q}\left(\xi_{f}^{(n)}(u)\right) \underset{n \rightarrow+\infty}{\stackrel{L a w}{\longrightarrow}} N\left(0 ; \sum_{q=1}^{Q} V_{q}(u)\right)$, where $V_{q}(u)$ has been defined by (4.7).

On the other hand we proved in Lemma 4.5 that

- for all $n \geqslant 1, \pi^{Q}\left(\xi_{f}^{(n)}(u)\right) \underset{Q \rightarrow+\infty}{\stackrel{L^{2}(\Omega)}{\longrightarrow}} \xi_{f}^{(n)}(u)$, and that

- $\lim _{Q \rightarrow+\infty} \sum_{q=1}^{Q} V_{q}(u)=\Sigma_{f, f}(u)$.

Finally and by Proposition 4.3 we also have

- $\lim _{Q \rightarrow+\infty} \lim _{n \rightarrow+\infty}\left\|\pi^{Q}\left(\xi_{f}^{(n)}(u)\right)-\xi_{f}^{(n)}(u)\right\|_{L^{2}(\Omega)}=0$.

Applying Dynkin [13, Lemma 1.1], we can conclude that $\xi_{f}^{(n)}(u) \underset{n \rightarrow+\infty}{\stackrel{L a w}{\longrightarrow}} N\left(0 ; \Sigma_{f, f}(u)\right)$, that achieves proof of Theorem 4.7.

\section{The affinity estimators}

In this section $\left(T_{n}\right)_{n}$ will be still open bounded squares of $\mathbb{R}^{2}$, with the following form $\left.T_{n}:=\right]-n, n\left[{ }^{2}\right.$ with $n \in \mathbb{N}^{\star}$, and $n$ tends to infinity. Also $u$ will be a fixed level in $\mathbb{R}$.

We propose estimators of the affinity parameters defined as $\lambda$ and $\theta$, by considering the particular functional of the level set $u$ proposed by Wschebor [32, chap. 3.6 F, pages 82-85]. In this aim we reproduce in Sections 5.1, 5.2 and 5.3 all the computations made by Wschebor including the three forthcoming attached pictures. By using the general convergence results established in previous Section 4 we point out convergence properties of these estimators.

\subsection{The functional of interest}

Let $J_{\overrightarrow{f^{\sharp}}}^{(n)}(u)$ this particular functional (see notations given in (4.13) for the definition of a general vector-functional of the level $u$ ), where the vector-function $\overrightarrow{f^{\star}}$ is defined as follows. Let $v^{\star} \in S^{1}$ a fixed vector and consider

$$
\begin{aligned}
& S^{1} \longrightarrow S^{1} \\
& \omega \mapsto \overrightarrow{f^{\star}}(\omega):=\omega \times\left(\mathbb{1}_{\left\{\left\langle\omega, v^{\star}\right\rangle \geqslant 0\right\}}-\mathbb{1}_{\left\{\left\langle\omega, v^{\star}\right\rangle<0\right\}}\right) .
\end{aligned}
$$

Remark 5.1. Note that function $\overrightarrow{f^{\star}}$ is continuous except on the line given by equation $\left\langle\omega, v^{\star}\right\rangle=0$. That is, function $\overrightarrow{f^{\star}}$ is continuous except on points $\left(-v_{2}^{\star}, v_{1}^{\star}\right)$ and $\left(v_{2}^{\star},-v_{1}^{\star}\right)$, where $v_{i}^{\star}, i=1,2$, are the coordinates of the vector $v^{\star}$. Thus $\overrightarrow{f^{\star}}$ belongs to $\mathcal{B}_{2}$.

Remember that in Section 2.2 we have denoted the eigenvalues of $A$ by $\lambda_{1}, \lambda_{2}$, $0<\lambda_{2} \leqslant \lambda_{1}$. Also $0<\lambda \leqslant 1$ has been defined as the quotient of the eigenvalues, $\lambda:=\frac{\lambda_{2}}{\lambda_{1}}$.

Let $P:=\left(v_{1}, v_{2}\right)$ be an orthonormal basis of eigenvectors of matrix $A$, such that $\lambda_{1}$ and $\lambda_{2}$ are their respectives eigenvalues. The vector $v^{\star}$ can always be written in this basis:

$$
v^{\star}=\cos (\theta) v_{1}+\sin (\theta) v_{2} .
$$

It is always possible to choose $-\frac{\pi}{2}<\theta \leqslant \frac{\pi}{2}$.

Indeed, $\theta$ could be the angle between $v^{\star}$ and the eigenvector corresponding to the highest eigenvalue, because of the symmetry with respect to the point $(0,0)$ and the fact that the mapping transforms $\lambda$ into $\frac{1}{\lambda}$ and $\theta$ into $\frac{\pi}{2}-\theta$ has this effect.

By defining $v^{\star \star}:=\cos \left(\theta+\frac{\pi}{2}\right) v_{1}+\sin \left(\theta+\frac{\pi}{2}\right) v_{2}=-\sin (\theta) v_{1}+\cos (\theta) v_{2}$, we thus defined a direct orthonormal basis $\left(v^{\star}, v^{\star \star}\right)$, see Figure 1 . 


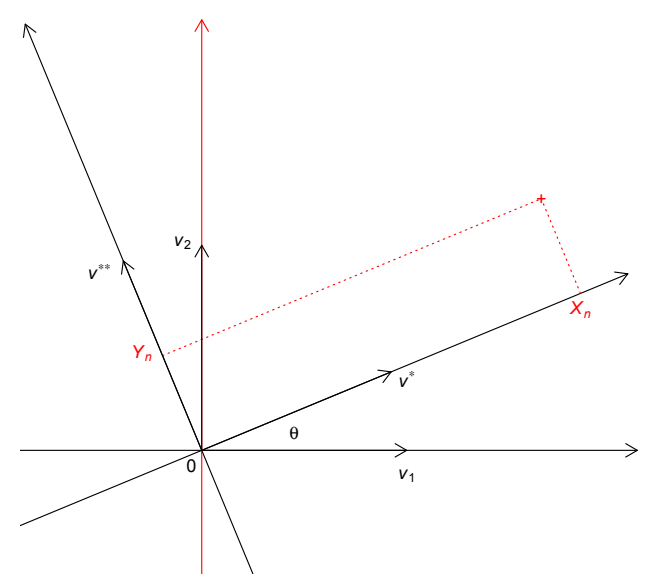

Figure 1: Eigenvector directions

Theorem 4.1 applied to the particular functions $\overrightarrow{f^{\star}}$ and to the function 1 taking values in $\mathbb{R}$ and identically equal to one implies that,

$$
\frac{J_{\overrightarrow{f^{\star}}}^{(n)}(u)}{J_{\mathbf{1}}^{(n)}(u)} \underset{n \rightarrow+\infty}{\stackrel{a . s .}{\longrightarrow}} \frac{\mathbb{E}\left[J_{\overrightarrow{f^{\star}}}^{(1)}(u)\right]}{\mathbb{E}\left[J_{\mathbf{1}}^{(1)}(u)\right]}
$$

Applying the Rice formula for this particular function $\overrightarrow{f^{\star}}$ and for the function 1, we show the following proposition.

\section{Proposition 5.2.}

$$
\frac{J_{\vec{f}^{\star}}^{(n)}(u)}{J_{\mathbf{1}}^{(n)}(u)} \underset{n \rightarrow+\infty}{\stackrel{a . s .}{\longrightarrow}} \frac{1}{I(\lambda)}\left[\left(\cos ^{2}(\theta)+\lambda^{2} \sin ^{2}(\theta)\right)^{\frac{1}{2}} v^{\star}+\frac{\sin (\theta) \cos (\theta)\left(\lambda^{2}-1\right)}{\left(\cos ^{2}(\theta)+\lambda^{2} \sin ^{2}(\theta)\right)^{\frac{1}{2}}} v^{\star \star}\right],
$$

where $I(\lambda)$ is the elliptic integral

$$
I(\lambda):=\int_{0}^{\frac{\pi}{2}}\left(\cos ^{2}(x)+\lambda^{2} \sin ^{2}(x)\right)^{\frac{1}{2}} \mathrm{~d} x .
$$

Remark 5.3. Note that $J_{1}^{(n)}(u)=\frac{\sigma_{1}\left(\mathcal{C}_{n}(u)\right)}{\sigma_{2}\left(T_{n}\right)}$. This functional is nothing but the measurement of the dimensional length of the level set by unit of surface.

Proof of Proposition 5.2. We use a lemma for which a proof is given in Appendix.

\section{Lemma 5.4.}

$$
\frac{\mathbb{E}\left[J_{\vec{f}^{\star}}^{(1)}(u)\right]}{\mathbb{E}\left[J_{1}^{(1)}(u)\right]}=\frac{2}{\pi} \frac{A^{2} \cdot v^{\star}}{\left\|A \cdot v^{\star}\right\|_{2}} \frac{1}{\int_{S^{1}}\|A \cdot \alpha\|_{2} \mathrm{~d} \alpha},
$$

where $\mathrm{d} \alpha$ denotes the normalized area measure on $S^{1}$.

Using last equality we show that $\frac{\mathbb{E}\left[J_{f^{*}}^{\left(\frac{1)}{f^{*}}\right.}(u)\right]}{\mathbb{E}\left[J_{1}^{(1)}(u)\right]}$ can be expressed in the orthonormal basis $\left(v^{\star}, v^{\star \star}\right)$ as follows. One has:

$$
A^{2} \cdot v^{\star}=\lambda_{1}^{2}\left[\cos (\theta) v_{1}+\lambda^{2} \sin (\theta) v_{2}\right],
$$

and

$$
\left\|A \cdot v^{\star}\right\|_{2}=\lambda_{1}\left[\cos ^{2}(\theta)+\lambda^{2} \sin ^{2}(\theta)\right]^{\frac{1}{2}} .
$$


Also

$$
\int_{S^{1}}\|A \cdot \alpha\|_{2} \mathrm{~d} \alpha=\lambda_{1} \int_{0}^{2 \pi}\left[\cos ^{2}(x)+\lambda^{2} \sin ^{2}(x)\right]^{\frac{1}{2}} \frac{\mathrm{d} x}{2 \pi}=\frac{2 \lambda_{1}}{\pi} I(\lambda),
$$

where $I(\lambda)$ is the elliptic integral defined by (5.3).

Thus by using Lemma 5.4 we proved that

$$
\frac{\mathbb{E}\left[J_{f^{\star}}^{(1)}(u)\right]}{\mathbb{E}\left[J_{1}^{(1)}(u)\right]}=\frac{1}{I(\lambda)} \frac{\cos (\theta) v_{1}+\lambda^{2} \sin (\theta) v_{2}}{\left[\cos ^{2}(\theta)+\lambda^{2} \sin ^{2}(\theta)\right]^{\frac{1}{2}}} .
$$

At this stage of the proof we are going to change the basis, that is expressing last identity in the orthonormal basis $\left(v^{\star}, v^{\star \star}\right)$, getting

$$
\frac{\mathbb{E}\left[J_{\overrightarrow{f^{\star}}}^{(1)}(u)\right]}{\mathbb{E}\left[J_{\mathbf{1}}^{(1)}(u)\right]}=\frac{1}{I(\lambda)}\left[\left(\cos ^{2}(\theta)+\lambda^{2} \sin ^{2}(\theta)\right)^{\frac{1}{2}} v^{\star}+\frac{\sin (\theta) \cos (\theta)\left(\lambda^{2}-1\right)}{\left(\cos ^{2}(\theta)+\lambda^{2} \sin ^{2}(\theta)\right)^{\frac{1}{2}}} v^{\star \star}\right] .
$$

Proposition 5.2 ensues from convergence given in (5.2).

We consider function $\vec{F}$ being defined by:

$$
\begin{aligned}
\left.\vec{F}:] 0,1] \times]-\frac{\pi}{2}, \frac{\pi}{2}\right] & \longrightarrow\left\{(X, Y) \in \mathbb{R}^{2}, X>0, X^{2}+Y^{2}<1\right\} \\
(\lambda, \theta) & \mapsto \vec{F}(\lambda, \theta)=\left(F_{1}(\lambda, \theta), F_{2}(\lambda, \theta)\right),
\end{aligned}
$$

where

$$
\begin{cases}F_{1}(\lambda, \theta) & :=\frac{1}{I(\lambda)}\left(\cos ^{2}(\theta)+\lambda^{2} \sin ^{2}(\theta)\right)^{\frac{1}{2}} \\ F_{2}(\lambda, \theta) & :=\frac{1}{I(\lambda)} \frac{\sin (\theta) \cos (\theta)\left(\lambda^{2}-1\right)}{\left(\cos ^{2}(\theta)+\lambda^{2} \sin ^{2}(\theta)\right)^{\frac{1}{2}}}\end{cases}
$$

Proposition 5.2 says that $\frac{J_{f^{\star}}^{(n)}(u)}{J_{1}^{(n)}(u)} \underset{n \rightarrow+\infty}{\stackrel{a . s .}{\longrightarrow}} F_{1}(\lambda, \theta) v^{\star}+F_{2}(\lambda, \theta) v^{\star \star}$.

In order to define estimators for parameters $\lambda$ and $\theta$ we need to look more closely at the properties of function $\vec{F}$. To this end we are going to follow step by step Wschebor's method given in [32, pages 82 to 85 ]. That is the object of next section.

\subsection{The $F$-diffeomorphism}

We prove the following proposition.

Proposition 5.5. Function $\vec{F}$ is a one to one function from $\left.] 0,1[\times]-\frac{\pi}{2}, \frac{\pi}{2}\right]$ onto $\{(X, Y) \in$ $\mathbb{R}^{2}, X>0, X^{2}+Y^{2}<1$ and $\left(X \neq \frac{2}{\pi}\right.$ or $\left.\left.Y \neq 0\right)\right\}$.

Furthermore $\vec{F}$ is a $\mathbf{C}^{2}$-diffeomorphism from the open set $\left.O:=\right] 0,1[\times]-\frac{\pi}{2}, \frac{\pi}{2}[$ onto the open set $O^{\prime}:=\left\{(X, Y) \in \mathbb{R}^{2}, X>0, X^{2}+Y^{2}<1\right.$ and $\left(X>\frac{2}{\pi}\right.$ or $\left.\left.Y \neq 0\right)\right\}$.

Proof of Proposition 5.5. We reproduce here all the computations given by Wschebor in [32] and also attach his two forthcoming pictures.

Let $(X, Y) \in \mathbb{R}^{2}$ be fixed such that $X>0, X^{2}+Y^{2}<1$ and $X \neq \frac{2}{\pi}$ or $Y \neq 0$.

We consider the following system of equations

$$
\left\{\begin{array}{l}
X=F_{1}(\lambda, \theta) \\
Y=F_{2}(\lambda, \theta)
\end{array}\right.
$$

If the system admits a solution $\lambda$, this solution ought to verify the following equation in $\lambda$ :

$$
X^{2} I^{4}(\lambda)\left(X^{2}+Y^{2}\right)-X^{2} I^{2}(\lambda)\left(\lambda^{2}+1\right)+\lambda^{2}=0
$$

At this step, two cases appear: the case where $\left(X^{2}+Y^{2}\right)\left(\frac{\pi}{2}\right)^{2}-1<0$ and the case where $\left(X^{2}+Y^{2}\right)\left(\frac{\pi}{2}\right)^{2}-1 \geqslant 0$. Let us consider the first one. 
1. $\left(X^{2}+Y^{2}\right)\left(\frac{\pi}{2}\right)^{2}-1<0$.

Since for all $x \in[0,1], I(x) \leqslant \frac{\pi}{2}$, the following inequality holds, $\left(X^{2}+Y^{2}\right) I^{2}(\lambda)-1<$ 0 .

Now dividing (5.7) by $I^{2}(\lambda) X^{2}$, one gets

$$
I^{2}(\lambda)\left(X^{2}+Y^{2}\right)-\left(\lambda^{2}+1\right)+\frac{\lambda^{2}}{I^{2}(\lambda) X^{2}}=0,
$$

that is, since $\left(X^{2}+Y^{2}\right) I^{2}(\lambda)-1<0$,

$$
f_{1}(\lambda)=f_{2}(\lambda)
$$

where

$$
\begin{aligned}
& f_{1}(\lambda):=X^{2}\left(\frac{I(\lambda)}{\lambda}\right)^{2} \\
& f_{2}(\lambda):=\frac{X^{2} I^{2}(\lambda)-1}{\left(X^{2}+Y^{2}\right) I^{2}(\lambda)-1} .
\end{aligned}
$$

Equation (5.8) admits a unique solution in the interval $0<\lambda<1$. Let us argue this last assertion.

Since $X>0$, function $f_{1}$ is strictly decreasing, while $f_{2}$ is such that $f_{2}^{\prime}(\lambda)=$ $\frac{2 I(\lambda) I^{\prime}(\lambda)}{\left(\left(X^{2}+Y^{2}\right) I^{2}(\lambda)-1\right)^{2}} Y^{2}>0$, if $Y \neq 0$.

Let us consider the case where $Y \neq 0$. In this case function $f_{2}$ is strictly increasing. By summarizing the situation we know that $f_{1}-f_{2}$ is continuous on $\left.] 0,1\right]$, strictly decreasing and such that $\left(f_{1}-f_{2}\right)\left(0^{+}\right)=+\infty$ and

$\left(f_{1}-f_{2}\right)(1)=\frac{X^{2} Y^{2}\left(\frac{\pi}{2}\right)^{4}+\left(X^{2}\left(\frac{\pi}{2}\right)^{2}-1\right)^{2}}{\left(X^{2}+Y^{2}\right)\left(\frac{\pi}{2}\right)^{2}-1}<0$. Thus there exists a unique $0<\lambda<1$, such that $f_{1}(\lambda)=f_{2}(\lambda)$, see Figure 2 .

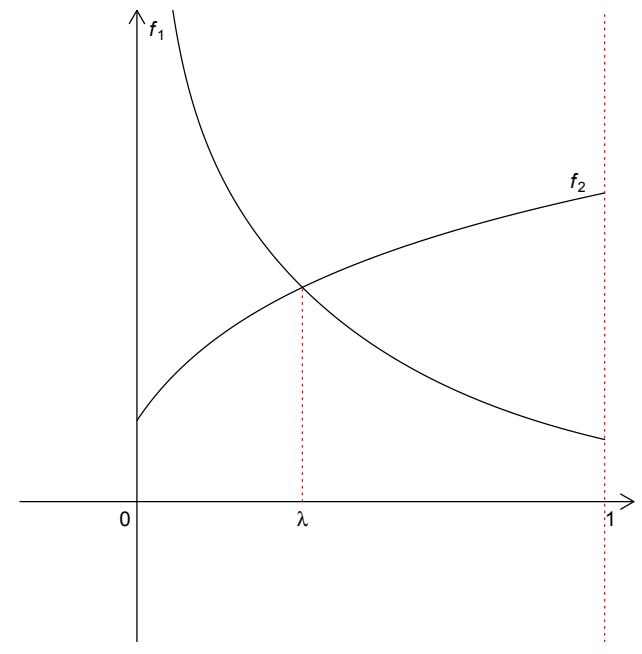

Figure 2: $\lambda$-solution, first case

Now, in the case where $Y:=0, f_{2}(\lambda)=1$. We define $g$ by $g(\lambda):=\frac{I(\lambda)}{\lambda}$. Since $X<\frac{2}{\pi}$, $\lambda=g^{-1}\left(\frac{1}{X}\right)$ is the unique solution in $] 0,1[$ of (5.8).

Let us consider now the second case. 
2. $\left(X^{2}+Y^{2}\right)\left(\frac{\pi}{2}\right)^{2}-1 \geqslant 0$.

Since $X^{2}+Y^{2}>0$, function $h: \lambda \rightarrow\left(X^{2}+Y^{2}\right) I^{2}(\lambda)-1$ is strictly increasing and continuous on $] 0,1]$.

Moreover, $h(0)=X^{2}+Y^{2}-1<0$ and $h(1)=\left(X^{2}+Y^{2}\right)\left(\frac{\pi}{2}\right)^{2}-1 \geqslant 0$. Thus there exists a unique $0<\lambda_{0} \leqslant 1$ such that $h\left(\lambda_{0}\right)=\left(X^{2}+Y^{2}\right) I^{2}\left(\lambda_{0}\right)-1=0$ and then for $\lambda<\lambda_{0}$, one has $\left(X^{2}+Y^{2}\right) I^{2}(\lambda)-1<0$ and for $\lambda>\lambda_{0},\left(X^{2}+Y^{2}\right) I^{2}(\lambda)-1>0$.

Arguing as in first part, we can deduce that if (5.6) admits a solution $\lambda \neq \lambda_{0}$, this solution ought to verify $f_{1}(\lambda)=f_{2}(\lambda)$.

Function $f_{1}$ is strictly decreasing, while function $f_{2}$ is strictly increasing if $Y \neq 0$.

In this case $f_{1}-f_{2}$ is continuous on $\left.] 0,1\right]$, strictly decreasing and such that $\left(f_{1}-\right.$ $\left.f_{2}\right)\left(0^{+}\right)=+\infty$ and $\left(f_{1}-f_{2}\right)\left(\lambda_{0}^{-}\right)=-\infty$, since $Y \neq 0$. Thus there exists a unique $0<\lambda<\lambda_{0} \leqslant 1$, such that $f_{1}(\lambda)=f_{2}(\lambda)$.

On the other side, still if $Y \neq 0$ and if $\lambda_{0}<1$, we have $\left(f_{1}-f_{2}\right)\left(\lambda_{0}^{+}\right)=+\infty$ and $\left(f_{1}-f_{2}\right)\left(1^{-}\right)>0$. Then there is no more solution to (5.8) into interval $\left.] \lambda_{0}^{+}, 1\right]$.

Thus we have proved that in the case where $Y \neq 0$, if (5.6) admits a solution $\lambda \neq \lambda_{0}$, this solution is the unique solution in ]0,1[ of (5.8) and this solution belongs to the interval $] 0, \lambda_{0}[$ see Figure 3.

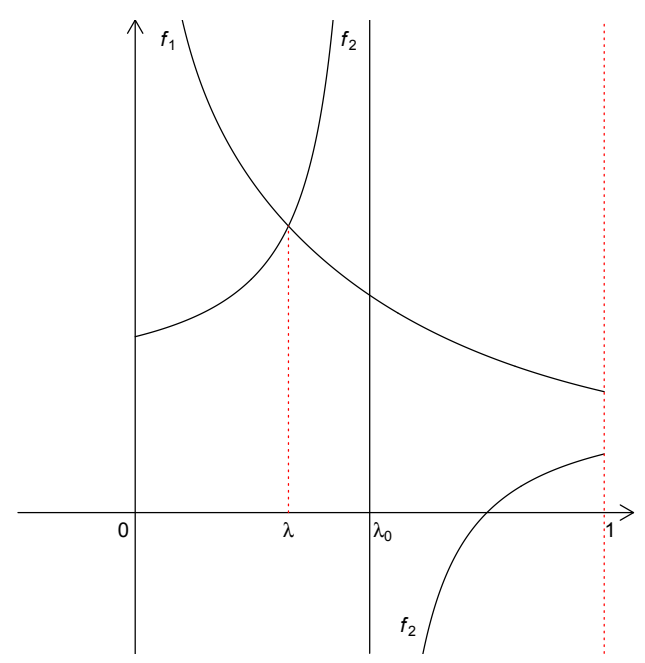

Figure 3: $\lambda$-solution, second case

Now we are going to prove that in the case where $Y=0$, there is no solution to (5.6) different from $\lambda_{0}$.

In fact, if $\lambda$ is such a solution, that is different from $\lambda_{0}$, then $f_{1}(\lambda)=X^{2}\left(\frac{I(\lambda)}{\lambda}\right)^{2}=$ $f_{2}(\lambda)=1$, that is no possible. Indeed, since $X^{2}\left(\frac{\pi}{2}\right)^{2}-1>0$ then we would have $1<X^{2}\left(\frac{\pi}{2}\right)^{2} \leqslant X^{2}\left(\frac{I(\lambda)}{\lambda}\right)^{2}=1$.

To finish the proof of this part of proposition, we look for conditions on $\lambda=\lambda_{0}$ to be solution of (5.6). If such a solution exists necessarily it has to verify (5.7), and then since $\left(X^{2}+Y^{2}\right) I^{2}\left(\lambda_{0}\right)-1=0$, we would have $\lambda_{0}^{2}\left(1-X^{2} I^{2}\left(\lambda_{0}\right)\right)=0$, so that $Y=0$ and $\lambda_{0}=I^{-1}\left(\frac{1}{X}\right)$.

Thus, since $X^{2}\left(\frac{\pi}{2}\right)^{2}>1$, then $0<\lambda=\lambda_{0}=I^{-1}\left(\frac{1}{X}\right)<1$. 
If we combine all results we get the following: if (5.6) admits a solution $\lambda$ then, this solution is unique, satisfies $0<\lambda<1$ and is such that:

- Or $\left(X^{2}+Y^{2}\right)\left(\frac{\pi}{2}\right)^{2}-1<0$, and this solution verifies equation $f_{1}(\lambda)=f_{2}(\lambda)$. Furthermore in the case where $Y=0$ then $\lambda=g^{-1}\left(\frac{1}{X}\right)$, where we recall that

- $\operatorname{gr}(\lambda)=\frac{I(\lambda)}{\operatorname{Or}}\left(X^{2}+Y^{2}\right)\left(\frac{\pi}{2}\right)^{2}-1 \geqslant 0$ and $Y \neq 0$, and this solution verifies equation

- $f_{1}(\lambda)=f_{2}(\lambda)$.

Remark 5.6. Note that in all cases $\lambda$ verifies (5.7).

Remark 5.7. Note that in all cases $\left(X^{2}+Y^{2}\right) I^{2}(\lambda)-1 \leqslant 0$.

Now by continuing the reasoning by necessary condition, if we want that $\lambda<1$ verifies the first equation of (5.6), we have to resolve the following equation:

$$
X^{2} I^{2}(\lambda)=\left(\lambda^{2}-1\right) \sin ^{2}(\theta)+1,
$$

that is

$$
\sin ^{2}(\theta)=\frac{1-X^{2} I^{2}(\lambda)}{1-\lambda^{2}}=\frac{\frac{1}{\lambda^{2}}-f_{1}(\lambda)}{\frac{1}{\lambda^{2}}-1} .
$$

Let us see that $0 \leqslant \frac{\frac{1}{\lambda^{2}}-f_{1}(\lambda)}{\frac{1}{\lambda^{2}}-1} \leqslant 1$. We have the following equivalence:

$$
\left(\frac{\frac{1}{\lambda^{2}}-f_{1}(\lambda)}{\frac{1}{\lambda^{2}}-1} \leqslant 1\right) \Longleftrightarrow\left(f_{1}(\lambda) \geqslant 1\right) .
$$

In the case where $X^{2}\left(\frac{\pi}{2}\right)^{2}>1$ and $Y=0$, we have $\lambda=I^{-1}\left(\frac{1}{X}\right)$. Thus $f_{1}(\lambda)=\frac{1}{\lambda^{2}} \geqslant 1$, since $0<\lambda<1$.

In the other cases, $f_{1}(\lambda)=f_{2}(\lambda)$ and since $f_{2}$ is an increasing function, $f_{1}(\lambda) \geqslant f_{2}\left(0^{+}\right)=$ $\frac{X^{2}-1}{X^{2}+Y^{2}-1} \geqslant 1$, since $Y^{2} \geqslant 0$ and $X^{2}+Y^{2}-1<0$.

Now $\left(\frac{\frac{1}{\lambda^{2}}-f_{1}(\lambda)}{\frac{1}{\lambda^{2}}-1} \geqslant 0\right) \Longleftrightarrow\left(X^{2} I^{2}(\lambda)-1 \leqslant 0\right)$.

Remark 5.7 gives the result.

If we look at the sign of expression $F_{2}(\lambda, \theta)$, we have to set:

$$
\theta:=\arcsin \left(\sqrt{\frac{1-X^{2} I^{2}(\lambda)}{1-\lambda^{2}}}\right)\left(\mathbb{1}_{\{Y \leqslant 0\}}-\mathbb{1}_{\{Y>0\}}\right) .
$$

In this way, we always have $-\frac{\pi}{2} \leqslant \theta \leqslant \frac{\pi}{2}$ and in fact $\theta>-\frac{\pi}{2}$. To verify last inequality, remark that

$$
\begin{array}{r}
\left(\theta=-\frac{\pi}{2}\right) \Longleftrightarrow\left(Y>0 \text { and } X^{2} I^{2}(\lambda)=\lambda^{2}\right) \\
\Longrightarrow\left(Y>0 \text { and } f_{1}(\lambda)=f_{2}(\lambda)=1\right) \Longrightarrow(Y>0 \text { and } Y=0),
\end{array}
$$

which is impossible.

Let us see now that we can go backward. That is let us verify that these proposed $\lambda(<1)$ and $\theta$ are actually solutions of (5.6).

$$
\begin{array}{r}
\left(\sin ^{2}(\theta)=\frac{1-X^{2} I^{2}(\lambda)}{1-\lambda^{2}}\right) \Longleftrightarrow\left(X^{2}=\frac{\cos ^{2}(\theta)+\lambda^{2} \sin ^{2}(\theta)}{I^{2}(\lambda)}\right) \\
\Longleftrightarrow\left(X=\frac{\left(\cos ^{2}(\theta)+\lambda^{2} \sin ^{2}(\theta)\right)^{\frac{1}{2}}}{I(\lambda)}=F_{1}(\lambda, \theta)\right)
\end{array}
$$


last equivalence provides from the fact that $X>0$.

Let us consider the second equation of (5.6). In the case where $Y=0$ and $X>\frac{2}{\pi}$, one has $\lambda=I^{-1}\left(\frac{1}{X}\right)$ so that $\theta=\arcsin (0)=0$ and $Y=0=F_{2}(\lambda, 0)$. In the other cases, one has $f_{1}(\lambda)=f_{2}(\lambda)$, and since $X^{2} I^{2}(\lambda)=\cos ^{2}(\theta)+\lambda^{2} \sin ^{2}(\theta)$ one has

$$
\begin{aligned}
& \left(f_{1}(\lambda)=f_{2}(\lambda)\right) \\
& \Longleftrightarrow\left(\frac{X^{2} I^{2}(\lambda)}{\lambda^{2}}=\frac{X^{2} I^{2}(\lambda)-1}{\left(X^{2}+Y^{2}\right) I^{2}(\lambda)-1}\right) \\
& \Longleftrightarrow\left(Y^{2}=\left[\frac{1}{I(\lambda)} \frac{\sin (\theta) \cos (\theta)\left(\lambda^{2}-1\right)}{\left(\cos ^{2}(\theta)+\lambda^{2} \sin ^{2}(\theta)\right)^{\frac{1}{2}}}\right]^{2}\right) \\
& \Longleftrightarrow\left(Y=\frac{1}{I(\lambda)} \frac{\sin (\theta) \cos (\theta)\left(\lambda^{2}-1\right)}{\left(\cos ^{2}(\theta)+\lambda^{2} \sin ^{2}(\theta)\right)^{\frac{1}{2}}}=F_{2}(\lambda, \theta)\right),
\end{aligned}
$$

last equivalence providing from the fact that if $Y \leqslant 0,0 \leqslant \theta \leqslant \frac{\pi}{2}$ and if $Y>0,-\frac{\pi}{2} \leqslant \theta \leqslant 0$. We thus proved that function $\vec{F}$ is a one to one function from $\left.] 0,1[\times]-\frac{\pi}{2}, \frac{\pi}{2}\right]$ onto $\{(X, Y) \in$ $\mathbb{R}^{2}, X>0, X^{2}+Y^{2}<1$ and $\left(X \neq \frac{2}{\pi}\right.$ or $\left.\left.Y \neq 0\right)\right\}$. This yields the first part of proposition.

Remark 5.8. Note that $\left(\vec{F}(\lambda, \theta)=\left(\frac{2}{\pi}, 0\right)\right) \Longleftrightarrow\left(\lambda=1,-\frac{\pi}{2}<\theta \leqslant \frac{\pi}{2}\right)$.

Now the following equivalence

$$
\left(\lambda<1, \theta=\frac{\pi}{2}\right) \Longleftrightarrow\left(Y=0 \text { and } X<\frac{2}{\pi}\right),
$$

leads to the conclusion that $\vec{F}$ is a one to one function from the open set $O:=$ ] $0,1[\times]-\frac{\pi}{2}, \frac{\pi}{2}$ [ onto the open set $O^{\prime}:=\left\{(X, Y) \in \mathbb{R}^{2}, X>0, X^{2}+Y^{2}<1\right.$ and $(X>$ $\frac{2}{\pi}$ or $\left.\left.Y \neq 0\right)\right\}$. The second part of proposition follows from Lemma A.3 proved in Appendix which ensures that the Jacobian of the tranformation $\vec{F}$ is different from zero since $0<\lambda<1$.

We are now listen to give the definitions of the estimators of parameters $\lambda$ and $\theta$.

\subsection{Definition of the affinity parameters $\widehat{\lambda}_{n}$ and $\widehat{\theta}_{n}$}

As in [32, chap. $3.6 \mathrm{~F}]$ we write the observed ratio of functionals $\frac{J_{f^{\star}}^{(n)}(u)}{J_{1}^{(n)}(u)}$ as

$$
\frac{J_{f^{\star}}^{(n)}(u)}{J_{\mathbf{1}}^{(n)}(u)}=X_{n} v^{\star}+Y_{n} v^{\star \star} .
$$

As a first step we use a lemma proved in Appendix A.

Lemma 5.9. The random variables $X_{n}$ and $Y_{n}$ defined in (5.9) are such that for $n \in \mathbb{N}^{\star}$, a.s. $X_{n}>0$ and $X_{n}^{2}+Y_{n}^{2}<1$.

The first part of Proposition 5.5, Lemma 5.9 and Remark 5.8 provide the following estimators $\widehat{\lambda}_{n}$ and $\widehat{\theta}_{n}$ of parameters $\lambda$ and $\theta$ via the following proposition.

Proposition 5.10. Let consider the following system of equations

$$
\left\{\begin{array}{l}
X_{n}=F_{1}(\lambda, \theta) \\
Y_{n}=F_{2}(\lambda, \theta)
\end{array}\right.
$$

where $\vec{F}=\left(F_{1}, F_{2}\right)$ has been defined by (5.5). 
1. In the case where $Y_{n} \neq 0$ or $X_{n} \neq \frac{2}{\pi}$, it admits a unique solution $\left(\widehat{\lambda}_{n}, \widehat{\theta}_{n}\right)$ such that $0<\widehat{\lambda}_{n}<1$ and $-\frac{\pi}{2}<\widehat{\theta}_{n} \leqslant \frac{\pi}{2}$.

- The estimator $0<\widehat{\lambda}_{n}<1$ is solution of

$$
X_{n}^{2}\left(\frac{I(\lambda)}{\lambda}\right)^{2}=\frac{X_{n}^{2} I^{2}(\lambda)-1}{\left(X_{n}^{2}+Y_{n}^{2}\right) I^{2}(\lambda)-1},
$$

- except for $Y_{n}=0$ and $X_{n}>\frac{2}{\pi}$, where $\widehat{\lambda}_{n}:=I^{-1}\left(\frac{1}{X_{n}}\right)$.

- The estimator $\widehat{\theta}_{n}$ is defined as

$$
\widehat{\theta}_{n}:=\arcsin \left(\sqrt{\frac{1-X_{n}^{2} I^{2}\left(\widehat{\lambda}_{n}\right)}{1-\widehat{\lambda}_{n}^{2}}}\right)\left(\mathbb{1}_{\left\{Y_{n} \leqslant 0\right\}}-\mathbb{1}_{\left\{Y_{n}>0\right\}}\right) .
$$

2. In the case where $Y_{n}=0$ and $X_{n}=\frac{2}{\pi}$, it admits a unique solution $\widehat{\lambda}_{n}:=1, \widehat{\theta}_{n}$ being any number belonging to ] $\left.-\frac{\pi}{2}, \frac{\pi}{2}\right]$.

Now we will apply the result of almost sure convergence highlighted in Proposition 5.2 and the fact that function $\vec{F}$ is a diffeomorphism. We will deduce the almost sure convergence of the estimators of affinity parameters. Then we will use the law convergence result established for general functionals in Corollary 4.9 in the aim to establish a law convergence result for $\frac{J_{f^{\star}}^{(n)}(u)}{J_{1}^{(n)}(u)}$. Once this is done we will deduce some law convergence results for the estimators $\widehat{\lambda}_{n}$ and $\widehat{\theta}_{n}$ and also propose some confidence intervals for the parameters $\lambda$ and $\theta$.

\subsection{Convergence for the affinity estimators}

\subsubsection{Consistency for the estimators}

We are ready to state the following results of consistency for the two proposed estimators $\widehat{\lambda}_{n}$ and $\widehat{\theta}_{n}$ of parameters $\lambda$ and $\theta$.

Theorem 5.11. For $0<\lambda<1$ and $-\frac{\pi}{2}<\theta<\frac{\pi}{2}$, one has

$$
\widehat{\lambda}_{n} \underset{n \rightarrow+\infty}{\stackrel{a . s .}{\longrightarrow}} \lambda \text { and } \widehat{\theta}_{n} \underset{n \rightarrow+\infty}{\stackrel{a . s .}{\longrightarrow}} \theta \text {. }
$$

Proof of Theorem 5.11. By the second part of Proposition 5.5 we know that $F$ is a $\mathbf{C}^{2}$ diffeomorphism from the open set $O=] 0,1[\times]-\frac{\pi}{2}, \frac{\pi}{2}\left[\right.$ onto the open set $O^{\prime}=\{(X, Y) \in$ $\mathbb{R}^{2}, X>0, X^{2}+Y^{2}<1,\left(Y \neq 0\right.$ or $\left.\left.X>\frac{2}{\pi}\right)\right\}$.

Furthermore by using the result convergence given for $\frac{J_{f^{\star}}^{(n)}(u)}{J_{1}^{(n)}(u)}$ in Proposition 5.2, decomposition of this last random variable given in (5.9), we obtain the following convergence result: for all $0<\lambda \leqslant 1$ and $-\frac{\pi}{2}<\theta \leqslant \frac{\pi}{2}$,

$$
\left(X_{n}, Y_{n}\right) \underset{n \rightarrow+\infty}{\stackrel{a . s .}{\longrightarrow}}(X, Y):=\vec{F}(\lambda, \theta) .
$$

If we reduce the definition domain of $(\lambda, \theta)$, that is if we suppose that $0<\lambda<1$ and $-\frac{\pi}{2}<\theta<\frac{\pi}{2}$, then $(X, Y)$ belongs to the open set $O^{\prime}$. If $\vec{F}^{-1}$ denotes the inverse function of $\vec{F}$, thus since $\vec{F}^{-1}$ is continuous from $O^{\prime}$ to $O$, one deduces that almost surely $\vec{F}^{-1}\left(X_{n}, Y_{n}\right)=\left(\widehat{\lambda}_{n}, \widehat{\theta}_{n}\right)$ converges to $\vec{F}^{-1}(X, Y)=(\lambda, \theta)$. This yields Theorem 5.11 .

We have all the elements to prove a limit theorem about the distributions of estimators $\widehat{\lambda}_{n}$ and $\widehat{\theta}_{n}$. Consequently, confidence intervals for the estimated parameters $\lambda$ and $\theta$ can be proposed. 


\subsubsection{Convergence in law for the estimators}

First, we apply results of Section 4.2.2

Recalling that $\overrightarrow{f^{\star}}=\left(f_{1}^{\star}, f_{2}^{\star}\right)$ has been defined in (5.1). We define $\overrightarrow{f_{\star}}:=\left(f_{1}^{\star}, f_{2}^{\star}, f_{3}^{\star}\right)$, with $f_{3}^{\star}: \equiv 1$. Remark that function $\overrightarrow{f_{\star}}$ belongs to $\mathcal{B}_{3}$. By Theorem 4.1 and Corollary 4.9 one can show the following proposition.

Proposition 5.12.

$$
2 n\left(\frac{J_{\overrightarrow{f^{\star}}}^{(n)}(u)}{J_{\mathbf{1}}^{(n)}(u)}-\frac{\mathbb{E}\left[J_{\overrightarrow{f^{\star}}}^{(1)}(u)\right]}{\mathbb{E}\left[J_{\mathbf{1}}^{(1)}(u)\right]}\right) \underset{n \rightarrow+\infty}{\stackrel{\operatorname{Law}}{\longrightarrow}} \mathcal{N}\left(0 ; \Sigma^{\star}(u)\right),
$$

where $\Sigma^{\star}(u)=\Sigma^{\star}\left(u, \lambda_{1}, \lambda_{2}, P\right):=B \Sigma_{\overrightarrow{f_{\star}}}(u) B^{t}$ and

$$
B:=\frac{1}{a_{\mathbf{1}}(\mathbf{0}, u)}\left(\begin{array}{ccr}
1 & 0 & -\frac{a_{f_{1}^{\star}}(\mathbf{0}, u)}{a_{\mathbf{1}}(\mathbf{0}, u)} \\
0 & 1 & -\frac{a_{f_{2}^{\star}}(\mathbf{0}, u)}{a_{\mathbf{1}}(\mathbf{0}, u)}
\end{array}\right),
$$

where $\Sigma_{\overrightarrow{f_{\star}}}(u)$ being defined by (4.14).

Remark 5.13. The expression of coefficients $a_{f_{i}^{\star}}(\cdot, u)$ for $i=1,2$ and of $a_{\mathbf{1}}(\cdot, u)$ are respectively given in Lemma A.4 and Lemma A. 6 of Appendix A.

Remark 5.14. The asymptotic variance matrix $\Sigma^{\star}(u)$ is a non-degenerate matrix.

Proof of Proposition 5.12. Let $f: S^{1} \rightarrow \mathbb{R}$ be a function belonging to $\mathcal{B}_{1}$. Since $\mathbb{E}\left[J_{f}^{(n)}(u)\right]$ $=a_{f}(\mathbf{0}, u)$ the following decomposition ensues

$$
\begin{aligned}
2 n\left(\frac{J_{f}^{(n)}(u)}{J_{\mathbf{1}}^{(n)}(u)}-\frac{\mathbb{E}\left[J_{f}^{(n)}(u)\right]}{\mathbb{E}\left[J_{\mathbf{1}}^{(n)}(u)\right]}\right)=\frac{1}{a_{\mathbf{1}}(\mathbf{0}, u)}\left(\xi_{f}^{(n)}(u)-\frac{a_{f}(\mathbf{0}, u)}{a_{\mathbf{1}}(\mathbf{0}, u)} \xi_{\mathbf{1}}^{(n)}(u)\right) \\
+\left(\frac{1}{J_{\mathbf{1}}^{(n)}(u)}-\frac{1}{a_{\mathbf{1}}(\mathbf{0}, u)}\right)\left(\xi_{f}^{(n)}(u)-\frac{a_{f}(\mathbf{0}, u)}{a_{\mathbf{1}}(\mathbf{0}, u)} \xi_{\mathbf{1}}^{(n)}(u)\right) \\
\simeq \frac{1}{a_{\mathbf{1}}(\mathbf{0}, u)}\left(\xi_{f}^{(n)}(u)-\frac{a_{f}(\mathbf{0}, u)}{a_{\mathbf{1}}(\mathbf{0}, u)} \xi_{\mathbf{1}}^{(n)}(u)\right),
\end{aligned}
$$

the last asymptotic law equivalence providing from Theorem 4.1 and from Theorem 4.7 (remember that the asymptotic equivalence symbol $\simeq$ has been defined in Section 2.1). Applying this reasoning again successively to $f:=f_{1}^{\star}$ and $f:=f_{2}^{\star}$, and using Corollary 4.9 by taking $f_{1}:=f_{1}^{\star}, f_{2}:=f_{2}^{\star}$ and $f_{3}: \equiv \mathbf{1}$, we get Proposition 5.12.

Remark 5.15. Note that this proof highlights the fact that the asymptotic behavior of

$$
2 n\left(\frac{J_{\vec{f}}^{(n)}(u)}{J_{\mathbf{1}}^{(n)}(u)}-\frac{\mathbb{E}\left[J_{\overrightarrow{f^{k}}}^{(1)}(u)\right]}{\mathbb{E}\left[J_{\mathbf{1}}^{(1)}(u)\right]}\right)
$$

is the same as that of $\xi_{\vec{f}}^{(n)}(u)$, where $\vec{f}:=\left(f_{1}, f_{2}\right)$, with $f_{i}:=\frac{f_{i}^{\star}}{a_{\mathbf{1}}(\mathbf{0}, u)}-\frac{a_{f_{i}^{\star}}(\mathbf{0}, u)}{a_{\mathbf{1}}^{2}(\mathbf{0}, u)} \mathbf{1}, i=1,2$.

Proof of Remark 5.14. It remains to prove that the matrix $\Sigma^{\star}(u)$ is positive definite. In this aim, for $f_{1}, f_{2}: S^{1} \rightarrow \mathbb{R}$ functions belonging to $\mathcal{B}_{1}$, let us note $\vec{f}:=\left(f_{1}, f_{2}\right)$ and for $q \in \mathbb{N}^{\star}$,

$$
\left(\Sigma_{f_{1}, f_{2}}(u)\right)_{q}:=\sum_{\substack{\boldsymbol{k}, \boldsymbol{m} \in \mathbb{N}^{3} \\|\boldsymbol{k}|=|\boldsymbol{m}|=q}} a_{f_{1}}(\boldsymbol{k}, u) a_{f_{2}}(\boldsymbol{m}, u) R(\boldsymbol{k}, \boldsymbol{m})
$$


and

$$
\left(\Sigma_{\vec{f}}(u)\right)_{q}:=\left(\left(\Sigma_{f_{i}, f_{j}}(u)\right)_{q}\right)_{1 \leqslant i, j \leqslant 2} .
$$

The proof of Lemma 5.16 is given in Appendix A.

Lemma 5.16. For all $f_{1}, f_{2}: S^{1} \rightarrow \mathbb{R}$ functions belonging to $\mathcal{B}_{1}$ and for all $q \in \mathbb{N}^{\star}$, one has

$$
\operatorname{det}\left(\Sigma_{\vec{f}}(u)\right) \geqslant \sum_{q=1}^{+\infty} \operatorname{det}\left(\Sigma_{\vec{f}}(u)\right)_{q} \geqslant \operatorname{det}\left(\Sigma_{\vec{f}}(u)\right)_{q} .
$$

Thus applying this lemma to functions $f_{i}$ previously defined in Remark 5.15 by $f_{i}:=\frac{f_{i}^{\star}}{a_{\mathbf{1}}(\mathbf{0}, u)}-\frac{a_{f_{i}^{\star}}(\mathbf{0}, u)}{a_{\mathbf{1}}^{2}(\mathbf{0}, u)} \mathbf{1}$, for $i=1,2$, we obtain

$$
\operatorname{det}\left(\Sigma^{\star}(u)\right) \geqslant \operatorname{det}(\Sigma \vec{f}(u))_{2} .
$$

Note that $\operatorname{det}\left(\Sigma_{\vec{f}}(u)\right)_{1}=0$ and this is why we let $q=2$ in Lemma 5.16. Using arguments similar to those given in the proof of Remark 4.4, simple calculations give

$$
\operatorname{det}\left(\Sigma^{\star}(u)\right) \geqslant 4 \times(2 \pi)^{2} \int_{\mathbb{R}^{4}} f_{x}^{2}(s) f_{x}^{2}(t)\left\{g^{2}(s, t)-g(s, t) g(t, s)\right\} \mathrm{d} s \mathrm{~d} t \geqslant 0,
$$

where

$$
g(s, t):=s_{1}^{2} t_{2}^{2} a+s_{1}^{2} t_{1} t_{2} b-s_{1} s_{2} t_{2}^{2} c
$$

with

$$
\begin{aligned}
a & :=\left[\left(d_{11} d_{22}+d_{12} d_{21}\right) A+d_{11} d_{12} B-d_{21} d_{22} C\right] \operatorname{det}(D) \\
b & :=\left[2 d_{11} d_{21} A+d_{11}^{2} B-d_{21}^{2} C\right] \operatorname{det}(D) \\
c & :=\left[-2 d_{12} d_{22} A-d_{12}^{2} B+d_{22}^{2} C\right] \operatorname{det}(D)
\end{aligned}
$$

while

$$
\begin{aligned}
& A:=a_{f_{1}}((2,0,0), u) a_{f_{2}}((0,2,0), u)-a_{f_{1}}((0,2,0), u) a_{f_{2}}((2,0,0), u) \\
& B:=a_{f_{1}}((2,0,0), u) a_{f_{2}}((1,1,0), u)-a_{f_{1}}((1,1,0), u) a_{f_{2}}((2,0,0), u) \\
& C:=a_{f_{1}}((0,2,0), u) a_{f_{2}}((1,1,0), u)-a_{f_{1}}((1,1,0), u) a_{f_{2}}((0,2,0), u),
\end{aligned}
$$

and $D$ is defined as in the end of the proof of Remark 4.4 as $D=\left(d_{i j}\right)_{1 \leqslant i, j \leqslant 2}:=\frac{1}{\sqrt{\mu}} \Lambda^{-1} P^{t}$. The positivity of the last integral provides from an application of Hölder inequality.

Let us remark on the one hand that the nullity of the integral is equivalent to the equality in Hölder inequality and thus to $a=b=c=0$. On the other hand since $\operatorname{det}(D) \neq 0$, $A, B, C$ is solution of a Cramer linear system. We deduce that $\Sigma^{\star}(u)$ will be strictly positive if $(A, B, C) \neq(0,0,0)$.

Let us see that $C \neq 0$.

By using Lemmas A.4 and A.6, straightforward computations show that,

$$
C=-\frac{\operatorname{det}(P)}{a_{1}^{2}(0,0)} \times \frac{\lambda_{1} \lambda_{2} \mu}{\pi\left(\lambda_{1}^{2}\left(\omega_{1}^{\star}\right)^{2}+\lambda_{2}^{2}\left(\omega_{2}^{\star}\right)^{2}\right)} \times\left[\lambda_{2}^{2}\left(\omega_{2}^{\star}\right)^{2}+2\left(\lambda_{1}^{2}\left(\omega_{1}^{\star}\right)^{2}-\lambda_{2}^{2}\left(\omega_{2}^{\star}\right)^{2}\right) W\right],
$$

where

$$
W:=\frac{\sum_{n=0}^{+\infty} \frac{1}{4(n+1)} V_{n}}{\sum_{n=0}^{+\infty} V_{n}}, \text { while for } n \in \mathbb{N}, V_{n}:=\frac{(2 n) !(2 n+1) !}{(n !)^{4} 2^{4 n+1}}\left(1-\lambda^{2}\right)^{n} .
$$

By using that $0<W \leqslant \frac{1}{4}$, one easily gets that $\lambda_{2}^{2}\left(\omega_{2}^{\star}\right)^{2}+2\left(\lambda_{1}^{2}\left(\omega_{1}^{\star}\right)^{2}-\lambda_{2}^{2}\left(\omega_{2}^{\star}\right)^{2}\right) W>0$, thus $C \neq 0$.

This yields proof of remark. 
We translate the convergence result expressed in the above Proposition 5.12 in the basis $\left(v^{\star}, v^{\star \star}\right)$, recalling that function $\vec{F}$ has been defined in (5.5).

The decomposition given in (5.9), Proposition 5.10 and equality (5.4) imply that for $0<\lambda \leqslant 1$ and $-\frac{\pi}{2}<\theta \leqslant \frac{\pi}{2}$,

$$
\begin{aligned}
& 2 n\left(\frac{J_{f^{\star}}^{(n)}(u)}{J_{\mathbf{1}}^{(n)}(u)}-\frac{\mathbb{E}\left[J_{\vec{f}^{\star}}^{(n)}(u)\right]}{\mathbb{E}\left[J_{1}^{(n)}(u)\right]}\right)=2 n\left(F_{1}\left(\widehat{\lambda}_{n}, \widehat{\theta}_{n}\right)-F_{1}(\lambda, \theta)\right) v^{\star} \\
& +2 n\left(F_{2}\left(\widehat{\lambda}_{n}, \widehat{\theta}_{n}\right)-F_{2}(\lambda, \theta)\right) v^{\star \star} \underset{n \rightarrow+\infty}{\stackrel{L a w}{\longrightarrow}} \mathcal{N}\left(0 ; \Sigma^{\star}(u)\right) .
\end{aligned}
$$

Thus we get for $0<\lambda \leqslant 1$ and $-\frac{\pi}{2}<\theta \leqslant \frac{\pi}{2}$

$$
2 n\left(\vec{F}\left(\widehat{\lambda}_{n}, \widehat{\theta}_{n}\right)-\vec{F}(\lambda, \theta)\right) \underset{n \rightarrow+\infty}{\stackrel{L a w}{\longrightarrow}} \mathcal{N}\left(0 ; \Sigma_{Q}^{\star}(u)\right),
$$

where $\Sigma_{Q}^{\star}(u)=\Sigma_{Q}^{\star}\left(u, \lambda_{1}, \lambda_{2}, P\right):=Q \times \Sigma^{\star}(u) \times Q^{t}$ and $Q$ is the change of basis matrix from the canonical basis $(\vec{i}, \vec{j})$ to the basis $\left(v^{\star}, v^{\star \star}\right)$.

By using last result convergence and the fact that $\vec{F}$ is a $\mathbf{C}^{2}$-diffeomorphism, we get the following theorem.

Theorem 5.17. For $0<\lambda<1$ and $-\frac{\pi}{2}<\theta<\frac{\pi}{2}$, one has

$$
2 n\left(\widehat{\lambda}_{n}-\lambda ; \widehat{\theta}_{n}-\theta\right) \underset{n \rightarrow+\infty}{\stackrel{L a w}{\longrightarrow}} \mathcal{N}\left(0 ; \Sigma_{\lambda, \theta}(u)\right)
$$

where $\Sigma_{\lambda, \theta}(u):=C(\lambda, \theta) \times \Sigma_{Q}^{\star}(u) \times C^{t}(\lambda, \theta)$ and

$$
C(\lambda, \theta):=\frac{1}{J_{\vec{F}}(\lambda, \theta)}\left(\begin{array}{cc}
\frac{\partial F_{2}}{\partial \theta}(\lambda, \theta) & -\frac{\partial F_{1}}{\partial \theta}(\lambda, \theta) \\
-\frac{\partial F_{2}}{\partial \lambda}(\lambda, \theta) & \frac{\partial F_{1}}{\partial \lambda}(\lambda, \theta)
\end{array}\right)
$$

where the Jacobian $J_{\vec{F}}$ has been defined in Appendix (A.2).

Proof of Theorem 5.17. We supposed that $(\lambda, \theta) \in O=] 0,1[\times]-\frac{\pi}{2}, \frac{\pi}{2}[$. On the one hand, by Proposition 5.5, we know that $\vec{F}$ is a $\mathbf{C}^{2}$-diffeomorphism from the open set $O$ onto the open set $O^{\prime}=\vec{F}(O)$.

On the other hand, by Theorem 5.11, we know that

$$
\left(\widehat{\lambda}_{n}, \widehat{\theta}_{n}\right) \underset{n \rightarrow+\infty}{\stackrel{a . s .}{\longrightarrow}}(\lambda, \theta) \text {. }
$$

Thus for $n$ large enough, almost surely $\vec{F}\left(\widehat{\lambda}_{n}, \widehat{\theta}_{n}\right)$ and $\vec{F}(\lambda, \theta)$ belong to $O^{\prime}$. Using a second order Taylor-Young expansion of $\vec{F}^{-1}$ about $\vec{F}(\lambda, \theta)$, we get

$$
\begin{aligned}
& 2 n\left(\widehat{\lambda}_{n}-\lambda ; \widehat{\theta}_{n}-\theta\right)= \\
& \qquad \sum_{j=1}^{2} \frac{\partial \vec{F}^{-1}}{\partial x_{j}}(\vec{F}(\lambda, \theta)) \times 2 n\left(F_{j}\left(\widehat{\lambda}_{n}, \widehat{\theta}_{n}\right)-F_{j}(\lambda, \theta)\right)+ \\
& \quad \frac{1}{2} \sum_{j, k=1}^{2} \frac{\partial^{2} \vec{F}^{-1}}{\partial x_{j} \partial x_{k}}(\vec{F}(\lambda, \theta)) \times 2 n\left(F_{j}\left(\widehat{\lambda}_{n}, \widehat{\theta}_{n}\right)-\right. \\
& \left.F_{j}(\lambda, \theta)\right) \times\left(F_{k}\left(\widehat{\lambda}_{n}, \widehat{\theta}_{n}\right)-F_{k}(\lambda, \theta)\right) \\
& \quad+o\left(2 n\left\|\vec{F}\left(\widehat{\lambda}_{n}, \widehat{\theta}_{n}\right)-\vec{F}(\lambda, \theta)\right\|_{2}^{2}\right) .
\end{aligned}
$$

Law convergence result expressed in (5.11) gives Theorem 5.17. 
Now we are in position to propose confidence intervals for $(\lambda, \theta)$ when the covariance $r_{z}$ of $Z$ is known.

\subsubsection{Confidence intervals for the affinity parameters}

In this section, we suppose that parameters $\lambda$ and $\theta$ are such that $0<\lambda<1,-\frac{\pi}{2}<\theta<\frac{\pi}{2}$. We will also assume that the covariance function $r_{z}$ is a known function.

One can build confidence intervals for parameters $(\lambda, \theta)$. We will show that

$$
\Sigma^{\star}(u)=\Sigma^{\star}\left(u, \lambda_{1}, \lambda_{2}, P\right)=\frac{1}{\lambda_{1}^{2}} \Sigma_{\star}(u, \lambda, P),
$$

where $\Sigma_{\star}$ is a continuous matrix as function of $(\lambda, P)$ and is computable provided that $(\lambda, P)$ are given.

We then consider $\widehat{\lambda}_{1, n}$ and $\widehat{P}_{n}$ two estimators of respectively $\lambda_{1}$ and matrix $P$ obtained as follows. We propose $\widehat{P}_{n}:=\left(\widehat{v}_{1, n}, \widehat{v}_{2, n}\right)$ as estimator of $P=\left(v_{1}, v_{2}\right)$, the orthonormal basis of eigenvectors of matrix $A$, with:

$$
\left\{\begin{array}{l}
\widehat{v}_{1, n}:=\cos \left(\widehat{\theta}_{n}\right) v^{\star}-\sin \left(\widehat{\theta}_{n}\right) v^{\star \star} \\
\widehat{v}_{2, n}:=\sin \left(\widehat{\theta}_{n}\right) v^{\star}+\cos \left(\widehat{\theta}_{n}\right) v^{\star \star}
\end{array}\right.
$$

By Theorem 5.11, $\widehat{P}_{n}$ is a consistent estimator of $P$.

Now for $\widehat{\lambda}_{1, n}$, first, we apply Theorem 4.1 to the particular function $f \equiv \mathbf{1}$ and then, we use the result of Proposition 3.10.

We deduce that if $\widetilde{\Lambda}(\lambda):=\left(\begin{array}{ll}1 & 0 \\ 0 & \lambda\end{array}\right)$, one has

$$
J_{1}^{(n)}(u) \underset{n \rightarrow+\infty}{\stackrel{a . s .}{\longrightarrow}} \lambda_{1} p_{Z(0)}(u) \mathbb{E}\left[\left\|\widetilde{\Lambda}(\lambda) P^{t} . \nabla Z(0)\right\|_{2}\right]=\lambda_{1} \Phi(u, \lambda, P),
$$

where $\Phi$ is a continuous function of its arguments.

Once again by applying Theorem 5.11 one gets a consistent estimator for $\lambda_{1}$ by taking:

$$
\widehat{\lambda}_{1, n}:=\frac{J_{1}^{(n)}(u)}{\Phi\left(u, \widehat{\lambda}_{n}, \widehat{P}_{n}\right)},
$$

and finally a consistent estimator of $\Sigma^{\star}(u)$ is given by

$$
\widehat{\Sigma_{n}^{\star}}(u):=\frac{1}{\widehat{\lambda}_{1, n}^{2}} \Sigma_{\star}\left(u, \widehat{\lambda}_{n}, \widehat{P}_{n}\right) .
$$

The matrix $\widehat{\Sigma_{n}^{\star}}(u)$ is computable and can be factorized as: $\widehat{\Sigma_{n}^{\star}}(u)=R_{n} \Gamma_{n}^{\star} R_{n}^{t}$, where $R_{n}$ is an unitary matrix and $\Gamma_{n}^{\star}$ is a diagonal matrix.

Remark that $\Gamma_{n}^{\star}$ is invertible since $\Sigma^{\star}(u)$ is non-degenerate (see Remark 5.14). Thus let:

$$
D_{n}(u):=\left(C^{-1}\left(\widehat{\lambda}_{n}, \widehat{\theta}_{n}\right)\right)^{t} Q R_{n}\left(\Gamma_{n}^{\star}\right)^{-\frac{1}{2}} R_{n}^{t}
$$

Theorem 5.17 implies Corollary 5.18.

Corollary 5.18. For $0<\lambda<1$ and $-\frac{\pi}{2}<\theta<\frac{\pi}{2}$, one has

$$
2 n\left(\widehat{\lambda}_{n}-\lambda ; \widehat{\theta}_{n}-\theta\right) \cdot D_{n}(u) \underset{n \rightarrow+\infty}{\stackrel{L a w}{\longrightarrow}} \mathcal{N}\left(0 ; I_{2}\right) \text {. }
$$


Proof of Corollary 5.18. First, let us prove that $\Sigma^{\star}(u)=\Sigma^{\star}\left(u, \lambda_{1}, \lambda_{2}, P\right)=\frac{1}{\lambda_{1}^{2}} \Sigma_{\star}(u, \lambda, P)$, where $\Sigma_{\star}$ is a computable and a continuous matrix as function of $(\lambda, P)$.

On the one hand, this fact provides from the form of the ratio of coefficients $a_{f_{i}^{*}}(\boldsymbol{k}, u) / a_{\mathbf{1}}(\mathbf{0}, u), i=1,2,3$, these latter coefficients being defined in Lemmas A.4 and A.6 of Appendix A. Indeed these ratios only depend on the ratio $\lambda=\lambda_{2} / \lambda_{1}$ and $P$ and they are computable as function of $\lambda$ and $P$. They do not depend on $\mu$. On the other hand by Lemma 3.9 given in Section 3.2 one can see that the term $R(\boldsymbol{k}, \boldsymbol{m})$ only depends on the covariance function of $U$ through the following form. By defining $W(v)$ as the 3-dimensional vector $W(v):=\left(\frac{P^{t}}{\sqrt{\mu}} \cdot \nabla Z(v), \frac{Z(v)}{\sqrt{r_{z}(0)}}\right)^{t}$, one has

$$
\begin{aligned}
R(\boldsymbol{k}, \boldsymbol{m})=\int_{\mathbb{R}^{2}} \mathbb{E}\left[\widetilde{H}_{\boldsymbol{k}}(U(0))\right. & \left.\widetilde{H}_{\boldsymbol{m}}(U(v))\right] \mathrm{d} v \\
& =\frac{1}{\lambda_{1}^{2} \lambda} \int_{\mathbb{R}^{2}} \mathbb{E}\left[\widetilde{H}_{\boldsymbol{k}}(W(0)) \widetilde{H}_{\boldsymbol{m}}(W(v))\right] \mathrm{d} v=\frac{1}{\lambda_{1}^{2}} G(\lambda, P, \boldsymbol{k}, \boldsymbol{m}),
\end{aligned}
$$

and since $r_{z}$ is supposed to be known, $G$ is computable as function of $\lambda$ and $P$. Thus $\Sigma^{\star}(u)=\frac{1}{\lambda_{1}^{2}} \Sigma_{\star}(u, \lambda, P)$, and $\Sigma_{\star}$ is computable.

It remains to prove that $\Sigma_{\star}$ is a continuous matrix as function of $\lambda$ and $P$. In this aim, let us compute $\Sigma_{f_{1}, f_{1}}(u)$, similar arguments would be raised for $\Sigma_{f_{2}, f_{2}}(u)$ and for $\Sigma_{f_{1}, f_{2}}(u)$, where we recall that $f_{i}=\frac{f_{i}^{\star}}{a_{1}(\mathbf{0}, u)}-\frac{a_{f_{i}^{\star}}(\mathbf{0}, u)}{a_{\mathbf{1}}^{2}(\mathbf{0}, u)} \mathbf{1}, i=1,2$ (see Remark 5.15).

Applying Proposition 4.3 to $f:=f_{1}$, the one order Rice formula for $\mathbb{E}\left[J_{f_{1}}^{(n)}(u)\right]$ (see Proposition 3.10) and the second order Rice formula for $\mathbb{E}\left[J_{f_{1}}^{(n)}(u)\right]^{2}$ (see second part of Lemma 3.2), it is easy to see that another expression for $\Sigma_{f_{1}, f_{1}}(u)$ is

$$
\begin{aligned}
\Sigma_{f_{1}, f_{1}}(u)=\int_{\mathbb{R}^{2}}\left\{\mathbb { E } \left[f_{1}\left(\nu_{X}(t)\right)\right.\right. & \left.f_{1}\left(\nu_{X}(0)\right)\|\nabla X(t)\|_{2}\|\nabla X(0)\|_{2} \mid X(0)=X(t)=u\right] \\
& \left.\times p_{X(0), X(t)}(u, u)-\left(\mathbb{E}\left[f_{1}\left(\nu_{X}(0)\right)\|\nabla X(0)\|_{2}\right]\right)^{2} p_{X(0)}^{2}(u)\right\} \mathrm{d} t .
\end{aligned}
$$

Denoting by $B(\lambda, P):=P \widetilde{\Lambda}(\lambda) P^{t}$ and letting $v:=A$.t, one gets

$$
\begin{array}{r}
\Sigma_{f_{1}, f_{1}}(u)=\frac{1}{a_{\mathbf{1}}^{2}(\mathbf{0}, u)} \frac{1}{\lambda} \int_{\mathbb{R}^{2}}\left\{E \left[g_{1}\left(\frac{B(\lambda, P) \cdot \nabla Z(v)}{\|B(\lambda, P) \cdot \nabla Z(v)\|_{2}}\right) g_{1}\left(\frac{B(\lambda, P) \cdot \nabla Z(0)}{\|B(\lambda, P) \cdot \nabla Z(0)\|_{2}}\right)\right.\right. \\
\left.\|B(\lambda, P) \cdot \nabla Z(v)\|_{2}\|B(\lambda, P) \cdot \nabla Z(0)\|_{2} / Z(0)=Z(v)=u\right] p_{Z(0), Z(v)}(u, u) \\
\left.-\left(\mathbb{E}\left[g_{1}\left(\frac{B(\lambda, P) \cdot \nabla Z(0)}{\|B(\lambda, P) \cdot \nabla Z(0)\|_{2}}\right)\|B(\lambda, P) \cdot \nabla Z(0)\|_{2}\right]\right)^{2} p_{Z(0)}^{2}(u)\right\} \mathrm{d} v
\end{array}
$$

where $g_{1}:=f_{1}^{\star}-\frac{a_{f_{1}^{\star}}(\mathbf{0}, u)}{a_{\mathbf{1}}(\mathbf{0}, u)}:=f_{1}^{\star}-b(u, \lambda, P)$, while $b$ is a continuous function of $\lambda$ and $P$. We conclude the argument noticing that $a_{\mathbf{1}}(\mathbf{0}, u)=\lambda_{1} c(u, \lambda)$ with $c$ a strictly positive continuous function of $\lambda$.

Now to prove Corollary 5.18, we have Lemma 5.19 proved in Appendix A.

Lemma 5.19. Let $\Sigma:=R \Gamma R^{t}$ a definite positive matrix such that $R$ is an unitary matrix, while $\Gamma$ is a diagonal one. Let also $\left(\Sigma_{n}\right)_{n}$ be an approximation of matrix $\Sigma$, i.e. $\lim _{n \rightarrow+\infty} \Sigma_{n}=\Sigma$, such that $\Sigma_{n}:=R_{n} \Gamma_{n} R_{n}^{t}$ with $R_{n}$ an unitary matrix and $\Gamma_{n}$ a diagonal one. Consider $B_{n}$ a square root of $\Sigma_{n}$, that is $B_{n}:=R_{n} \Gamma_{n}^{\frac{1}{2}} R_{n}^{t}$. Then $\lim _{n \rightarrow+\infty} B_{n}=B:=$ $R \Gamma^{\frac{1}{2}} R^{t}$.

Turning back to the proof of Corollary 5.18, we apply Lemma 5.19 to $\Sigma:=\Sigma^{\star}(u)=$ $R \Gamma^{\star} R^{t}, \Sigma_{n}:=\widehat{\Sigma_{n}^{\star}}(u)=R_{n} \Gamma_{n}^{\star} R_{n}^{t}$. Since $\widehat{\Sigma}_{n}^{\star}(u)$ is a consistent estimator of $\Sigma^{\star}(u)$, we 
Estimation of local anisotropy based on level sets

deduce that $\lim _{n \rightarrow+\infty} \Sigma_{n}=\Sigma$. Using Lemma 5.19 and Theorem 5.11, we obtain that almost surely

$$
\lim _{n \rightarrow+\infty} D_{n}(u)=D(u):=\left(C^{-1}(\lambda, \theta)\right)^{t} Q R\left(\Gamma^{\star}\right)^{-\frac{1}{2}} R^{t} .
$$

Using Theorem 5.17 and the fact that $D^{t}(u) \Sigma_{\lambda, \theta}(u) D(u)=I_{2}$, one finally proved corollary.

We deal in previous Section 5.4 with the convergence for parameters $\lambda$ and $\theta$ when they are such that $0<\lambda<1$ and $-\frac{\pi}{2}<\theta<\frac{\pi}{2}$. In next section we will complete these convergence results by focusing on the case where $\lambda=1$ that will lead naturally to an isotropy test.

\section{Towards a test of isotropy}

\subsection{Complementary results for estimating the parameter $\lambda$}

\subsubsection{Almost sure convergence for $\hat{\lambda}_{n}$}

We emphasize that convergence result in Theorem 5.11 is valid under the assumption that $0<\lambda<1$ and $-\frac{\pi}{2}<\theta<\frac{\pi}{2}$. However, we can better elaborate what is happening to $\widehat{\lambda}_{n}$ in the isotropic case, when $\lambda=1$ (and $-\frac{\pi}{2}<\theta \leqslant \frac{\pi}{2}$ ) and also when $0<\lambda<1$ and $\theta=\frac{\pi}{2}$, via the following theorem.

Theorem 6.1. For $\lambda=1$ and $-\frac{\pi}{2}<\theta \leqslant \frac{\pi}{2}$ or for $0<\lambda<1$ and $\theta=\frac{\pi}{2}$, one has

$$
\widehat{\lambda}_{n} \underset{n \rightarrow+\infty}{\stackrel{a . s .}{\longrightarrow}} \lambda \text {. }
$$

Proof of Theorem 6.1. First let us consider the isotropic case, that is the case where $\lambda=1$ and $\theta$ being any parameter belonging to $\left.]-\frac{\pi}{2}, \frac{\pi}{2}\right]$. By Remark 5.6 stated at the end of Proposition 5.5 proof, the estimator $\widehat{\lambda}_{n}$ has to verify the following equation

$$
X_{n}^{2} I^{4}\left(\widehat{\lambda}_{n}\right)\left(X_{n}^{2}+Y_{n}^{2}\right)-X_{n}^{2} I^{2}\left(\widehat{\lambda}_{n}\right)\left(\widehat{\lambda}_{n}^{2}+1\right)+\widehat{\lambda}_{n}^{2}=0
$$

Thus

$$
\begin{aligned}
\left(X_{n}^{2}-\left(\frac{2}{\pi}\right)^{2}\right)[ & \left.I^{4}\left(\widehat{\lambda}_{n}\right)\left(X_{n}^{2}+Y_{n}^{2}\right)+\left(\frac{2}{\pi}\right)^{2} I^{4}\left(\widehat{\lambda}_{n}\right)-I^{2}\left(\widehat{\lambda}_{n}\right)\left(\widehat{\lambda}_{n}^{2}+1\right)\right] \\
& +\left(\frac{2}{\pi}\right)^{2} I^{4}\left(\widehat{\lambda}_{n}\right) Y_{n}^{2}-\left(\frac{2}{\pi}\right)^{4}\left(\left(\frac{\pi}{2}\right)^{2}-I^{2}\left(\widehat{\lambda}_{n}\right)\right)\left(I^{2}\left(\widehat{\lambda}_{n}\right)-\left(\frac{\pi}{2}\right)^{2} \widehat{\lambda}_{n}^{2}\right)=0 .
\end{aligned}
$$

We define the function $h$ for $0<\lambda \leqslant 1$, by

$$
h(\lambda):=\left(\frac{\pi}{2}-I(\lambda)\right)\left(I(\lambda)-\frac{\pi}{2} \lambda\right) .
$$

By using convergence given in (5.10), we establish that

$$
\left(X_{n}, Y_{n}\right) \underset{n \rightarrow+\infty}{\stackrel{a . s .}{\longrightarrow}}\left(\frac{2}{\pi}, 0\right)
$$

and since $0<\widehat{\lambda}_{n} \leqslant 1$ and $1<I\left(\widehat{\lambda}_{n}\right) \leqslant \frac{\pi}{2}$, we finally showed that

$$
h\left(\widehat{\lambda}_{n}\right) \underset{n \rightarrow+\infty}{\stackrel{a . s .}{\longrightarrow}} 0 .
$$

Since $h$ is a strictly decreasing continuous function on $] 0,1]$ and $h(1)=0$, we obtain that

$$
\widehat{\lambda}_{n} \underset{n \rightarrow+\infty}{\stackrel{a . s .}{\longrightarrow}} 1
$$


that is the required convergence.

Let us look now at the case where $0<\lambda<1$ and $\theta=\frac{\pi}{2}$.

Convergence established in (5.10) now gives that

$$
\left(X_{n}, Y_{n}\right) \underset{n \rightarrow+\infty}{\stackrel{a . s .}{\longrightarrow}}(X, Y)=\left(\frac{\lambda}{I(\lambda)}, 0\right) .
$$

In the same way as before and using Remark 5.6, one gets the following equality.

$$
\begin{aligned}
& \left(X_{n}^{2}-X^{2}\right)\left[I^{4}\left(\widehat{\lambda}_{n}\right)\left(X_{n}^{2}+Y_{n}^{2}\right)+X^{2} I^{4}\left(\widehat{\lambda}_{n}\right)-I^{2}\left(\widehat{\lambda}_{n}\right)\left(\widehat{\lambda}_{n}^{2}+1\right)\right]+X^{2} I^{4}\left(\widehat{\lambda}_{n}\right) Y_{n}^{2} \\
& -\left(\frac{1}{I(\lambda)}\right)^{4}\left(I^{2}(\lambda)-\lambda^{2} I^{2}\left(\widehat{\lambda}_{n}\right)\right)\left(\lambda I\left(\widehat{\lambda}_{n}\right)+I(\lambda) \widehat{\lambda}_{n}\right)\left(\lambda I\left(\widehat{\lambda}_{n}\right)-I(\lambda) \widehat{\lambda}_{n}\right)=0
\end{aligned}
$$

For fixed $0<\lambda<1$, let us define function $f$ by

$$
f(x):=\lambda I(x)-I(\lambda) x, \text { for } 0 \leqslant x \leqslant 1 .
$$

Previous almost sure convergence and the fact that $0<\widehat{\lambda}_{n} \leqslant 1,1<I\left(\widehat{\lambda}_{n}\right) \leqslant \frac{\pi}{2}$ and for $\lambda<1, I(\lambda)-\lambda I\left(\widehat{\lambda}_{n}\right)>I(\lambda)-\frac{\pi}{2}>0$, imply that

$$
f\left(\widehat{\lambda}_{n}\right) \underset{n \rightarrow+\infty}{\stackrel{a . s .}{\longrightarrow}} 0 .
$$

A straightforward calculation shows that function $f$ is a strictly decreasing continuous function on $[0,1]$ such that $f(\lambda)=0$. We deduce that

$$
\widehat{\lambda}_{n} \underset{n \rightarrow+\infty}{\stackrel{a . s .}{\longrightarrow}} \lambda
$$

that yields Theorem 6.1.

We thus set up a first approach to detect if the process $X$ is isotropic or not.

\subsubsection{Convergence in law for $\widehat{\lambda}_{n}$}

In case where $0<\lambda<1$ and $-\frac{\pi}{2}<\theta<\frac{\pi}{2}$, Theorem 5.17 gives as corollary a convergence law result for $2 n\left(\widehat{\lambda}_{n}-\lambda\right)$. Here we complete the statement of this convergence under the assumption that $\lambda=1$ (and $-\frac{\pi}{2}<\theta \leqslant \frac{\pi}{2}$ ) or when $0<\lambda<1$ and $\theta=\frac{\pi}{2}$ via the following theorem.

Theorem 6.2. 1. For $0<\lambda<1$ and $\theta=\frac{\pi}{2}$, one has

$$
2 n\left(\widehat{\lambda}_{n}-\lambda\right) \underset{n \rightarrow+\infty}{\stackrel{L a w}{\longrightarrow}} \mathcal{N}\left(0 ;\left(\Sigma_{\lambda, \frac{\pi}{2}}(u)\right)_{11}\right)
$$

where $\left(\Sigma_{\lambda, \frac{\pi}{2}}(u)\right)_{11}$ stands for the first row and first column element located in matrix $\Sigma_{\lambda, \frac{\pi}{2}}(u)$ defined in Theorem 5.17 .

2. For $\lambda=1$ and $-\frac{\pi}{2}<\theta \leqslant \frac{\pi}{2}$, one has

$$
2 n\left(1-\widehat{\lambda}_{n}\right) \underset{n \rightarrow+\infty}{\stackrel{L a w}{\longrightarrow}} \sqrt{V},
$$

where the density $f_{V}(t)$ of the positive random variable $V$ is given by:

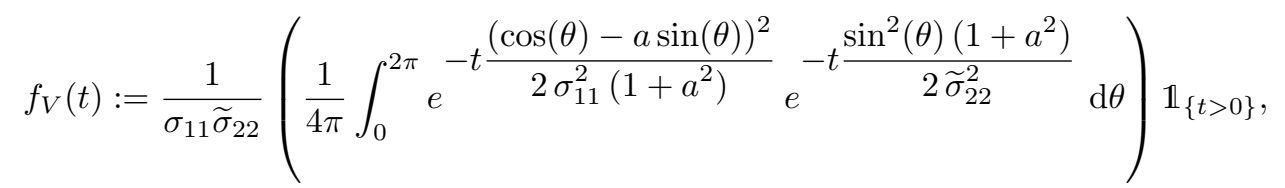


where the coefficients $a$ and $\widetilde{\sigma}_{22}$ are defined by:

$$
a:=\frac{\sigma_{12}}{\sigma_{11}^{2}}, \widetilde{\sigma}_{22}^{2}:=\frac{\sigma_{11}^{2} \sigma_{22}^{2}-\sigma_{12}^{2}}{\sigma_{11}^{2}},
$$

while coefficients $\sigma_{11}, \sigma_{22}$ and $\sigma_{12}$ are

$$
\sigma_{11}^{2}:=4\left(\frac{\pi}{2}\right)^{2} \Sigma_{11}^{(\star \star)}(u), \sigma_{22}^{2}:=\left(\frac{\pi}{2}\right)^{2} \Sigma_{22}^{(\star \star)}(u), \sigma_{12}:=2\left(\frac{\pi}{2}\right)^{2} \Sigma_{12}^{(\star \star)}(u),
$$

with

$$
\Sigma^{(\star \star)}(u)=\left(\Sigma_{i j}^{(\star \star)}(u)\right)_{1 \leqslant i, j \leqslant 2}:=\Sigma_{Q}^{\star}\left(u, \tau, \tau, I_{2}\right)(u),
$$

where $\tau$ is the common value of the eigenvalues of matrix $A$ under the isotropic hypothesis.

Remark 6.3. If $\lambda \neq 1$, by part one of Theorem 6.2 , we readily get that $2 n\left(1-\widehat{\lambda}_{n}\right)$ converges in law to Gaussian random variable with positive infinite mean when $n$ tends to infinity. Thus one gets a one more way to detect if the process is isotropic or not.

Remark 6.4. By Remark 5.14, the matrix $\Sigma^{(\star \star)}(u)$ is non-degenerate, ensuring that the coefficient $\widetilde{\sigma}_{22}$ appearing in the expression of the density $f_{V}$ does not vanish.

Remark 6.5. We suppose that the covariance function $r_{z}$ of $Z$ is known. We can estimate the density $f_{V}$ by $\widehat{f}_{V}(t):=f_{V^{\prime}}\left(\widehat{\tau}_{n}^{2} t\right) \widehat{\tau}_{n}^{2}$, where $\widehat{\tau}_{n}$ is a consistent estimator of $\tau$, the common value of the eigenvalues of matrix $A$ under the isotropic case and where $V^{\prime}$ has the same law as that of $V$, where we replaced $\Sigma^{(\star \star)}(u)$ by $Q \Sigma_{\star}\left(u, 1, I_{2}\right) Q^{t}$, where $\Sigma_{\star}$ being defined in equality (5.12) is computable. By using convergence in (5.13) we can take for $\widehat{\tau}_{n}$,

$$
\widehat{\tau}_{n}:=2 J_{1}^{(n)}(u) \sqrt{\frac{r_{z}(0)}{\mu}} \exp \left(\frac{1}{2} \frac{u^{2}}{r_{z}(0)}\right),
$$

where we recall that $\mu=-\frac{\partial^{2} r_{z}}{\partial t_{i}^{2}}(0)$, for any $i=1,2$.

Proof of Theorem 6.2. Let us look first at the case where $0<\lambda<1$ and $\theta=\frac{\pi}{2}$, then we will treat the case where $\lambda=1$ (and $-\frac{\pi}{2}<\theta \leqslant \frac{\pi}{2}$ ).

In the first case, by decomposition obtained in (6.3) in the proof of Theorem 6.1, we have

$$
\begin{aligned}
& 2 n\left(X_{n}-X\right)\left(X_{n}+X\right) {\left[I^{4}\left(\widehat{\lambda}_{n}\right)\left(X_{n}^{2}+Y_{n}^{2}\right)+X^{2} I^{4}\left(\widehat{\lambda}_{n}\right)-I^{2}\left(\widehat{\lambda}_{n}\right)\left(\widehat{\lambda}_{n}^{2}+1\right)\right] } \\
&+ 2 n Y_{n}^{2} I^{4}\left(\widehat{\lambda}_{n}\right) X^{2}-2 n\left(\lambda I\left(\widehat{\lambda}_{n}\right)-I(\lambda) \widehat{\lambda}_{n}\right) \times \\
& \frac{1}{I^{4}(\lambda)}\left(I^{2}(\lambda)-\lambda^{2} I^{2}\left(\widehat{\lambda}_{n}\right)\right)\left(\lambda I\left(\widehat{\lambda}_{n}\right)+I(\lambda) \widehat{\lambda}_{n}\right)=0,
\end{aligned}
$$

where

$$
\left(X_{n}, Y_{n}\right) \underset{n \rightarrow+\infty}{\stackrel{a . s .}{\longrightarrow}}(X, Y)=(\lambda / I(\lambda), 0) .
$$

Now by using this almost sure convergence result and the one given in (5.11) plus Theorem 6.1, one obtains the following probability equivalence

$$
2 n\left(X_{n}-X\right) 2 \lambda I(\lambda)\left(\lambda^{2}-1\right) \simeq 2 n\left(\lambda I\left(\widehat{\lambda}_{n}\right)-I(\lambda) \widehat{\lambda}_{n}\right) \frac{2 \lambda}{I(\lambda)}\left(1-\lambda^{2}\right),
$$


and since $0<\lambda<1$, one finally gets

$$
2 n\left(\lambda I\left(\widehat{\lambda}_{n}\right)-I(\lambda) \widehat{\lambda}_{n}\right) \simeq-2 n\left(X_{n}-X\right) I^{2}(\lambda) .
$$

Now refering to function $f$ defined by (6.4) in the proof of Theorem 6.1, let

$$
f(x)=\lambda I(x)-I(\lambda) x, \text { for } 0 \leqslant x \leqslant 1 .
$$

One has $f(\lambda)=0$ and $f^{\prime}(\lambda)=\lambda I^{\prime}(\lambda)-I(\lambda)<0$.

So with the first order Taylor expansion of $f$ about $\lambda$ evaluated at $\widehat{\lambda}_{n}$, one gets

$$
\begin{aligned}
2 n\left(\lambda I\left(\widehat{\lambda}_{n}\right)-I(\lambda) \widehat{\lambda}_{n}\right) & =2 n f\left(\widehat{\lambda}_{n}\right) \\
& \simeq 2 n f^{\prime}(\lambda)\left(\widehat{\lambda}_{n}-\lambda\right) \\
& \simeq-2 n\left(X_{n}-X\right) I^{2}(\lambda),
\end{aligned}
$$

and then one proved that

$$
2 n\left(\widehat{\lambda}_{n}-\lambda\right) \simeq \frac{2 n\left(X_{n}-X\right) I^{2}(\lambda)}{I(\lambda)-\lambda I^{\prime}(\lambda)}
$$

and by convergence given in (5.11),

$$
2 n\left(\widehat{\lambda}_{n}-\lambda\right) \underset{n \rightarrow+\infty}{\stackrel{L a w}{\longrightarrow}} \mathcal{N}(0 ; \Sigma),
$$

where $\Sigma:=\frac{I^{4}(\lambda)}{\left(I(\lambda)-\lambda I^{\prime}(\lambda)\right)^{2}}\left(\Sigma_{Q}^{\star}(u)\right)_{11}$.

To end the first part of this proof we just have to check that $\left(\Sigma_{\lambda, \frac{\pi}{2}}(u)\right)_{11}=\Sigma$. In this aim, remember that $\Sigma_{\lambda, \frac{\pi}{2}}(u)=C\left(\lambda, \frac{\pi}{2}\right) \times \Sigma_{Q}^{\star}(u) \times C^{t}\left(\lambda, \frac{\pi}{2}\right)$ with

$$
C\left(\lambda, \frac{\pi}{2}\right)=\frac{1}{J_{\vec{F}}\left(\lambda, \frac{\pi}{2}\right)}\left(\begin{array}{cc}
\frac{\partial F_{2}}{\partial \theta}\left(\lambda, \frac{\pi}{2}\right) & -\frac{\partial F_{1}}{\partial \theta}\left(\lambda, \frac{\pi}{2}\right) \\
- & -
\end{array}\right) .
$$

Using Lemma A.3, one obtains that $J_{\vec{F}}\left(\lambda, \frac{\pi}{2}\right)=\frac{\left(\lambda^{2}-1\right)}{\lambda I(\lambda)} \times\left(\frac{\lambda I^{\prime}(\lambda)-I(\lambda)}{I^{2}(\lambda)}\right)$ and

$$
C\left(\lambda, \frac{\pi}{2}\right)=\frac{1}{J_{\vec{F}}\left(\lambda, \frac{\pi}{2}\right)}\left(\begin{array}{cc}
\frac{1-\lambda^{2}}{\lambda I(\lambda)} & 0 \\
- & -
\end{array}\right) .
$$

In this way, one proved that $\left(\Sigma_{\lambda, \frac{\pi}{2}}(u)\right)_{11}=\Sigma$, yielding the first part of theorem. Now we consider the second part taking $\lambda=1$ (and $-\frac{\pi}{2}<\theta \leqslant \frac{\pi}{2}$ ).

The decomposition obtained in (6.1) gives

$$
\begin{aligned}
& 2 n\left(X_{n}-\frac{2}{\pi}\right)\left(X_{n}+\frac{2}{\pi}\right) \times \\
& 2 n\left[I^{4}\left(\widehat{\lambda}_{n}\right)\left(X_{n}^{2}+Y_{n}^{2}\right)+\left(\frac{2}{\pi}\right)^{2} I^{4}\left(\widehat{\lambda}_{n}\right)-I^{2}\left(\widehat{\lambda}_{n}\right)\left(\widehat{\lambda}_{n}^{2}+1\right)\right] \\
& +\left(\frac{2}{\pi}\right)^{2} I^{4}\left(\widehat{\lambda}_{n}\right)\left(2 n Y_{n}\right)^{2} \\
& =(2 n)^{2}\left(\frac{\pi}{2}-I\left(\widehat{\lambda}_{n}\right)\right)\left(I\left(\widehat{\lambda}_{n}\right)-\frac{\pi}{2} \widehat{\lambda}_{n}\right)\left(\frac{2}{\pi}\right)^{4}\left(\frac{\pi}{2}+I\left(\widehat{\lambda}_{n}\right)\right)\left(I\left(\widehat{\lambda}_{n}\right)+\frac{\pi}{2} \widehat{\lambda}_{n}\right)
\end{aligned}
$$

where

$$
\left(X_{n}, Y_{n}\right) \underset{n \rightarrow+\infty}{\stackrel{a . s .}{\longrightarrow}}(X, Y)=\left(\frac{2}{\pi}, 0\right)
$$


Note that we can not hold the same reasoning as in the first part of the proof. Indeed, by the first part of Theorem 6.1 and the latter almost sure convergence result, we deduce that

$$
Z_{n}:=I^{4}\left(\widehat{\lambda}_{n}\right)\left(X_{n}^{2}+Y_{n}^{2}\right)+\left(\frac{2}{\pi}\right)^{2} I^{4}\left(\widehat{\lambda}_{n}\right)-I^{2}\left(\widehat{\lambda}_{n}\right)\left(\widehat{\lambda}_{n}^{2}+1\right) \underset{n \rightarrow+\infty}{\stackrel{a . s .}{\longrightarrow}} 0
$$

thus we have to normalize the studied expression by $(2 n)^{2}$.

However, if in a first time we do not normalize this expression by $(2 n)^{2}$ but rather by $(2 n)$, we get

$$
\begin{gathered}
2 n\left(X_{n}-\frac{2}{\pi}\right)\left(X_{n}+\frac{2}{\pi}\right) Z_{n}+\left(\frac{2}{\pi}\right)^{2} I^{4}\left(\widehat{\lambda}_{n}\right) 2 n Y_{n}^{2} \\
=2 n h\left(\widehat{\lambda}_{n}\right)\left(\frac{2}{\pi}\right)^{4}\left(\frac{\pi}{2}+I\left(\widehat{\lambda}_{n}\right)\right)\left(I\left(\widehat{\lambda}_{n}\right)+\frac{\pi}{2} \widehat{\lambda}_{n}\right),
\end{gathered}
$$

remembering that the function $h$ (see (6.2)) has been defined in the proof of Theorem 6.1 by

$$
h(\lambda)=\left(\frac{\pi}{2}-I(\lambda)\right)\left(I(\lambda)-\frac{\pi}{2} \lambda\right)
$$

for $0<\lambda \leqslant 1$.

Now by using the almost sure convergence to 0 for $Z_{n}$ and those given in (5.11) and (6.7) and in the first part of Theorem 6.1, we get

$$
2 n h\left(\widehat{\lambda}_{n}\right) \underset{n \rightarrow+\infty}{\stackrel{P}{\longrightarrow}} 0
$$

the convergence taking place in probability.

Since $h(1)=h^{\prime}(1)=0$, one obtains by using a second order Taylor expansion of $h$ about 1 evaluated at point $\widehat{\lambda}_{n}$

$$
h\left(\widehat{\lambda}_{n}\right)=\frac{\pi^{2}}{16}\left(\widehat{\lambda}_{n}-1\right)^{2}+o\left(\left(\widehat{\lambda}_{n}-1\right)^{2}\right) .
$$

Thus as a bonus we proved that in probability

$$
2 n\left(\widehat{\lambda}_{n}-1\right)^{2}=o(1) .
$$

This last equality will help us to study the normalized expression $2 n Z_{n}$ and show that in probability

$$
2 n Z_{n} \simeq 2 n\left(X_{n}-\frac{2}{\pi}\right) \frac{\pi^{3}}{4}
$$

Indeed

$$
\begin{aligned}
2 n Z_{n}=I^{2}\left(\widehat{\lambda}_{n}\right)[2 n & \left(X_{n}^{2}-\left(\frac{2}{\pi}\right)^{2}\right) I^{2}\left(\widehat{\lambda}_{n}\right)+2 n I^{2}\left(\widehat{\lambda}_{n}\right) Y_{n}^{2} \\
& \left.+2 n\left(2 I^{2}\left(\widehat{\lambda}_{n}\right)\left(\frac{2}{\pi}\right)^{2}-\left(\widehat{\lambda}_{n}^{2}+1\right)\right)\right] .
\end{aligned}
$$

Using an order two Taylor expansion of the elliptic integral $I$ (see (5.3) for definition) in $\widehat{\lambda}_{n}$ about $\lambda=1$, one gets

$$
I\left(\widehat{\lambda}_{n}\right)=\frac{\pi}{2}+\frac{\pi}{4}\left(\widehat{\lambda}_{n}-1\right)+O\left(\left(\widehat{\lambda}_{n}-1\right)^{2}\right)
$$

in such a way that

$$
2 n\left(2 I^{2}\left(\widehat{\lambda}_{n}\right)\left(\frac{2}{\pi}\right)^{2}-\left(\widehat{\lambda}_{n}^{2}+1\right)\right)=-n\left(\widehat{\lambda}_{n}-1\right)^{2}+O\left(2 n\left(\widehat{\lambda}_{n}-1\right)^{2}\right) .
$$


By equality (6.9) this expression converges in probability to zero.

Part one of Theorem 6.1, convergences in (5.11) and (6.7) give the required result

$$
2 n Z_{n} \simeq 2 n\left(X_{n}-\frac{2}{\pi}\right) \frac{\pi^{3}}{4} .
$$

Back to expression (6.6), using once again the convergence obtained in part one of Theorem 6.1 and equality (6.8), one finally proved that in probability

$$
\pi^{2}\left(2 n\left(X_{n}-\frac{2}{\pi}\right)\right)^{2}+\left(2 n Y_{n}\right)^{2}\left(\frac{\pi}{2}\right)^{2} \simeq(2 n)^{2} h\left(\widehat{\lambda}_{n}\right) \frac{16}{\pi^{2}} \simeq\left(2 n\left(\widehat{\lambda}_{n}-1\right)\right)^{2} .
$$

By using convergence given in (5.11), we get the required convergence. This ends the proof of this theorem.

Proof of Remark 6.5. The density $f_{V}$ of the positive random variable $V$ can be expressed as $f_{V}(t)=f_{V^{\prime}}\left(t \tau^{2}\right) \tau^{2}$, where $\tau$ is the common eigenvalue of matrix $A$ under the isotropic case and $V^{\prime}$ is defined as $V$, substituting $Q \Sigma_{\star}\left(u, 1, I_{2}\right) Q^{t}$ to $\Sigma^{\star}\left(u, \tau, \tau, I_{2}\right)$. The matrix $\Sigma_{\star}$ is computable and is defined by (5.12). So, it is enough to estimate $\tau$ by a consistent estimator, say $\widehat{\tau}_{n}$. Using convergence given in (5.13) we propose a consistent estimator of $\lambda_{1}=\tau$ in the isotropic case by taking

$$
\left.\widehat{\tau}_{n}:=\frac{J_{1}^{(n)}(u)}{p_{Z(0)}(u) \mathbb{E}\left[\|\nabla Z(0)\|_{2}\right.}\right]=2 J_{1}^{(n)}(u) \sqrt{\frac{r_{z}(0)}{\mu}} \exp \left(\frac{1}{2} \frac{u^{2}}{r_{z}(0)}\right),
$$

that is computable since $\mu$ and $r_{z}(0)$ are supposed to be known.

In the next section, statistical tests are proposed for the null hypothesis " $X$ is isotropic" against " $X$ is affine".

\subsection{Testing the isotropy}

We test

$$
H_{0}: \lambda=1 \quad \text { against } \quad H_{1}: \lambda<1 \text {. }
$$

We still obtain a way to detect the possible isotropy of the process via the following corollaries.

Corollary 6.6. Under the hypothesis $H_{0}$, the following convergence holds:

$$
\frac{J_{\overrightarrow{f^{\star}}}^{(n)}(u)}{J_{1}^{(n)}(u)} \underset{n \rightarrow+\infty}{\stackrel{a . s .}{\longrightarrow}} \frac{2}{\pi} v^{\star}
$$

Corollary 6.7. Under the hypothesis $H_{0}$, we have the following convergence:

$$
T_{\overrightarrow{f^{\star}}}^{(n)}(u):=2 n\left(\frac{J_{\overrightarrow{f^{\star}}}^{(n)}(u)}{J_{\mathbf{1}}^{(n)}(u)}-\frac{2}{\pi} v^{\star}\right) \underset{n \rightarrow+\infty}{\stackrel{L a w}{\longrightarrow}} \mathcal{N}\left(0 ; \Sigma^{\star}\left(u, \tau, \tau, I_{2}\right)\right),
$$

where $\tau$ is the common value of the eigenvalues of matrix $A$.

Remark 6.8. By Remark 5.14 we can observe that the matrix $\Sigma^{\star}\left(u, \tau, \tau, I_{2}\right)$ is nondegenerate.

Remark 6.9. Under the alternative hypothesis $H_{1}$, the test statistic $T_{\overrightarrow{f^{\star}}}^{(n)}(u)$ converges in law towards a Gaussian random variable, the mean of which tends to infinity. Indeed by using equality (5.4) one can easily show that

$$
\left(\frac{\mathbb{E}\left[J_{\overrightarrow{f^{\star}}}^{(1)}(u)\right]}{\mathbb{E}\left[J_{\mathbf{1}}^{(1)}(u)\right]}=\frac{2}{\pi} v^{\star}\right) \Longleftrightarrow(\lambda=1) .
$$


Proof of Corollaries 6.6 and 6.7. Using the almost sure convergence given in Proposition 5.2 , convergence in law of Proposition 5.12, equality (5.4) and taking $\lambda=1$ one obviously gets Corollaries 6.6 and 6.7.

Proof of Remark 6.9. Appling Proposition 5.12, under $H_{1}$, the test statistic $T_{\overrightarrow{f^{\star}}}^{(n)}(u)$ converges in law to a Gaussian random variable with asymptotically mean equivalent to $2 n\left(\frac{\mathbb{E}\left[J_{f^{\star}}^{(1)}(u)\right]}{\mathbb{E}\left[J_{1}^{(1)}(u)\right]}-\frac{2}{\pi} v^{\star}\right)$.

Since $\left(\frac{\mathbb{E}\left[J_{\vec{f}}^{(1)}(u)\right]}{\mathbb{E}\left[J_{1}^{(1)}(u)\right]}-\frac{2}{\pi} v^{\star}\right)$ does not depend on $n$ and is equal to zero if and if $\lambda=1$, this argument ends the proof of the remark.

In the case where covariance function $r_{z}$ is known, Corollary 6.7 suggests another test statistic. More precisely, let $R$ an unitary matrix obtained by diagonalizing the computable matrix $\Sigma_{\star}\left(u, 1, I_{2}\right)$ defined in (5.12). That is $\Sigma_{\star}\left(u, 1, I_{2}\right)=R \Gamma_{\star} R^{t}$.

We consider the following test statistic $S_{f^{\star}}^{(n)}(u)$ :

$$
S_{\overrightarrow{f^{\star}}}^{(n)}(u):=\widehat{\tau}_{n} \Gamma_{\star}^{-\frac{1}{2}} R^{t} \cdot T_{\overrightarrow{f^{\star}}}^{(n)}(u),
$$

where $\widehat{\tau}_{n}$ is given by (6.5).

We can state the following theorem.

Theorem 6.10. Under the hypothesis $H_{0}$, we have the following convergence:

$$
\Xi_{\overrightarrow{f^{\star}}}^{(n)}(u):=\left(S_{\overrightarrow{f^{\star}}}^{(n)}(u)\right)^{t} S_{\overrightarrow{f^{\star}}}^{(n)}(u) \underset{n \rightarrow+\infty}{\stackrel{L a w}{\longrightarrow}} \chi_{2}^{2} .
$$

Remark 6.11. The rejection region is then $\Xi_{\frac{f^{\star}}{(n)}}^{(u)}>\gamma$. This critical region provides a consistent test for any positive constant $\gamma$, because $\Xi_{f^{\rightleftarrows}}^{(n)}(u)$ is stochastically unbounded, for $n \rightarrow+\infty$, except under the null hypothesis. In fact when $\lambda<1$, $\frac{1}{(2 n)^{2}} \Xi_{\frac{f^{\star}}{(n)}}(u)$ converges in probability to $b>0$, and this implies that $\Xi_{f^{\star}}^{(n)}(u)$ converges in probability to $+\infty$.

Proof of Theorem 6.10 and Remark 6.11. As we have already pointed out in Remark 6.8, the matrix $\Sigma^{\star}\left(u, \tau, \tau, I_{2}\right)$ is invertible and we can factorize $\Sigma_{\star}\left(u, 1, I_{2}\right)$ into $\Sigma_{\star}\left(u, 1, I_{2}\right)=$ $R \Gamma_{\star} R^{t}$, where $R$ is an unitary matrix. Let

$$
S_{\overrightarrow{f^{\star}}}^{(n)}(u)=\widehat{\tau}_{n} \Gamma_{\star}^{-\frac{1}{2}} R^{t} \cdot T_{\overrightarrow{f^{\star}}}^{(n)}(u) .
$$

Since $\widehat{\tau}_{n}$ is a consistent estimator of $\tau$ under hypothesis $H_{0}$, by Corollary 6.7, asymptotically this random vector is a standard Gaussian one and Theorem 6.10 ensues.

To achieve the proof of Remark 6.11, let us check that $\frac{1}{(2 n)^{2}} \Xi_{f^{\star}}^{(n)}(u)$ converges in probability to $b>0$ under the alternative hypothesis $H_{1}$ that is when $\lambda<1$.

In this aim, let us note

$$
\widetilde{S}_{\overrightarrow{f^{\star}}}^{(n)}(u):=\widehat{\tau}_{n} \Gamma_{\star}^{-\frac{1}{2}} R^{t} \cdot \widetilde{T}_{\overrightarrow{f^{\star}}}^{(n)}(u),
$$

where

$$
\widetilde{T}_{\overrightarrow{f^{\star}}}^{(n)}(u):=2 n\left(\frac{J_{\overrightarrow{f^{\star}}}^{(n)}(u)}{J_{\mathbf{1}}^{(n)}(u)}-\frac{\mathbb{E}\left[J_{\overrightarrow{f^{\star}}}^{(1)}(u)\right]}{\mathbb{E}\left[J_{\mathbf{1}}^{(1)}(u)\right]}\right)
$$


We have the following decomposition

$$
\begin{aligned}
\frac{1}{(2 n)^{2}} \Xi_{\overrightarrow{f^{\star}}}^{(n)}(u)= & \frac{1}{(2 n)^{2}}\left(S_{\overrightarrow{f^{\star}}}^{(n)}(u)\right)^{t} S_{\overrightarrow{f^{\star}}}^{(n)}(u) \\
= & \frac{1}{(2 n)^{2}}\left(S_{\overrightarrow{f^{\star}}}^{(n)}(u)-\widetilde{S}_{\overrightarrow{f^{\star}}}^{(n)}(u)\right)^{t}\left(S_{\overrightarrow{f^{\star}}}^{(n)}(u)-\widetilde{S}_{\overrightarrow{f^{\star}}}^{(n)}(u)\right) \\
& +\frac{2}{(2 n)^{2}}\left(\widetilde{S}_{\overrightarrow{f^{\star}}}^{(n)}(u)\right)^{t}\left(S_{\overrightarrow{f^{\star}}}^{(n)}(u)-\widetilde{S}_{\overrightarrow{f^{\star}}}^{(n)}(u)\right) \\
& +\frac{1}{(2 n)^{2}}\left(\widetilde{S}_{\overrightarrow{f^{\star}}}^{(n)}(u)\right)^{t}\left(\widetilde{S}_{\overrightarrow{f^{\star}}}^{(n)}(u)\right) \\
= & (1)+(2)+(3) .
\end{aligned}
$$

Let us look at the third term (3).

$$
(3):=\left(\frac{1}{2 n}\left(\widetilde{T}_{\overrightarrow{f^{\star}}}^{(n)}(u)\right)^{t}\right) \cdot\left(\widehat{\tau}_{n}^{2} R \Gamma_{\star}^{-1} R^{t}\right) \cdot\left(\frac{1}{2 n} \widetilde{T}_{\overrightarrow{f^{\star}}}^{(n)}(u)\right) \underset{n \rightarrow+\infty}{\stackrel{\mathbb{P}}{\longrightarrow}} 0,
$$

using Proposition 5.12 and the fact that $\widehat{\tau}_{n}$ almost surely converges to $\sqrt{\frac{2}{\pi \mu}} \mathbb{E}\left[\|\nabla X(0)\|_{2}\right]$ (see Theorem 4.1 and Proposition 3.10).

For the second term (2).

$$
(2):=\left(\frac{1}{n}\left(\widetilde{T}_{\overrightarrow{f^{\star}}}^{(n)}(u)\right)^{t}\right) \cdot\left(\widehat{\tau}_{n}^{2} R \Gamma_{\star}^{-1} R^{t}\right) \cdot\left(\frac{\mathbb{E}\left[J_{\overrightarrow{f^{\star}}}^{(1)}(u)\right]}{\mathbb{E}\left[J_{1}^{(1)}(u)\right]}-\frac{2}{\pi} v^{\star}\right),
$$

and this term tends in probability towards zero as for the third term (3). Finally, the first term (1) gives

$$
\begin{aligned}
(1):= & \left(\widehat{\tau}_{n} \Gamma_{\star}^{-\frac{1}{2}} R^{t} \cdot\left(\frac{\mathbb{E}\left[J_{f^{\star}}^{(1)}(u)\right]}{\mathbb{E}\left[J_{\mathbf{1}}^{(1)}(u)\right]}-\frac{2}{\pi} v^{\star}\right)\right)^{t} \times \\
& \left(\widehat{\tau}_{n} \Gamma_{\star}^{-\frac{1}{2}} R^{t} \cdot\left(\frac{\mathbb{E}\left[J_{\overrightarrow{f^{\star}}}^{(1)}(u)\right]}{\mathbb{E}\left[J_{\mathbf{1}}^{(1)}(u)\right]}-\frac{2}{\pi} v^{\star}\right)\right)=b_{n},
\end{aligned}
$$

and

$$
\begin{aligned}
\lim _{n \rightarrow+\infty} b_{n}=b:=\frac{2}{\pi \mu}\left(\mathbb{E}\left[\|\nabla X(0)\|_{2}\right]\right)^{2} & \left(\Gamma_{\star}^{-\frac{1}{2}} R^{t} \cdot\left(\frac{\mathbb{E}\left[J_{\overrightarrow{f^{\star}}}^{(1)}(u)\right]}{\mathbb{E}\left[J_{\mathbf{1}}^{(1)}(u)\right]}-\frac{2}{\pi} v^{\star}\right)\right)^{t} \times \\
& \left(\Gamma_{\star}^{-\frac{1}{2}} R^{t} \cdot\left(\frac{\mathbb{E}\left[J_{\overrightarrow{f^{\star}}}^{(1)}(u)\right]}{\mathbb{E}\left[J_{\mathbf{1}}^{(1)}(u)\right]}-\frac{2}{\pi} v^{\star}\right)\right)^{(1)} \geqslant 0 .
\end{aligned}
$$

Since $\Gamma_{\star}^{-\frac{1}{2}} R^{t}$ is invertible, one has

$$
(b>0) \Longleftrightarrow\left(\left(\frac{\mathbb{E}\left[J_{\overrightarrow{f^{\star}}}^{(1)}(u)\right]}{\mathbb{E}\left[J_{1}^{(1)}(u)\right]}-\frac{2}{\pi} v^{\star}\right) \neq 0\right) \Longleftrightarrow(\lambda<1) .
$$

That ends the proof of remark. 


\section{A Appendix}

Proof of Lemma 3.2. Let $F: T \times\left(\mathbb{R}^{2}\right)^{\star} \rightarrow \mathbb{R}$ be a function defined by $F(t, z):=H(t, z$, $\left.\frac{z}{\|z\|_{2}}\right)$, where $H: T \times\left(\mathbb{R}^{2}\right)^{\star} \times S^{1}$ is a measurable bounded continuous function of its arguments except maybe on $T \times\left(\mathbb{R}^{2}\right)^{\star} \times \cup_{i=1}^{\ell}\left\{x_{i}\right\}$, where $x_{i}, i=1, \cdots, \ell$, are a finite number of fixed points of the sphere $S^{1}$. We want to prove that $u \mapsto \mathbb{E}[I(u)]^{2}$ is a continuous function, where for $u \in \mathbb{R}$, we have noted $I(u):=\int_{\mathcal{C}(u)} F(t, \nabla X(t)) \mathbb{1}_{\mathrm{D}_{\mathrm{X}}^{\mathrm{r}}}(t) \mathrm{d} \sigma_{1}(t)$. By Remark 2.13, almost surely $I(u)=\int_{\mathcal{C}(u)} F(t, \nabla X(t)) \mathbb{1}_{\mathrm{D}_{\mathrm{X}}^{\mathrm{r}}}(t) \prod_{i=1}^{\ell} \mathbb{1}_{\left\{\nu_{X}(t) \neq x_{i}\right\}} \mathrm{d} \sigma_{1}(t)$. Also we would like to show a second order Rice formula for the functionnal $I(u)$. Due to the presence of the indicator, function $t \mapsto F(t, \nabla X(t)) \mathbb{1}_{D_{\mathrm{X}}^{\mathrm{r}}}(t) \prod_{i=1}^{\ell} \mathbb{1}_{\left\{\nu_{X}(t) \neq x_{i}\right\}}$ is not continuous on $T$, so we can not apply the two-order Rice formula (2.4) given in Corollary 2.8.

Thus we consider the following approximation. For $m \in \mathbb{N}^{\star}$, let

$$
I_{m}(u):=\int_{\mathcal{C}(u)} G_{m}(t, \nabla X(t)) \mathrm{d} \sigma_{1}(t),
$$

where function $G_{m}: T \times \mathbb{R}^{2} \rightarrow \mathbb{R}$, is defined by

$$
G_{m}(t, z):=\varphi\left(\frac{1}{m\|z\|_{2}}\right) F(t, z) \mathbb{1}_{\{z \neq 0\}} \prod_{i=1}^{\ell} \varphi\left(\frac{1}{m\|z /\| z\left\|_{2}-x_{i}\right\|_{2}}\right) \mathbb{1}_{\left\{\left(z /\|z\|_{2}\right) \neq x_{i}\right\}},
$$

where $\varphi$ has been defined in the proof of Theorem 3.1.

As in the proof of last cited theorem we can prove that function $G_{m}$ is a bounded continuous function on $T \times \mathbb{R}^{2}$.

The two order Rice formula ensues by applying Corollary 2.8 to the functional $I_{m}(u)$. That is for all $u \in \mathbb{R}$, one has

$$
\begin{aligned}
& \mathbb{E}\left[I_{m}(u)\right]^{2}= \\
& \int_{T \times T} \mathbb{E}\left[G_{m}\left(t_{1}, \nabla X\left(t_{1}\right)\right) G_{m}\left(t_{2}, \nabla X\left(t_{2}\right)\right)\left\|\nabla X\left(t_{1}\right)\right\|_{2}\left\|\nabla X\left(t_{2}\right)\right\|_{2} \mid X\left(t_{1}\right)=X\left(t_{2}\right)=u\right] \\
& \quad \times p_{X\left(t_{1}\right), X\left(t_{2}\right)}(u, u) \mathrm{d} t_{1} \mathrm{~d} t_{2}<+\infty
\end{aligned}
$$

We observe that the following convergence holds. For all $t \in T$ and $z \in \mathbb{R}$,

$G_{m}(t, z) \underset{m \rightarrow+\infty}{\longrightarrow} F(t, z) \mathbb{1}_{\{z \neq 0\}} \prod_{i=1}^{\ell} \mathbb{1}_{\left\{\left(z /\|z\|_{2}\right) \neq x_{i}\right\}}$. Then we point out that Remark 2.9 following Corollary 2.8 ensures that

$$
\begin{aligned}
\mathbb{E}\left[\sigma_{1}(\mathcal{C}(u))\right]^{2}=\int_{T \times T} \mathbb{E}\left[\left\|\nabla X\left(t_{1}\right)\right\|_{2}\left\|\nabla X\left(t_{2}\right)\right\|_{2} \mid X\left(t_{1}\right)\right. & \left.=X\left(t_{2}\right)=u\right] \\
& \times p_{X\left(t_{1}\right), X\left(t_{2}\right)}(u, u) \mathrm{d} t_{1} \mathrm{~d} t_{2}<+\infty
\end{aligned}
$$

Thus by using the fact that function $G_{m}$ is bounded, an application of the Lebesgue convergence theorem induces that

$$
\begin{aligned}
& \mathbb{E}[I(u)]^{2}= \int_{T \times T} \mathbb{E}\left[F\left(t_{1}, \nabla X\left(t_{1}\right)\right) \mathbb{1}_{D_{\mathrm{X}}^{\mathrm{r}}}\left(t_{1}\right) \prod_{i=1}^{k} \mathbb{1}_{\left\{\nu_{X}\left(t_{1}\right) \neq x_{i} ; \nu_{X}\left(t_{2}\right) \neq x_{i}\right\}} F\left(t_{2}, \nabla X\left(t_{2}\right)\right) \mathbb{1}_{\mathrm{D}_{\mathrm{X}}^{\mathrm{r}}}\left(t_{2}\right) \times\right. \\
&\left.\left\|\nabla X\left(t_{1}\right)\right\|_{2}\left\|\nabla X\left(t_{2}\right)\right\|_{2} \mid X\left(t_{1}\right)=X\left(t_{2}\right)=u\right] \times p_{X\left(t_{1}\right), X\left(t_{2}\right)}(u, u) \mathrm{d} t_{1} \mathrm{~d} t_{2} \\
&= \int_{T \times T} \mathbb{E}\left[F\left(t_{1}, \nabla X\left(t_{1}\right)\right) \mathbb{1}_{\mathrm{D}_{\mathrm{X}}^{\mathrm{r}}\left(t_{1}\right) F\left(t_{2}, \nabla X\left(t_{2}\right)\right) \mathbb{1}_{\mathrm{D}_{\mathrm{X}}^{\mathrm{r}}}\left(t_{2}\right) \times}\right. \\
&\left.\left\|\nabla X\left(t_{1}\right)\right\|_{2}\left\|\nabla X\left(t_{2}\right)\right\|_{2} \mid X\left(t_{1}\right)=X\left(t_{2}\right)=u\right] \times p_{X\left(t_{1}\right), X\left(t_{2}\right)}(u, u) \mathrm{d} t_{1} \mathrm{~d} t_{2}<+\infty .
\end{aligned}
$$


We have therefore established the second order Rice formula for $I(u)$.

We are going to prove that function $u \mapsto \mathbb{E}[I(u)]^{2}$ is continuous. This would achieve the proof of Lemma 3.2.

In this aim, we use the decomposition given in Wschebor [32, p. 60], that is, for $\tau:=t-s$, where $t, s \in T$

$$
\begin{aligned}
\nabla X(0) & =\xi+(X(0) \alpha+X(\tau) \beta) \\
\nabla X(\tau) & =\xi^{\star}-(X(\tau) \alpha+X(0) \beta)
\end{aligned}
$$

where $\xi$ and $\xi^{\star}$ are centred Gaussian vectors taking values in $\mathbb{R}^{2}$, with joint Gaussian distribution, each of them independent of $(X(0), X(\tau))$, such that, if $\nabla r_{x}$ stands for the Jacobian of the $X$ covariance function,

$$
\begin{gathered}
\alpha:=\frac{r_{x}(\tau) \nabla r_{x}(\tau)}{r_{x}^{2}(0)-r_{x}^{2}(\tau)} ; \quad \beta:=-\frac{r_{x}(0) \nabla r_{x}(\tau)}{r_{x}^{2}(0)-r_{x}^{2}(\tau)} \\
\operatorname{Var}(\xi)=\operatorname{Var}\left(\xi^{\star}\right)=-\nabla^{2} r_{x}(0)-\frac{r_{x}(0)}{r_{x}^{2}(0)-r_{x}^{2}(\tau)} \nabla r_{x}(\tau)\left(\nabla r_{x}(\tau)\right)^{t} \\
\operatorname{Cov}\left(\xi, \xi^{\star}\right)=-\nabla^{2} r_{x}(\tau)-\frac{r_{x}(\tau)}{r_{x}^{2}(0)-r_{x}^{2}(\tau)} \nabla r_{x}(\tau)\left(\nabla r_{x}(\tau)\right)^{t}
\end{gathered}
$$

Thus with these notations one has

$$
\mathbb{E}[I(u)]^{2}=\int_{T} \mathrm{~d} s \int_{T} L(u, s, t-s) \mathrm{d} t,
$$

where for $\tau:=t-s$

$$
\begin{aligned}
L(u, s, \tau):= & \exp \left(-\frac{u^{2}}{r_{x}(0)+r_{x}(\tau)}\right) \frac{1}{2 \pi} \frac{1}{\left(r_{x}^{2}(0)-r_{x}^{2}(\tau)\right)^{\frac{1}{2}}} \\
& \times \mathbb{E}\left[F\left(s, \xi-\frac{u}{r_{x}(0)+r_{x}(\tau)} \nabla r_{x}(\tau)\right) \mathbb{1}_{\left\{\xi-\frac{u}{r_{x}(0)+r_{x}(\tau)}\right.} \nabla r_{x}(\tau) \neq 0\right\} \\
& F\left(\tau+s, \xi^{\star}+\frac{u}{r_{x}(0)+r_{x}(\tau)} \nabla r_{x}(\tau)\right) \mathbb{1}_{\left\{\xi^{\star}+\frac{u}{r_{x}(0)+r_{x}(\tau)} \nabla r_{x}(\tau) \neq 0\right\}} \\
& \left\|\xi_{\xi_{x}}(0)+\frac{u}{r_{x}(0)+r_{x}(\tau)} \nabla\right\|_{2}
\end{aligned}
$$

Since $r_{x}^{2}(0)-r_{x}^{2}(t) \neq 0$ for all $t \neq 0$, this implies in force that $r_{x}(0)+r_{x}(t) \neq 0$ for all $t \neq 0$, thus by using that function $F$ is bounded one obtains that

- $u \mapsto L(u, s, t-s)$ is continuous for almost $(s, t) \in T \times T$.

Now let us enunciate a lemma for which a proof can be founded for example in Berzin and Wschebor [11, Lemma 1 (b)].

Lemma A.1. $\exists M>0, \forall t, s \in T,\left(\|t-s\|_{2} \leqslant M \Longrightarrow r_{x}^{2}(0)-r_{x}^{2}(t-s) \geqslant M\|t-s\|_{2}^{2}\right)$

Now let us choose $B>0$ small enough such that for all $\tau:=s-t$, with $s, t \in T$ such that $\|\tau\|_{2} \leqslant B$, one has the following inequalities,

- (1) $r_{x}(0)+r_{x}(\tau) \geqslant \frac{3}{2} r_{x}(0)$

- (2) $r_{x}^{2}(0)-r_{x}^{2}(\tau) \geqslant B\|\tau\|_{2}^{2}$

- (3) $\left\|\nabla r_{x}(\tau)\right\|_{2} \leqslant \mathbf{C}\|\tau\|_{2}$ 
Note that these three inequalities are always possible to implement. Indeed the first inequality just translates the fact that covariance function $r_{x}$ is continuous in zero, the second one comes from Lemma A.1 and the third one from a first order Taylor expansion of $\nabla r_{x}(\cdot)$ about zero.

These inequalities allow showing the following bound on $|L(u, s, \tau)|$.

- $\exists D>0$ such that for all $(s, t) \in T \times T$ and $\tau:=s-t$,

$$
|L(u, s, \tau)| \leqslant D\left(1+u^{2}\right)\left\{\mathbb{1}_{\left\{\|\tau\|_{2} \geqslant B\right\}}+\frac{1}{\|\tau\|_{2}} \mathbb{1}_{\left\{\|\tau\|_{2} \leqslant B\right\}}\right\} .
$$

Before explaining the last inequality, let us remark that since $\int_{T \times T} \frac{\mathrm{d} s \mathrm{~d} t}{\|s-t\|_{2}}<+\infty$, an application of Lebesgue's convergence theorem yields that function $u \mapsto \mathbb{E}[I(u)]^{2}$ is continuous.

Indeed, two cases occur: $\|\tau\|_{2} \geqslant B$ and $\|\tau\|_{2} \leqslant B$. If $\|\tau\|_{2} \geqslant B$, we consider the compact set $K:=\left\{s-t,(s, t) \in \bar{T} \times \bar{T}\right.$ with $\left.\|s-t\|_{2} \geqslant B\right\}$ where we have noted $\bar{T}$ for the closure of $T$. Since $r_{x}$ is continuous on $K$ and $r_{x}^{2}(0)-r_{x}^{2}(v) \neq 0$ for $v \in K$, thus there exists $E>0$ such for all $v \in K, r_{x}^{2}(0)-r_{x}^{2}(v) \geqslant E$ and $r_{x}(0)+r_{x}(v) \geqslant E$, so that $|L(u, s, \tau)| \leqslant D\left(1+u^{2}\right)$. If $\|\tau\|_{2} \leqslant B$, inequalities (2) and (3) give that $\mathbb{E}\left[\|\xi\|_{2}\right] \leqslant \mathbf{C}$ and $\mathbb{E}\left[\left\|\xi^{\star}\right\|_{2}\right] \leqslant \mathbf{C}$ and inequalities (1) and (2) give that $|L(u, s, \tau)| \leqslant D\left(1+u^{2}\right) \frac{1}{\|\tau\|_{2}}$.

That completes the proof of Lemma 3.2.

Remark A.2. The approach chosen to prove Lemma 3.2 does not work for showing that

$$
\left(u_{1}, u_{2}\right) \mapsto \mathbb{E}\left[J_{f}\left(u_{1}\right) J_{f}\left(u_{2}\right)\right]
$$

is continuous. In fact if we try to reproduce the previous proof, we realize that we have to check that for all $u_{1}, u_{2} \in \mathbb{R}$,

$$
\begin{aligned}
& H\left(u_{1}, u_{2}\right):= \\
& \int_{T \times T} \mathbb{E}\left[\left\|\nabla X\left(t_{1}\right)\right\|_{2}\left\|\nabla X\left(t_{2}\right)\right\|_{2} \mid X\left(t_{1}\right)=u_{1}, X\left(t_{2}\right)=u_{2}\right] \times p_{X\left(t_{1}\right), X\left(t_{2}\right)}\left(u_{1}, u_{2}\right) \mathrm{d} t_{1} \mathrm{~d} t_{2}<+\infty .
\end{aligned}
$$

As before, performing a regression, the expectation appearing in the expression of $H\left(u_{1}, u_{2}\right)$ is

$$
\begin{aligned}
\mathbb{E}\left[\left\|\xi+\frac{r_{x}(\tau) \nabla r_{x}(\tau)}{r_{x}^{2}(0)-r_{x}^{2}(\tau)} u_{1}-\frac{r_{x}(0) \nabla r_{x}(\tau)}{r_{x}^{2}(0)-r_{x}^{2}(\tau)} u_{2}\right\|_{2}\right. \\
\left.\times\left\|\xi^{\star}+\frac{r_{x}(0) \nabla r_{x}(\tau)}{r_{x}^{2}(0)-r_{x}^{2}(\tau)} u_{1}-\frac{r_{x}(\tau) \nabla r_{x}(\tau)}{r_{x}^{2}(0)-r_{x}^{2}(\tau)} u_{2}\right\|_{2}\right]
\end{aligned}
$$

and the principal difficulty results in bounding $\frac{\nabla r_{x}(\tau)}{r_{x}^{2}(0)-r_{x}^{2}(\tau)}$ when $\|\tau\|_{2} \leqslant B$. That is why we developped a little more sophisticated approach to prove Theorem 3.1.

Proof of Lemma 3.9. Let $X=\left(X_{i}\right)_{i=1,2,3}$ and $Y=\left(Y_{j}\right)_{j=1,2,3}$ be two centred standard Gaussian vectors in $\mathbb{R}^{3}$ such that for $1 \leqslant i, j \leqslant 3, \mathbb{E}\left[X_{i} Y_{j}\right]=\rho_{i j}$.

Let $\boldsymbol{k}:=\left(k_{1}, k_{2}, k_{3}\right)$ and $\boldsymbol{m}:=\left(m_{1}, m_{2}, m_{3}\right)$ be two vectors of $\mathbb{N}^{3}$. We want to give an explicit formula for $\mathbb{E}\left[\widetilde{H}_{\boldsymbol{k}}(X) \widetilde{H}_{\boldsymbol{m}}(Y)\right]$.

For ease of notations let us set $Y_{j}:=X_{j+3}$ for $j=1,2,3$. 
As in Azaïs and Wschebor [8, p. 269], a straightforward calculation on Gaussian characteristic functions gives

$$
\begin{aligned}
& \mathbb{E}\left[\prod_{i=1}^{6} \exp \left(t_{i} X_{i}-\frac{1}{2} t_{i}^{2}\right)\right]=\exp \left(\sum_{i=1}^{3} \sum_{j=1}^{3} \rho_{i j} t_{i} t_{j+3}\right) \\
&=\sum_{r=0}^{+\infty} \frac{1}{r !}\left(\sum_{i=1}^{3} \sum_{j=1}^{3} \rho_{i j} t_{i} t_{j+3}\right)^{r}=\sum_{r=0}^{+\infty} \sum_{\sum_{i, j} d_{i j}=r} \prod_{i, j=1}^{3} \frac{1}{d_{i j} !}\left(\rho_{i j} t_{i} t_{j+3}\right)^{d_{i j}} \\
&=\sum_{r=0}^{+\infty} \sum_{\sum_{i, j} d_{i j}=r}\left(\prod_{i, j=1}^{3} \frac{\rho_{i j}^{d_{i j}}}{d_{i j} !}\right)\left(\prod_{i=1}^{3} t_{i}^{\sum_{j=1}^{3} d_{i j}}\right)\left(\prod_{j=1}^{3} t_{j+3}^{\sum_{i=1}^{3} d_{i j}}\right) .
\end{aligned}
$$

Now, by definition

$$
\exp \left(t x-\frac{1}{2} t^{2}\right)=\sum_{q=0}^{+\infty} t^{q} \frac{H_{q}(x)}{q !}
$$

thus

$$
\begin{aligned}
& \mathbb{E}\left[\prod_{i=1}^{6} \exp \left(t_{i} X_{i}-\frac{1}{2} t_{i}^{2}\right)\right] \\
& \quad=\sum_{k_{1}=0}^{+\infty} \sum_{k_{2}=0}^{+\infty} \sum_{k_{3}=0}^{+\infty} \sum_{m_{1}=0}^{+\infty} \sum_{m_{2}=0}^{+\infty} \sum_{m_{3}=0}^{+\infty} \frac{t_{1}^{k_{1}}}{k_{1} !} \frac{t_{2}^{k_{2}}}{k_{2} !} \frac{t_{3}^{k_{3}}}{k_{3} !} \frac{t_{4}^{m_{1}}}{m_{1} !} \frac{t_{5}^{m_{2}}}{m_{2} !} \frac{t_{6}^{m_{6}}}{m_{6} !} \mathbb{E}\left[\widetilde{H}_{\boldsymbol{k}}(X) \widetilde{H}_{\boldsymbol{m}}(Y)\right] .
\end{aligned}
$$

Identifying this last equality with (A.1), it follows that $\mathbb{E}\left[\widetilde{H}_{\boldsymbol{k}}(X) \widetilde{H}_{\boldsymbol{m}}(Y)\right]=0$ if $|\boldsymbol{k}| \neq|\boldsymbol{m}|$. In the case where $|\boldsymbol{k}|=|\boldsymbol{m}|$, one gets

$$
\mathbb{E}\left[\widetilde{H}_{\boldsymbol{k}}(X) \widetilde{H}_{\boldsymbol{m}}(Y)\right]=\sum_{\substack{d_{i j} \geqslant 0 \\ \sum_{j} d_{i j}=k_{i} \\ \sum_{i} d_{i j}=m_{j}}} \boldsymbol{k} ! \boldsymbol{m} ! \prod_{1 \leqslant i, j \leqslant 3} \frac{\rho_{i j}^{d_{i j}}}{d_{i j} !},
$$

yielding the lemma.

Proof of Lemma 4.2. Let $f: S^{1} \rightarrow \mathbb{R}$ be a non-negative function that belongs to $\mathcal{B}_{1}$. For $n \in \mathbb{N}^{\star}$, let $\left.T_{n}:=\right] 0, n[\times] 0, n[$. One has

$$
\begin{aligned}
(1) & :=\int_{0}^{n-1} \int_{0}^{n-1} \int_{\mathcal{C}_{[t, t+1[\times[s, s+1[}(u)} f\left(\nu_{X}(x)\right) \mathrm{d} \sigma_{1}(x) \mathrm{d} t \mathrm{~d} s \\
& -\int_{0}^{1} \int_{0}^{1} \int_{\mathcal{C}_{] 0, t[\times] 0, s[}(u)} f\left(\nu_{X}(x)\right) \mathrm{d} \sigma_{1}(x) \mathrm{d} t \mathrm{~d} s \\
& =\int_{0}^{n-1} \int_{0}^{n-1}(H(t+1, s+1)-H(t, s+1)-H(t+1, s)+H(t, s)) \mathrm{d} t \mathrm{~d} s \\
& -\int_{0}^{1} \int_{0}^{1} H(t, s) \mathrm{d} t \mathrm{~d} s,
\end{aligned}
$$

where we recall that we have noted

$$
H(t, s):=\int_{\mathcal{C}_{] 0, t[\times] 0, s[}(u)} f\left(\nu_{X}(x)\right) \mathrm{d} \sigma_{1}(x) .
$$


Thus by making change of variable in the first integral, one obtains

$$
(1)=\int_{n-1}^{n} \int_{n-1}^{n} H(t, s) \mathrm{d} t \mathrm{~d} s-\int_{0}^{1} \int_{n-1}^{n} H(t, s) \mathrm{d} t \mathrm{~d} s-\int_{n-1}^{n} \int_{0}^{1} H(t, s) \mathrm{d} t \mathrm{~d} s .
$$

Since $f$ is a non-negative function then $H(t, s) \geqslant 0$ and we get the following upper bound

$$
\begin{aligned}
(1) \leqslant & \int_{n-1}^{n} \int_{n-1}^{n} H(t, s) \mathrm{d} t \mathrm{~d} s \\
& \leqslant \int_{n-1}^{n} \int_{n-1}^{n} \int_{\mathcal{C}_{] 0, n[\times] 0, n[}(u)} f\left(\nu_{X}(x)\right) \mathrm{d} \sigma_{1}(x) \mathrm{d} t \mathrm{~d} s=\int_{\mathcal{C}_{] 0, n[\times] 0, n[}(u)} f\left(\nu_{X}(x)\right) \mathrm{d} \sigma_{1}(x) .
\end{aligned}
$$

The first inequality of lemma is then achieved. To obtain the second inequality, arguing as previously we obtain the following lower bound

$$
\begin{aligned}
(2):= & \int_{0}^{n+1} \int_{0}^{n+1} \int_{\mathrm{C}_{[t-1, t[\times[s-1, s[}(u)} f\left(\nu_{X}(x)\right) \mathrm{d} \sigma_{1}(x) \mathrm{d} t \mathrm{~d} s=\int_{n}^{n+1} \int_{n}^{n+1} H(t, s) \mathrm{d} t \mathrm{~d} s \\
& -\int_{-1}^{0} \int_{n}^{n+1} H(t, s) \mathrm{d} t \mathrm{~d} s-\int_{n}^{n+1} \int_{-1}^{0} H(t, s) \mathrm{d} t \mathrm{~d} s+\int_{-1}^{0} \int_{-1}^{0} H(t, s) \mathrm{d} t \mathrm{~d} s \\
\geqslant & \int_{n}^{n+1} \int_{n}^{n+1} \int_{\mathrm{C}_{] 0, t[\times] 0, s[}(u)} f\left(\nu_{X}(x)\right) \mathrm{d} \sigma_{1}(x) \mathrm{d} t \mathrm{~d} s \geqslant \int_{\mathcal{C}_{] 0, n[\times] 0, n[}(u)} f\left(\nu_{X}(x)\right) \mathrm{d} \sigma_{1}(x),
\end{aligned}
$$

this yields Lemma 4.2 .

Proof of Lemma 5.4. Since $Z$ is an isotropic process, its density $p_{\nabla Z(0)}(v)$ depends only on $\|v\|_{2}$. We denote it by $g\left(\|v\|_{2}\right)$.

Thus if $\vec{f}: S^{1} \rightarrow \mathbb{R}^{2}$ is a function that belongs to $\mathcal{B}_{2}$,

$$
\begin{aligned}
\mathbb{E}\left[\vec{f}\left(\nu_{X}(0)\right)\|\nabla X(0)\|_{2}\right] & =\mathbb{E}\left[\vec{f}\left(\frac{A . \nabla Z(0)}{\|A \cdot \nabla Z(0)\|_{2}}\right)\|A \cdot \nabla Z(0)\|_{2}\right] \\
& =\int_{\mathbb{R}^{2}} \vec{f}\left(\frac{A \cdot v}{\|A \cdot v\|_{2}}\right)\|A \cdot v\|_{2} g\left(\|v\|_{2}\right) \mathrm{d} v .
\end{aligned}
$$

Letting $v:=r \alpha, \alpha \in S^{1}$, one gets

$$
\begin{aligned}
& \mathbb{E}\left[\vec{f}\left(\nu_{X}(0)\right)\|\nabla X(0)\|_{2}\right] \\
& =\sigma_{1}\left(S^{1}\right)\left(\int_{0}^{\infty} g(r) r^{2} \mathrm{~d} r\right)\left(\int_{S^{1}} \vec{f}\left(\frac{A . \alpha}{\|A \cdot \alpha\|_{2}}\right)\|A \cdot \alpha\|_{2} \mathrm{~d} \alpha\right) \\
& =\mathbb{E}\left[\|\nabla Z(0)\|_{2}\right]\left(\int_{S^{1}} \vec{f}\left(\frac{A \cdot \alpha}{\|A . \alpha\|_{2}}\right)\|A \cdot \alpha\|_{2} \mathrm{~d} \alpha\right) .
\end{aligned}
$$

In a similar way taking $f \equiv \mathbf{1}$, one gets

$$
\mathbb{E}\left[\|\nabla X(0)\|_{2}\right]=\mathbb{E}\left[\|\nabla Z(0)\|_{2}\right]\left(\int_{S^{1}}\|A \cdot \alpha\|_{2} \mathrm{~d} \alpha\right) .
$$

Finally by using Proposition 3.10, one obtains

$$
\frac{\mathbb{E}\left[J_{\vec{f}}^{(n)}(u)\right]}{\mathbb{E}\left[J_{\mathbf{1}}^{(n)}(u)\right]}=\frac{\int_{S^{1}} \vec{f}\left(\frac{A . \alpha}{\|A . \alpha\|_{2}}\right)\|A . \alpha\|_{2} \mathrm{~d} \alpha}{\int_{S^{1}}\|A . \alpha\|_{2} \mathrm{~d} \alpha} .
$$


Now choosing $\vec{f}:=\overrightarrow{f^{\star}}$ defined by (5.1) and since $A$ is a self-adjoint matrix, one has

$$
\begin{aligned}
\int_{S^{1}} \overrightarrow{f^{\star}}\left(\frac{A . \alpha}{\|A \cdot \alpha\|_{2}}\right)\|A . \alpha\|_{2} \mathrm{~d} \alpha=2 \int_{S^{1}} A \cdot \alpha \mathbb{1}_{\left\{\left\langle A . \alpha, v^{\star}\right\rangle \geqslant 0\right\}} \mathrm{d} \alpha \\
=2 \int_{S^{1}} A \cdot \alpha \mathbb{1}_{\left\{\left\langle\alpha, A \cdot v^{\star}\right\rangle \geqslant 0\right\}} \mathrm{d} \alpha=2 A \cdot \int_{S^{1} \cap\left\{\left\langle\alpha, w^{\star}\right\rangle \geqslant 0\right\}} \alpha \mathrm{d} \alpha
\end{aligned}
$$

where we have noted $w^{\star}:=\frac{A \cdot v^{\star}}{\left\|A \cdot v^{\star}\right\|_{2}}$.

If $R$ denotes the change of basis matrix from the canonical orthonormal basis $\mathbf{e}:=(\vec{i}, \vec{j})$ to an orthonormal basis choosen as $\mathbf{w}:=\left(w_{1}^{\star}, w^{\star}\right)$ and making the change of variable $\beta:=R^{t} . \alpha$ in the last integral, one obtains

$$
\begin{aligned}
& \left(\int_{\left.S^{1} \cap\left\{\left\langle\alpha, w^{\star}\right\rangle_{\mathbf{e}} \geqslant 0\right\}^{\alpha \mathrm{d} \alpha}\right)_{\mathbf{e}}} \alpha \mathrm{d} \alpha\right)_{\mathbf{w}}=\left(0,\left(\int_{S^{1} \cap\left\{\alpha_{2} \geqslant 0\right\}} \alpha_{2} \mathrm{~d} \alpha\right)\right)_{\mathbf{w}} \\
& =\left(\int_{S^{1} \cap\left\{\langle\alpha,(0,1)\rangle_{\mathbf{w}} \geqslant 0\right\}} \alpha^{\pi} \mathrm{d} \alpha\right) w^{\star}=\left(\int_{0}^{\pi} \sin (x) \frac{\mathrm{d} x}{2 \pi}\right) w^{\star}=\frac{1}{\pi} w^{\star} .
\end{aligned}
$$

Thus we have proved that

$$
\int_{S^{1}} \overrightarrow{f^{\star}}\left(\frac{A \cdot \alpha}{\|A \cdot \alpha\|_{2}}\right)\|A \cdot \alpha\|_{2} \mathrm{~d} \alpha=\frac{2}{\pi} A \cdot w^{\star}=\frac{2}{\pi} \frac{A^{2} \cdot v^{\star}}{\left\|A \cdot v^{\star}\right\|_{2}},
$$

that yields Lemma 5.4.

Lemma A.3. The Jacobian $J_{\vec{F}}$ of transformation $\vec{F}$ (see (5.5)) is given by

$$
\begin{aligned}
& J_{\vec{F}}(\lambda, \theta)=\frac{\lambda\left(1-\lambda^{2}\right)}{I^{3}(\lambda)\left(\cos ^{2}(\theta)+\lambda^{2} \sin ^{2}(\theta)\right)} \times \\
& {\left[\sin ^{2}(\theta) \int_{0}^{1} \frac{\sqrt{1-u^{2}}}{\sqrt{1-\left(1-\lambda^{2}\right) u^{2}}} \mathrm{~d} u\right.} \\
& \left.+\cos ^{2}(\theta) \int_{0}^{1} \frac{u^{2}}{\sqrt{1-\left(1-\lambda^{2}\right) u^{2}}} \frac{\mathrm{d} u}{\sqrt{1-u^{2}}}\right] \neq 0,
\end{aligned}
$$

for $0<\lambda<1$.

Proof of Lemma A.3. For $0<\lambda \leqslant 1$ and $-\frac{\pi}{2}<\theta \leqslant \frac{\pi}{2}$, one has

$$
\left\{\begin{array}{l}
\frac{\partial F_{1}}{\partial \lambda}(\lambda, \theta)=\frac{1}{I^{2}(\lambda)} \frac{\left[\lambda \sin ^{2}(\theta) I(\lambda)-\left(\cos ^{2}(\theta)+\lambda^{2} \sin ^{2}(\theta)\right) I^{\prime}(\lambda)\right]}{\left(\cos ^{2}(\theta)+\lambda^{2} \sin ^{2}(\theta)\right)^{\frac{1}{2}}} \\
\frac{\partial F_{1}}{\partial \theta}(\lambda, \theta)=\frac{1}{I(\lambda)} \frac{\left(\lambda^{2}-1\right) \sin (\theta) \cos (\theta)}{\left(\cos ^{2}(\theta)+\lambda^{2} \sin ^{2}(\theta)\right)^{\frac{1}{2}}},
\end{array}\right.
$$

and

$$
\left\{\begin{array}{l}
\frac{\partial F_{2}}{\partial \lambda}(\lambda, \theta)=\frac{\sin (\theta) \cos (\theta)}{I^{2}(\lambda)} \frac{\left(\begin{array}{l}
\left(\lambda I(\lambda)-\left(\lambda^{2}-1\right) I^{\prime}(\lambda)\right) \\
\times\left(\cos ^{2}(\theta)+\lambda^{2} \sin ^{2}(\theta)\right) \\
+\lambda I(\lambda)
\end{array}\right)}{\left(\cos ^{2}(\theta)+\lambda^{2} \sin ^{2}(\theta)\right)^{\frac{3}{2}}} \\
\frac{\partial F_{2}}{\partial \theta}(\lambda, \theta)=\frac{\left(\lambda^{2}-1\right)}{I(\lambda)} \frac{\left[\cos ^{4}(\theta)-\lambda^{2} \sin ^{4}(\theta)\right]}{\left(\cos ^{2}(\theta)+\lambda^{2} \sin ^{2}(\theta)\right)^{\frac{3}{2}}}
\end{array}\right.
$$


From straightforward calculations we deduce that the Jacobian $J_{\vec{F}}$ of the transformation $\vec{F}$ can be written as

$$
J_{\vec{F}}(\lambda, \theta)=\frac{\left(1-\lambda^{2}\right)}{I^{3}(\lambda)} \frac{\left[\lambda \sin ^{2}(\theta) I(\lambda)+\left(\cos ^{2}(\theta)-\lambda^{2} \sin ^{2}(\theta)\right) I^{\prime}(\lambda)\right]}{\left(\cos ^{2}(\theta)+\lambda^{2} \sin ^{2}(\theta)\right)}
$$

Now $I(\lambda)=\int_{0}^{\frac{\pi}{2}}\left(\cos ^{2}(x)+\lambda^{2} \sin ^{2}(x)\right)^{\frac{1}{2}} \mathrm{~d} x$, and the change of variable: $\sin (x)=u$ gives $I(\lambda)=\int_{0}^{1} \frac{\left(1-\left(1-\lambda^{2}\right) u^{2}\right)^{\frac{1}{2}}}{\left(1-u^{2}\right)^{\frac{1}{2}}} \mathrm{~d} u$ and $I^{\prime}(\lambda)=\lambda \int_{0}^{1} \frac{u^{2}}{\left(1-\left(1-\lambda^{2}\right) u^{2}\right)^{\frac{1}{2}}} \frac{\mathrm{d} u}{\sqrt{1-u^{2}}}$.

This yields

$$
\begin{aligned}
\lambda \sin ^{2}(\theta) I(\lambda)+\left(\cos ^{2}(\theta)-\lambda^{2} \sin ^{2}(\theta)\right) I^{\prime}(\lambda) & = \\
\lambda & {\left[\sin ^{2}(\theta) \int_{0}^{1} \frac{\sqrt{1-u^{2}}}{\sqrt{1-\left(1-\lambda^{2}\right) u^{2}}} \mathrm{~d} u\right.} \\
& \left.\quad+\cos ^{2}(\theta) \int_{0}^{1} \frac{u^{2}}{\sqrt{1-\left(1-\lambda^{2}\right) u^{2}}} \frac{\mathrm{d} u}{\sqrt{1-u^{2}}}\right],
\end{aligned}
$$

and lemma ensues from the fact that $\left(J_{\vec{F}}(\lambda, \theta)=0\right) \Longleftrightarrow(\lambda=1)$.

Proof of Lemma 5.9. First let us see that for $n \in \mathbb{N}^{\star}$, a.s. $X_{n}^{2}+Y_{n}^{2}<1$.

$$
X_{n}^{2}+Y_{n}^{2}=\frac{\left\|\int_{\mathcal{C}_{n}(u)} \overrightarrow{f^{\star}}\left(\nu_{X}(t)\right) \mathrm{d} \sigma_{1}(t)\right\|_{2}^{2}}{\sigma_{1}^{2}\left(\mathcal{C}_{n}(u)\right)} \leqslant \frac{\int_{\mathcal{C}_{n}(u)}\left\|\overrightarrow{f^{\star}}\left(\nu_{X}(t)\right)\right\|_{2}^{2} \mathrm{~d} \sigma_{1}(t)}{\sigma_{1}\left(\mathcal{C}_{n}(u)\right)}=1,
$$

last equality coming from the fact that $\overrightarrow{f^{\star}}$ takes its values into $S^{1}$. But the strict inequality is true, otherwise we would have equality in Hölder inequality that remains impossible.

Now let us see that, a.s. $X_{n}>0$.

$$
X_{n}=\left\langle\frac{J_{\overrightarrow{f^{\star}}}^{(n)}(u)}{J_{\mathbf{1}}^{(n)}(u)}, v^{\star}\right\rangle=\frac{1}{\sigma_{1}\left(\mathcal{C}_{n}(u)\right)} \int_{\mathcal{C}_{n}(u)}\left|\left\langle\nu_{X}(t), v^{\star}\right\rangle\right| \mathrm{d} \sigma_{1}(t) \geqslant 0,
$$

and in a manner similar to that previously used it is proved that, a.s. $X_{n}>0$. This yields lemma.

Lemma A.4. The coefficients $a_{f_{1}^{\star}}$ are given by: for $m, \ell \in \mathbb{N}$

$$
\begin{array}{r}
\left(\begin{array}{rrrr}
a_{f_{1}^{\star}}(2 m, 2 \ell) & a_{f_{1}^{\star}}(2 m+1,2 \ell+1) & a_{f_{1}^{\star}}(2 m, 2 \ell+1) & a_{f_{1}^{\star}}(2 m+1,2 \ell) \\
a_{f_{2}^{\star}}(2 m, 2 \ell) & a_{f_{2}^{\star}}(2 m+1,2 \ell+1) & a_{f_{2}^{\star}}(2 m, 2 \ell+1) & a_{f_{2}^{\star}}(2 m+1,2 \ell
\end{array}\right)= \\
P \times\left(\begin{array}{lllll}
A\left(\lambda_{1}, \lambda_{2}, \omega_{1}^{\star}, \omega_{2}^{\star}, m, \ell, \mu\right) & B\left(\lambda_{1}, \lambda_{2}, \omega_{1}^{\star}, \omega_{2}^{\star}, m, \ell, \mu\right) & 0 & 0 \\
A\left(\lambda_{2}, \lambda_{1}, \omega_{2}^{\star}, \omega_{1}^{\star}, \ell, m, \mu\right) & B\left(\lambda_{2}, \lambda_{1}, \omega_{2}^{\star}, \omega_{1}^{\star}, \ell, m, \mu\right) & 0 & 0
\end{array}\right),
\end{array}
$$

where $\omega^{\star}=\left(\omega_{1}^{\star}, \omega_{2}^{\star}\right):=v^{\star} \cdot P$ and

$$
\begin{aligned}
& A\left(\lambda_{1}, \lambda_{2}, \omega_{1}^{\star}, \omega_{2}^{\star}, m, \ell, \mu\right):= \\
& \quad \sqrt{\frac{2 \mu}{\pi}} \lambda_{1} \sum_{p=0}^{m} \frac{(-2)^{p-m}}{(2 p) !(m-p) !} \sum_{k=0}^{p} \frac{2^{k} p !}{(p-k) !}\left(\frac{\lambda_{2} \omega_{2}^{\star}}{\lambda_{1} \omega_{1}^{\star}}\right)^{2(p-k)} \times \\
& \sum_{n=0}^{\ell} \frac{(-2)^{n-\ell}}{(2 n) !(\ell-n) !}\left(\frac{\lambda_{1} \omega_{1}^{\star}}{\left[\left(\lambda_{1} \omega_{1}^{\star}\right)^{2}+\left(\lambda_{2} \omega_{2}^{\star}\right)^{2}\right]^{\frac{1}{2}}}\right)^{2(n+p-k)+1} \times \\
& \frac{(2(n+p-k)) !}{2^{n+p-k}(n+p-k) !},
\end{aligned}
$$


and

$$
\begin{gathered}
B\left(\lambda_{1}, \lambda_{2}, \omega_{1}^{\star}, \omega_{2}^{\star}, m, \ell, \mu\right):= \\
\quad \sqrt{\frac{2 \mu}{\pi}} \lambda_{1} \sum_{p=0}^{\ell} \frac{(-2)^{p-\ell}}{(2 p+1) !(\ell-p) !} \sum_{k=0}^{p} \frac{2^{k} p !}{(p-k) !}\left(\frac{\lambda_{1} \omega_{1}^{\star}}{\lambda_{2} \omega_{2}^{\star}}\right)^{2(p-k)} \times \\
\sum_{n=0}^{m} \frac{(-2)^{n-m}}{(2 n+1) !(m-n) !}\left(\frac{\lambda_{2} \omega_{2}^{\star}}{\left[\left(\lambda_{1} \omega_{1}^{\star}\right)^{2}+\left(\lambda_{2} \omega_{2}^{\star}\right)^{2}\right]^{\frac{1}{2}}}\right)^{2(p-k+n+1)+1} \times \\
\frac{(2(p-k+n+1)) !}{2^{p-k+n+1}(p-k+n+1) !} .
\end{gathered}
$$

Proof of Lemma A.4. Recall that for $f: S^{1} \rightarrow \mathbb{R}$ a function that belongs to $\mathcal{B}_{1}$ and for all $u$ fixed level in $\mathbb{R}$, the coefficients $a_{f}(\cdot, u)$ are defined by $a_{f}(\boldsymbol{k}, u)=a_{f}\left(k_{1}, k_{2}\right) a\left(k_{3}, u\right)$, for $\boldsymbol{k}=\left(k_{1}, k_{2}, k_{3}\right) \in \mathbb{N}^{3}$, with

$$
a\left(k_{3}, u\right)=\frac{1}{k_{3} !} H_{k_{3}}\left(\frac{u}{\sqrt{r_{z}(0)}}\right) \phi\left(\frac{u}{\sqrt{r_{z}(0)}}\right) \frac{1}{\sqrt{r_{z}(0)}} .
$$

So for giving an expression of the coefficients $a_{f_{i}^{\star}}(\cdot, u)$ for $i=1,2$ and of $a_{\mathbf{1}}(\cdot, u)$ just give one of $a_{f_{i}^{\star}}\left(k_{1}, k_{2}\right)$ and of that of $a_{1}\left(k_{1}, k_{2}\right)$.

First, let us compute $a_{f_{1}^{\star}}\left(k_{1}, k_{2}\right)$, for $k_{1}, k_{2} \in \mathbb{N}$. Let $P:=\left(a_{i, j}\right)_{1 \leqslant i, j \leqslant 2}$. By definition of coefficient $a_{f_{1}^{\star}}\left(k_{1}, k_{2}\right)$, we have

$$
\begin{aligned}
& a_{f_{1}^{\star}}\left(k_{1}, k_{2}\right)=\frac{\sqrt{\mu}}{k_{1} ! k_{2} !} \int_{\mathbb{R}^{2}}\left(a_{11} \lambda_{1} y_{1}+a_{12} \lambda_{2} y_{2}\right) \times
\end{aligned}
$$

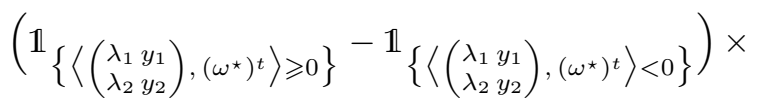

$$
\begin{aligned}
& H_{k_{1}}\left(y_{1}\right) \phi\left(y_{1}\right) H_{k_{2}}\left(y_{2}\right) \phi\left(y_{2}\right) \mathrm{d} y_{1} \mathrm{~d} y_{2} \text {. }
\end{aligned}
$$

By using the fact that $H_{n}$ is even when $n$ is even and odd if not (see (A.3) and (A.4)), the coefficients $k_{1}$ and $k_{2}$ ought to be of the same parity, otherwise the coefficients $a_{f_{1}^{\star}}\left(k_{1}, k_{2}\right)$ would be null. Therefore let us suppose first that $k_{1}=2 m$ and $k_{2}=2 \ell, m, \ell \in \mathbb{N}$. In that way and using that polynomial $H_{2 n}$ is even, one has

$$
\begin{array}{r}
a_{f_{1}^{\star}}(2 m, 2 \ell)=\frac{2 \sqrt{\mu}}{(2 m) !(2 \ell) !} \int_{\mathbb{R}^{2}}\left(a_{11} \lambda_{1} y_{1}+a_{12} \lambda_{2} y_{2}\right) \times \\
\mathbb{1}_{\left\{\left\langle\left(\begin{array}{ll}
\lambda_{1} & y_{1} \\
\lambda_{2} & y_{2}
\end{array}\right),\left(\omega^{\star}\right)^{t}\right\rangle \geqslant 0\right\}} \times H_{2 m}\left(y_{1}\right) \phi\left(y_{1}\right) H_{2 \ell}\left(y_{2}\right) \phi\left(y_{2}\right) \mathrm{d} y_{1} \mathrm{~d} y_{2} .
\end{array}
$$

Let us compute

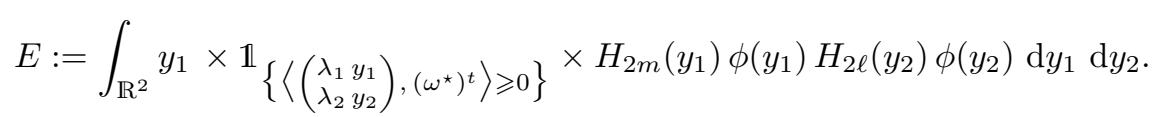

(similar computations would be done for the second integral on $y_{2}$, the arguments $\omega_{2}^{\star}, \omega_{1}^{\star}, \lambda_{2}, \lambda_{1}, \ell, m$, in this order, playing the role of $\omega_{1}^{\star}, \omega_{2}^{\star}, \lambda_{1}, \lambda_{2}, m, \ell$ in last integral). At this step of the proof Lemma A.5 is required.

Lemma A.5. Let $p \in \mathbb{N}, a \in \mathbb{R}$ and $F_{p}(a):=\int_{a}^{+\infty} z^{2 p+1} \phi(z) \mathrm{d} z$. Then

$$
F_{p}(a)=\phi(a) \sum_{k=0}^{p} \frac{2^{k} p !}{(p-k) !} a^{2(p-k)}
$$


Proof of Lemma A.5. Integration by parts and straightforward calculations give the lemma.

Three cases occur: $\omega_{1}^{\star}>0, \omega_{1}^{\star}<0$ and $\omega_{1}^{\star}=0$.

Let us consider the first one. If $\omega_{1}^{\star}>0, E$ can be written as

$$
E=\int_{\mathbb{R}} H_{2 \ell}\left(y_{2}\right) \phi\left(y_{2}\right)\left[\int_{-\frac{\lambda_{2} \omega_{2}^{\star}}{\lambda_{1} \omega_{1}^{\star}} y_{2}}^{+\infty} y_{1} H_{2 m}\left(y_{1}\right) \phi\left(y_{1}\right) \mathrm{d} y_{1}\right] \mathrm{d} y_{2} .
$$

For real $x$ and $m \in \mathbb{N}$, the polynomial form of $H_{2 m}(x)$ is

$$
H_{2 m}(x)=(2 m) ! \sum_{p=0}^{m} \frac{(-2)^{p-m}}{(2 p) !(m-p) !} x^{2 p}
$$

Using Lemma A.5, one gets

$$
\begin{array}{r}
E=(2 m) ! \sum_{p=0}^{m} \frac{(-2)^{p-m}}{(2 p) !(m-p) !} \sum_{k=0}^{p} \frac{2^{k} p !}{(p-k) !}\left(\frac{\lambda_{2} \omega_{2}^{\star}}{\lambda_{1} \omega_{1}^{\star}}\right)^{2(p-k)} \times \\
\sum_{n=0}^{\ell}(2 \ell) ! \frac{(-2)^{n-\ell}}{(2 n) !(\ell-n) !} G_{n+p-k}\left(\frac{\lambda_{2} \omega_{2}^{\star}}{\lambda_{1} \omega_{1}^{\star}}\right),
\end{array}
$$

where for $q \in \mathbb{N}$ and $x \in \mathbb{R}$, we defined

$$
G_{q}(x):=\int_{-\infty}^{+\infty} y^{2 q} \phi(y) \phi(x y) d y=\frac{1}{\sqrt{2 \pi}} \frac{1}{\left(1+x^{2}\right)^{q+\frac{1}{2}}} \frac{(2 q) !}{2^{q} q !} .
$$

If $\omega_{1}^{\star}<0$, using that for $m \in \mathbb{N}$, polynomial $H_{2 m}$ is even, one obtains that

$$
\begin{aligned}
E & =\int_{\mathbb{R}} H_{2 \ell}\left(y_{2}\right) \phi\left(y_{2}\right)\left[\int_{-\infty}^{-\frac{\lambda_{2} \omega_{2}^{\star}}{\lambda_{1} \omega_{1}^{\star}} y_{2}} y_{1} H_{2 m}\left(y_{1}\right) \phi\left(y_{1}\right) d y_{1}\right] d y_{2} \\
& =-\int_{\mathbb{R}} H_{2 \ell}\left(y_{2}\right) \phi\left(y_{2}\right)\left[\int_{-\frac{\lambda_{2} \omega_{2}^{\star}}{\lambda_{1} \omega_{1}^{\star}} y_{2}}^{+\infty} y_{1} H_{2 m}\left(y_{1}\right) \phi\left(y_{1}\right) d y_{1}\right] d y_{2},
\end{aligned}
$$

and in a same way, when $\omega_{1}^{\star}=0$,

$$
E=\left(\int_{\mathbb{R}} H_{2 \ell}\left(y_{2}\right) \phi\left(y_{2}\right) \mathbb{1}_{\left\{y_{2} \omega_{2}^{\star} \geqslant 0\right\}} \mathrm{d} y_{2}\right)\left(\int_{\mathbb{R}} y_{1} H_{2 m}\left(y_{1}\right) \phi\left(y_{1}\right) \mathrm{d} y_{1}\right)=0 .
$$

Finally, knowing that $\left|\omega_{1}^{\star}\right| \times \operatorname{sign}\left(\omega_{1}^{\star}\right)=\omega_{1}^{\star}$, one gets the expression of coefficient $a_{f_{1}^{\star}}(2 m, 2 \ell)$.

For coefficient $a_{f_{1}^{\star}}(2 m+1,2 \ell+1)$, similar arguments would be developed using the previous way and the polynomial form of $H_{2 \ell+1}(x)$, that is

$$
H_{2 \ell+1}(x)=(2 \ell+1) ! \sum_{p=0}^{\ell} \frac{(-2)^{p-\ell}}{(2 p+1) !(\ell-p) !} x^{2 p+1} .
$$

To conclude the proof of Lemma A.4, just remark that $a_{f_{2}^{\star}}(2 m+1,2 \ell)=a_{f_{2}^{\star}}(2 m, 2 \ell+1)=0$ and that $a_{f_{2}^{\star}}(2 m, 2 \ell)$ (resp. $a_{f_{2}^{\star}}(2 m+1,2 \ell+1)$ ) would be computed replacing $a_{11}$ by $a_{21}$ and $a_{12}$ by $a_{22}$ in the expression of $a_{f_{1}^{\star}}(2 m, 2 \ell)$ (resp. $a_{f_{1}^{\star}}(2 m+1,2 \ell+1)$ ). 
In order to give the coefficients $a_{1}$ we introduce the following functions. The $\beta$ function is defined by:

$$
\beta(x ; y):=\frac{\Gamma(x) \Gamma(y)}{\Gamma(x+y)}, \quad x, y>0,
$$

while the $\Gamma$ function is defined by:

$$
\Gamma(x):=\int_{0}^{\infty} e^{-t} t^{x-1} \mathrm{~d} t .
$$

Lemma A.6. The coefficients $a_{1}$ are given by: for $m, \ell \in \mathbb{N}$

$$
\begin{aligned}
& \left(a_{1}(2 m, 2 \ell) \quad a_{1}(2 m+1,2 \ell+1) \quad a_{1}(2 m, 2 \ell+1) \quad a_{1}(2 m+1,2 \ell)\right)= \\
& \left(c\left(\lambda_{1}, \lambda_{2}, m, \ell, \mu\right) \quad 0 \quad 0 \quad 0\right),
\end{aligned}
$$

where

$$
\begin{aligned}
& c\left(\lambda_{1}, \lambda_{2}, m, \ell, \mu\right):= \\
& \sqrt{2 \pi \mu} \frac{(-2)^{-(m+\ell)}}{m ! \ell !} \sum_{p=0}^{\ell} \sum_{q=0}^{m}\left(\begin{array}{c}
\ell \\
p
\end{array}\right)\left(\begin{array}{c}
m \\
q
\end{array}\right)(-1)^{p+q} \lambda_{2}\left(\frac{\lambda_{2}}{\lambda_{1}}\right)^{2 q+1} \times \\
& \sum_{n=0}^{+\infty} \frac{\left(\begin{array}{c}
q+n \\
q
\end{array}\right)\left(\begin{array}{c}
2 q+2 n \\
q+n
\end{array}\right)}{\left(\begin{array}{c}
2 q \\
q
\end{array}\right)}\left(\frac{1}{2}\right)^{2 n} \frac{1}{\beta\left(p+q+n+1 ; \frac{1}{2}\right)}\left(1-\left(\frac{\lambda_{2}}{\lambda_{1}}\right)^{2}\right)^{n} .
\end{aligned}
$$

Remark A.7. Note that in the case where $\lambda=1$, that is when the process is isotropic our result contains that of the authors expressed in Kratz and León [20, Theorem 2].

Proof of Lemma A.6. Let us compute $a_{1}\left(k_{1}, k_{2}\right)$, for $k_{1}, k_{2} \in \mathbb{N}$. We have

$$
a_{1}\left(k_{1}, k_{2}\right)=\frac{\sqrt{\mu}}{k_{1} ! k_{2}} ! \int_{\mathbb{R}^{2}} \sqrt{\lambda_{1}^{2} y_{1}^{2}+\lambda_{2}^{2} y_{2}^{2}} H_{k_{1}}\left(y_{1}\right) H_{k_{2}}\left(y_{2}\right) \phi\left(y_{1}\right) \phi\left(y_{2}\right) \mathrm{d} y_{1} \mathrm{~d} y_{2} .
$$

Similar arguments as those given in Lemma A.4 show that $a_{\mathbf{1}}\left(k_{1}, k_{2}\right)=0$ except when $k_{1}$ and $k_{2}$ are even.

So let us compute $a_{\mathbf{1}}(2 m, 2 \ell)$ for $m, \ell \in \mathbb{N}$.

The change of variable $y_{1}:=\lambda r \sin (\theta)$ and $y_{2}:=r \cos (\theta)$ in last integral and expression of $H_{2 \ell}$ given in (A.3) yield

$$
\begin{aligned}
a_{1}(2 m, 2 \ell)=\frac{2 \sqrt{\mu}}{\pi} & \frac{\lambda_{2}^{2}}{\lambda_{1}} \sum_{p=0}^{m} \sum_{q=0}^{\ell} \frac{(-2)^{p-m}(-2)^{q-\ell}}{(2 p) !(2 q) !(m-p) !(\ell-q) !} \lambda^{2 p} \times \\
& \int_{0}^{\frac{\pi}{2}} \int_{0}^{+\infty} r^{2 p+2 q+2} \cos ^{2 q}(\theta) \sin ^{2 p}(\theta) e^{-\frac{1}{2} r^{2}\left[\cos ^{2}(\theta)+\lambda^{2} \sin ^{2}(\theta)\right]} \mathrm{d} r \mathrm{~d} \theta .
\end{aligned}
$$

Now making the change of variable $r^{2}:=\frac{2 v}{\cos ^{2}(\theta)+\lambda^{2} \sin ^{2}(\theta)}$ and $w:=\sin (\theta)$, one gets

$$
\begin{array}{r}
a_{\mathbf{1}}(2 m, 2 \ell)=\frac{\sqrt{\mu}}{\pi} \frac{\lambda_{2}^{2}}{\lambda_{1}} \sum_{p=0}^{m} \sum_{q=0}^{\ell} \frac{(-2)^{p-m}(-2)^{q-\ell}}{(2 p) !(2 q) !(m-p) !(\ell-q) !} \lambda^{2 p} 2^{p+q+\frac{1}{2}} \times \\
\Gamma\left(p+q+\frac{3}{2}\right) F\left(p+q+\frac{3}{2} ; p+\frac{1}{2} ; p+q+1 ; 1-\lambda^{2}\right),
\end{array}
$$

where the hypergeometric function $F$ is defined by

$$
F(a ; b ; c ; z):=\int_{0}^{1} u^{b-1}(1-u)^{c-b-1}(1-u z)^{-a} \mathrm{~d} u .
$$

for $|z|<1,0<b<c$ and $a>0$.

The proof of lemma ensues from the following one. 
Lemma A.8. For $|z|<1,0<b<c$ and $a>0$, one has

$$
F(a ; b ; c ; z)=\frac{\Gamma(c-b)}{\Gamma(a)} \sum_{n=0}^{+\infty} \frac{\Gamma(a+n) \Gamma(b+n)}{\Gamma(c+n) \Gamma(n+1)} z^{n} .
$$

Proof of Lemma A.8. The proof consists in a serial development of function $f(z):=$ $(1-u z)^{-a}$.

Proof of Lemma 5.16. Let $f_{1}, f_{2}: S^{1} \rightarrow \mathbb{R}$ functions that belong to $\mathcal{B}_{1}$ and set $\vec{f}:=$ $\left(f_{1}, f_{2}\right)$. We have the following decomposition of $\operatorname{det}\left(\Sigma_{\vec{f}}(u)\right)$.

$$
\begin{aligned}
& \operatorname{det}\left(\Sigma_{f}(u)\right) \\
& =\Sigma_{f_{1}, f_{1}}(u) \Sigma_{f_{2}, f_{2}}(u)-\left(\Sigma_{f_{1}, f_{2}}(u)\right)^{2} \\
& =\sum_{q=1}^{+\infty} \sum_{q^{\prime}=1}^{+\infty}\left(\Sigma_{f_{1}, f_{1}}(u)\right)_{q}\left(\Sigma_{f_{2}, f_{2}}(u)\right)_{q^{\prime}}-\left(\sum_{q=1}^{+\infty}\left(\Sigma_{f_{1}, f_{2}}(u)_{q}\right)^{2}\right. \\
& =\sum_{q=1}^{+\infty} \operatorname{det}\left(\Sigma_{\vec{f}}(u)\right)_{q} \\
& +\sum_{q<q^{\prime}}\left(\sqrt{\left(\Sigma_{f_{1}, f_{1}}(u)\right)_{q}} \sqrt{\left(\Sigma_{f_{2}, f_{2}}(u)\right)_{q^{\prime}}}-\sqrt{\left(\Sigma_{f_{1}, f_{1}}(u)\right)_{q^{\prime}}} \sqrt{\left(\Sigma_{f_{2}, f_{2}}(u)\right)_{q}}\right)^{2} \\
& +2 \sum_{q<q^{\prime}}\left[\sqrt{\left(\Sigma_{f_{1}, f_{1}}(u)\right)_{q}} \sqrt{\left(\Sigma_{f_{2}, f_{2}}(u)\right)_{q^{\prime}}} \sqrt{\left(\Sigma_{f_{1}, f_{1}}(u)\right)_{q^{\prime}}} \sqrt{\left(\Sigma_{f_{2}, f_{2}}(u)\right)_{q}}\right. \\
& \left.\quad-\left(\Sigma_{f_{1}, f_{2}}(u)\right)_{q}\left(\Sigma_{f_{1}, f_{2}}(u)\right)_{q^{\prime}}\right] .
\end{aligned}
$$

To conclude the proof of lemma, we just have to verify that for all $q \in \mathbb{N}^{\star}$,

$$
\left|\left(\Sigma_{f_{1}, f_{2}}(u)\right)_{q}\right| \leqslant \sqrt{\left(\Sigma_{f_{1}, f_{1}}(u)\right)_{q}} \sqrt{\left(\Sigma_{f_{2}, f_{2}}(u)\right)_{q}} .
$$

So, let $q \in \mathbb{N}^{\star}$, and let define

$$
\left(\xi_{f}^{(n)}(u)\right)_{q}:=\frac{1}{\sqrt{\sigma_{2}\left(T_{n}\right)}} \sum_{\substack{\boldsymbol{k} \in \mathbb{N}^{3} \\|\boldsymbol{k}|=q}} a_{f}(\boldsymbol{k}, u) \int_{T_{n}} \widetilde{H}_{\boldsymbol{k}}(U(t)) \mathrm{d} t .
$$

Corollary 4.9 implies that,

$$
\left(\left(\xi_{f_{1}}^{(n)}(u)\right)_{q} ;\left(\xi_{f_{2}}^{(n)}(u)\right)_{q}\right) \underset{n \rightarrow+\infty}{\stackrel{L a w}{\longrightarrow}} \mathcal{N}\left(0 ;\left(\Sigma_{\vec{f}}(u)\right)_{q}\right),
$$

and in force $\left(\Sigma_{\vec{f}}(u)\right)_{q}$ is a semi-definite positive matrix. This argument yields inequality (A.6) and Lemma 5.16.

Proof of Lemma 5.19. Using that $B_{n}^{2}=\Sigma_{n}, \lim _{n} \Sigma_{n}=\Sigma=B^{2}$ and that $\lim _{n} \operatorname{tr}\left(B_{n}\right)=$ $\operatorname{tr}(B)>0$, we obviously deduce that $\lim _{n} B_{n}=B$. 
Estimation of local anisotropy based on level sets

\section{References}

[1] Robert J. Adler, The Geometry of Random Fields, Siam Edition, John Wiley and sons, 1981. MR0611857

[2] Robert J. Adler and Jonathan E. Taylor, Random fields and geometry, Springer monographs in mathematics, vol. 115, Springer, New York, 2007. MR2319516

[3] Denis Allard, Rachid Senoussi, and Emilio Porcu, Anisotropy models for spatial data, Mathematical Geosciences 48 (2016), no. 3, 305-328. MR3476704

[4] Ethan B. Anderes, Sourav Chatterjee, et al., Consistent estimates of deformed isotropic gaussian random fields on the plane, The Annals of Statistics 37 (2009), no. 5A, 2324-2350. MR2543694

[5] Ethan B. Anderes and Michael L. Stein, Estimating deformations of isotropic gaussian random fields on the plane, The Annals of Statistics 36 (2008), no. 2, 719-741. MR2396813

[6] Dale N. Anderson, A multivariate Linnik distribution, Statistics \& Probability Letters 14 (1992), 333-336. MR1179637

[7] Miguel A. Arcones, Limit theorems for nonlinear functionals of a stationary Gaussian sequence of vectors, The Annals of Probability 22 (1994), no. 4, 2242-2274. MR1331224

[8] Jean-Marc Azaïs and Mario Wschebor, Level sets and extrema of random processes and fields, John Wiley \& Sons, 2009. MR2478201

[9] Corinne Berzin and Ileana Iribarren, Estimation of the spectral moments matrix of second order of a gaussian random field based on the level curves, C.R. Acad. Sci. Paris, t. 307 (1988), Série I, 915-918. MR0978469

[10] Corinne Berzin, Alain Latour, and José R. León, Kac-Rice formulas for random fields and theirs applications in: random geometry, roots of random polynomials and some engineering problems, Ediciones IVIC, Instituto Venezolano de Investigaciones Científicas, Caracas, 2017.

[11] Corinne Berzin and Mario Wschebor, Approximation du temps local des surfaces gaussiennes, Probability Theory and Related Fields 96 (1993), 1-32. MR1222362

[12] Enrique M. Cabaña, Affine processes: a test of isotropy based on level sets, SIAM Journal on Applied Mathematics 47 (1987), no. 4, 886-891. MR0898839

[13] Eugene B. Dynkin, Self-intersection gauge for random walks and for brownian motion, The Annals of Probability 16 (1988), 1-57. MR0920254

[14] Anne Estrade and José R. León, A central limit theorem for the Euler characteristic of a Gaussian excursion set, Ann. Probab. 44 (2016), no. 6, 3849-3878. MR3572325

[15] Herbert Federer, Geometric measure theory, Springer, 1969. MR0257325

[16] Francky Fouedjio, Nicolas Desassis, and Thomas Romary, Estimation of space deformation model for non-stationary random functions, spatial statistics 13 (2015), 45-61. MR3368820

[17] Julie Fournier. Identification and isotropy characterization of deformed random fields through excursion sets. Adv. in Appl. Probab., 50 (2018), no. 3, 706-725. MR3877250

[18] Izrail S. Gradshteyn and Iosif M. Ryzhik, Table of Integrals, Series, and Products, Academic Press, Seventh Edition, 2007. MR0669666

[19] Ileana Iribarren, Asymptotic behaviour of the integral of a function on the level set of a mixing random field, Probability and Mathematical Statistics, 10 (1989), Fasc. 1, 45-56. MR0990398

[20] Marie F. Kratz and José R. León, Central limit theorems for level functionals of stationary gaussian processes and fields, Journal of Theoretical Probability 14 (2001), no. 3, 639-672. MR1860517

[21] S.C. Lim and Lee P. Teo, Gaussian fields and Gaussian sheets with generalized Cauchy covariance structure, Stochastic Processes and their Applications 119 (2009), 1325-1356. MR2508576

[22] S.C. Lim and Lee P. Teo, Generalized Whittle-Matérn field as a model of correlated fluctuations J. Phys. A: Math. Theor. 42 (2009), 1-20. MR2485858

[23] Péter Major, Multiple wiener-itô integrals: With applications to limit theorems (volume 849 of lecture notes in mathematics), 1981. MR0611334 
Estimation of local anisotropy based on level sets

[24] Ivan Nourdin, Giovanni Peccati, et al., Stein's method and exact berry-esseen asymptotics for functionals of gaussian fields, The Annals of Probability 37 (2009), no. 6, 2231-2261. MR2573557

[25] David Nualart, Giovanni Peccati, et al., Central limit theorems for sequences of multiple stochastic integrals, The Annals of Probability 33 (2005), no. 1, 177-193. MR2118863

[26] Giovanni Peccati and Ciprian A. Tudor, Gaussian limits for vector-valued multiple stochastic integrals, Séminaire de Probabilités XXXVIII 1857 (2005), 247-262. MR2126978

[27] Olivier Perrin and Rachid Senoussi, Reducing non-stationary random fields to stationarity and isotropy using a space deformation, Statistics \& probability letters 48 (2000), no. 1, 23-32. MR1767607

[28] Stephen O. Rice, Mathematical analysis of random noise, Bell Labs Technical Journal 23 (1944), no. 3, 282-332. MR0010932

[29] Paul D. Sampson and Peter Guttorp, Nonparametric estimation of nonstationary spatial covariance structure, Journal of the American Statistical Association 87 (1992), no. 417, 108-119.

[30] Eric V. Slud, Mwi representation of the number of curve-crossings by a differentiable gaussian process, with applications, The Annals of probability 22 (1994), 1355-1380. MR1303648

[31] Zachary D. Weller, Jennifer A. Hoeting, et al., A review of nonparametric hypothesis tests of isotropy properties in spatial data, Statistical Science 31 (2016), no. 3, 305-324. MR3552737

[32] Mario Wschebor, Surfaces aléatoires: mesure géométrique des ensembles de niveau, vol. 1147, Springer, 1985. MR0871689

[33] Akiva M. Yaglom, Correlation Theory of Stationary and Related Random Functions, Volume I: Basic Results, Springer Series in Statistics, Springer-Verlag New York, 1987. MR0893393

[34] Akiva M. Yaglom, Correlation Theory of Stationary and Related Random Functions, Volume II: Supplementary Notes and References, Springer Series in Statistics, Springer-Verlag New York, 1987. MR0915557

Acknowledgments. The author is grateful to the referees for many suggestions that led to substantial improvements in this work. 